\title{
Neuronal Activation of the Gastrointestinal Tract Shapes the Gut Environment in Mice
}

\author{
Bryan B. Yoo ${ }^{1 *}$, Jessica A. Griffiths ${ }^{1}$, Peter Thuy-Boun ${ }^{2+}$, Victor Cantu ${ }^{3+}$, Kelly \\ Weldon $^{4,5,+}$, Collin Challis ${ }^{1}$, Michael J. Sweredoski ${ }^{1}$, Ken Y. Chan ${ }^{1 \#}$, Taren M. \\ Thron ${ }^{1}$, Gil Sharon ${ }^{1}$, Annie Moradian ${ }^{1 \%}$, Gregory Humphrey ${ }^{3}$, Qiyun Zhu ${ }^{3}$, Justin \\ Shaffer ${ }^{3}$, Dennis W. Wolan ${ }^{2}$, Pieter C. Dorrestein ${ }^{3,4,5}$, Rob Knight ${ }^{3,5,6,7}$, Viviana \\ Gradinaru', and Sarkis K. Mazmanian $^{1 *}$ \\ ${ }^{1}$ Division of Biology \& Biological Engineering, California Institute of Technology, Pasadena, California, \\ USA, 91125 \\ ${ }^{2}$ Departments of Molecular Medicine and Integrative Structural and Computational Biology, The Scripps \\ Research Institute, 10550 North Torrey Pines Road, La Jolla, CA 92037, USA \\ ${ }^{3}$ Department of Pediatrics, University of California San Diego, San Diego, CA, USA \\ ${ }^{4}$ Collaborative Mass Spectrometry Innovation Center, Skaggs School of Pharmacy and Pharmaceutical \\ Sciences, University of California San Diego, San Diego, CA, USA \\ ${ }^{5}$ UCSD Center for Microbiome Innovation, University of California San Diego, San Diego, CA, USA \\ ${ }^{6}$ Department of Computer Science and Engineering, University of California San Diego, San Diego, CA, \\ USA \\ ${ }^{7}$ Department of Engineering, University of California San Diego, San Diego, CA, USA \\ +These authors contributed equally \\ \#Present Address: Stanley Center for Psychiatric Research, Broad Institute, Massachusetts Institute of \\ Technology, Cambridge, MA, 02142, USA \\ \% Present Address: Precision Biomarker Labs, Cedars-Sinai, 8700 Beverly Blvd. Davis 2904, Los \\ Angeles, CA 90048
}

*Corresponding Author. Email: byoo@caltech.edu (B.B.Y); sarkis@caltech.edu (S.K.M) 


\begin{abstract}
The enteric nervous system (ENS) coordinates responses that shape the intestinal milieu, and participates in bi-directional communication with various organs including the brain. Tools to study, and knowledge of, ENS development and function lag behind brain research. Herein, we deploy recombinant adeno-associated viral (rAAV) vectors with enhanced tropism for the gut to map and activate gut associated neurons in mice with spatial and temporal resolution. $\mathrm{rAAV}$-mediated fluorescent labelling coupled with whole-tissue clearing methods in the small intestine and colon reveal novel neuronal architectures in the gut of mice. Viral delivery of genetically-encoded calcium indicators enable in vivo measurement of ENS activity. Further, we employed chemogentics to specifically activate gut neurons that express choline acetyltransferase $\left(\mathrm{ChAT}^{+}\right)$or tyrosine hydroxylase $\left(\mathrm{TH}^{+}\right)$. Targeted activation of $\mathrm{ChAT}^{+}$or $\mathrm{TH}^{+}$ neuronal populations associated with the gastrointestinal (Gl) tract alters the intestinal transcriptome, mouse and microbial proteomes, metagenome, and fecal metabolome. We reveal previously unknown and broad roles for enteric neurons in modulating Gl physiology, mucosal immunity, and microbiome structure, and provide evidence for novel functions by the ENS such as regulating fungal colonization and shaping of bile acid profiles in the gut. These advanced experimental tools and rich datasets with multi-parameter characterization of the gut ecosystem may facilitate expanded understanding and study of the ENS.
\end{abstract}




\section{Introduction}

The gastrointestinal (Gl) tract consists of diverse cells (e.g., immune, epithelial, endothelial, endocrine, muscle, neuronal, and even microbial) that coordinate physiology within the gut (Furness, 2006) and throughout the body (Rao and Gershon, 2016). The mammalian gut receives and transmits neuronal signals from over 100,000 extrinsic nerve fibers that originate from the sympathetic and parasympathetic nervous systems (Grundy and Brookes, 2011). The GI tract is also innervated by an expansive network of intrinsic neurons, collectively termed the enteric nervous system (ENS), that has over 100 million neurons organized into distinct compartments within the Gl tract, namely the myenteric plexus and submucosal plexus (Furness et al., 2014). In recent years, extrinsic and intrinsic innervation of the GI tract has been implicated in various host responses, from digestion (Schneider et al., 2019), to immunity (Gabanyi et al., 2016; Muller et al., 2014), to complex behaviors (Bravo et al., 2011) in mice. Specific interactions between neurons of the GI tract and various other cell types highlight the diverse and expansive roles of the ENS. For example, neural pathways in the gut regulate nutrient sensation through intestinal enteroendocrine cells (Kaelberer et al., 2018), affect thesepithelial barrier and mucosal immunity (Jarret et al., 2020; Seillet et al., 2020; Talbot et al., 2020), and dynamically interface with the microbiome (Lai et al., 2019; Matheis et al., 2020). While neurons in the central nervous system (CNS) interact with multiple cell types, exposure of the ENS to changing external inputs such as from diet, microbiome, xenobiotics, etc. create interactions distinct from those in the brain.

With the goal of exploring complex interactions in the Gl tract, we employed and optimized powerful experimental tools, originally developed for brain research, to map the architecture of the ENS in mice and the functional consequence of neuronal activation of the Gl tract. Adenoassociated viruses (AAVs) have been characterized for their ability to efficiently deliver various genes to eukaryotic cells (Kotterman and Schaffer, 2014), and have been used in research, biotechnology, and medical settings (Samulski and Muzyczka, 2014). Employing an engineered AAV capsid variant, AAV-PHP.S, that was selected for having enhanced tropism within the ENS and other peripheral ganglia of mice (Chan et al., 2017), we achieved broad expression of genetically encoded fluorescent reporters and neural activators in the gut. Further, we delivered a fluorescent calcium reporter (GCaMP6F) into enteric neurons to measure ENS activity in live animals. Adding to current knowledge gained through immunohistochemical characterization of the ENS (Furness, 2012), we coupled tissue clearing technologies with AAV-mediated labelling 
of cells to present methodological improvements to traditional approaches that allowed broad surveyance of ENS architectures within intact GI tissue and the ability to directly measure in vivo neuronal activity in the gut.

Beyond characterization, we sought to investigate the effects of activating specific neuronal subsets on the gut environment. Choline acetyltransferase (ChAT) and tyrosine hydroxylase $(\mathrm{TH})$ are the rate limiting enzymes in acetylcholine and catecholamine biosynthesis, respectively, and are key chemical mediators of neurotransmission in the brain and the periphery. However, the extent to which these neuronal populations impact complex GI physiologies beyond motility and secretory function remain less studied. Accordingly, we transduced GI neurons of mice with chemogenetic modulators (Wess et al., 2013) in discrete cell populations to achieve cell-specific control of ChAT and TH neurons. We discovered that stimulating neural activity in the GI tract alters the transcriptional and proteomic landscape of the intestines, with complementing alterations to the gut metagenome and metabolome. We report diverse and expansive effects of directed neuronal activation on epithelial biology, mucosal immunity, angiogenesis, and muscle physiology, among other outcomes. We observe expansions and contractions of fungal and bacterial taxa. Of the many molecular changes, we highlight that ENS activation has dramatic effects on the conversion of primary to secondary bile acids, products of mouse and bacterial co-metabolism that have been widely implicated in health and disease (de Aguiar Vallim et al., 2013; Fiorucci et al., 2018; Hofmann and Eckmann, 2006; Long et al., 2017; Sakai et al., 1980). The enhanced experimental tools and approaches, and multi-parameter datasets, introduced in this Tools and Resource may help advance technologies and understanding of neurogastroenterology and the complex environment of the gut in mice.

\section{Results}

Targeted neuronal labeling enables extensive, high-resolution imaging of the mouse ENS Various morphologic features of the ENS have been characterized (Furness, 2006). However, resolving the broad architecture of neurons in the gut with current imaging methods, primarily using immunohistochemistry ( $\mathrm{IHC})$, are limited by several physical obstacles of the $\mathrm{Gl}$ tract: large area ( $40 \mathrm{~cm}$ long in a mouse), gut motility / tissue pliability (i.e., with or without luminal contents, relaxed vs. contracted smooth muscle), and 3-dimensional biogeography (i.e., longitudinal and cross-sectional analysis). Furthermore, the ENS is localized within two distinct 
plexuses of the Gl tract and studies have often characterized myenteric neurons by microdissection of the longitudinal muscle / myenteric plexus tissue layer (Fried and Gulbransen, 2015), a manipulation of the tissue that disassociates axons from their destination in 3-dimensional space and overlooks the organization of submucosal neurons.

To overcome these limitations, we labelled enteric neurons in living mice with recombinant adeno-associated viruses ( $\mathrm{AAAVs}$ ) expressing fluorescent proteins, and used tissue clearing techniques to enhance visualization of intact Gl tissue (https://github.com/mazmanianlab/Yoo_et_al_2021/tree/main/ENS\%20quantification). The AAV capsid variant AAV-PHP.S was used since it has been optimized for systemic delivery in mice (Deverman et al., 2016) and characterized for its increased tropism to the peripheral nervous system, including the ENS (Chan et al., 2017). We first looked to compare the general infectivity of AAV-PHP.S to the traditionally-used AAV9, which has been empolyed in the past for viral delivery to the ENS (Benskey et al., 2015). Similar to Chan et al., the cargo for both AAVs was the gene encoding a nuclear localized green fluorescent protein expressed under the strong, ubiquitous CAG promoter. Following a single intravenous (i.v.) systemic injection of the AAVs, AAV-PHP.S efficiently transduced cells throughout the Gl tract and exhibited greater infectivity of cells throughout layers of the GI tract, compared to AAV9, as seen by increased cellular expression of nuclear localized CAG-GFP (Figure 1A). Gene products driven by the human Synapsin 1 (hSYN1) promoter have previously been shown to restrict expression to neurons (Jackson et al., 2016; Kügler et al., 2003) and minimize expression in peripheral targets such as the dorsal root ganglia (Haenraets et al., 2017). We packaged fluorescent proteins genes under the hSYN1 promoter into AAV-PHP.S, delivered them systemically, and found that $~ 90 \%$ $( \pm 1.5 \%)$ of cells in the ENS labelled with antibodies against $\underline{P}$ rotein Gene Product 9.5 (PGP9.5), a pan-neuronal protein, co-localized with virally labelled neurons in the small intestines (SI) and colon (Figure 1B).

Efforts to study the circuitry and neurochemistry of the gut nervous system have been hampered by the physical length of the GI tract, making broad characterization of the ENS by IHC both technically challenging and costly. With AAV-mediated labeling, a single systemic injection of AAV-PHP.S:hSYN1-mNeonGreen at $10^{12}$ viral genomes (vg) was sufficient to label expansive regions of ENS as seen in the SI and colon Figures 1C, Figure 1 - Figure Supplement $1 \mathrm{~A}$ and $1 \mathrm{~B}$ ). This minimizes the technical variability inherent to $\mathrm{IHC}$ staining of numerous tissue sections, and enables uniform imaging of millimeters of tissue at micron 
resolution across many samples. Pan tissue labeling revealed spatial diversity of the ENS, such as architectural differences of ganglia proximal and distal to the mesentery (insets in Figure 1C), helping orient images relative to each other and thus facilitating 3-dimensional tissue reconstruction. Indeed, coupling viral-labeling with tissue clearing methods, such as a sorbitolbased clearing method (ScaleS) (Hama et al., 2015; Treweek et al., 2015; Yang et al., 2014), we were able to preserve the structure of intestinal tissue. Imaging intact tissue enabled a global view of the ENS including extrinsic nerve processes in the gut (Figure 1 - Figure Supplement $1 \mathrm{~A}$ and 1B), how ENS architecture changes in association with Peyer's patches (small intestinal lymphoid tissues) (Figure 1 - Figure Supplement 1C and 1D), and revealed different patterns of ENS ganglionic morphology when tissue is fixed with or without luminal contents (Figure 1 Figure Supplement 1E-1G).

\section{Multicolor labeling to trace axons across vast tissue distances}

When three separate viruses, each encoding a distinct fluorescent protein (PHP.S:hSYN1mTurquoise2, PHP.S:hSYN1-mNeonGreen, or PHP.S:hSYN1-mRuby2 [together AAVPHP.S:hSYN1-XFP]), are delivered together at a combined dose of $10^{12} \mathrm{vg}$, stochastic and combinatorial viral transduction of cells resulted in multi-color labelling of neurons. Labeling of neighboring cells with different colors demarcated cellular boundaries (Figure 1D) and morphologies (Figure 1E) much more clearly than IHC, and allowed unambiguous assignment of axons to specific cell bodies from which they emanate. This approach is also tunable by the design of genetic promoters for different cell types, for example labeling of enteric glial cell bodies with virally-expressed tdTomato driven by the glial fibrillary acidic protein (GFAP) promoter (Figure 1F).

Lastly, passive CLARITY (PACT) tissue clearing (Hama et al., 2015; Treweek et al., 2015; Yang et al., 2014) facilitated imaging of both myenteric and submucosal neurons within the same image stack captured by confocal microscopy (Figure 1G). This method allows direct imaging of the ENS in intact GI tissue via whole mount microscopy, eliminating the need to microdissect distinct layers of the Gl tract and prepare thin sections that full tissue reconstruction challenging, and also reduces mechanically-introduced artifacts to tissue. Together, this cell labelling approach provides an effective and economical method to label the ENS at high resolution, with cell specificity, with spatial positioning throughout the tissue, and is widely deployable without the need for genetically-engineered mouse strains. While we demonstrate the cellular versatility of this method, the remainder of this study focuses on investigating neurons in the gut. 


\section{Characterizing and quantifying ENS architecture}

The small intestine and colon display similar, divergent, and synergistic functions (Hansen, 2003). However, it remains poorly understood how the cellular architecture of the ENS contributes to Gl function, and broad, simultaneous characterization of the myenteric and submucosal plexuses has remained elusive (Foong et al., 2014; Sang and Young, 1998; Steele et al., 1991). Wild-type mice were transduced with AAV-PHP.S:hSYN1-XFP via a single i.v. injection to broadly label enteric neurons in the gut (Figure 2A). Viral infection was uniform throughout the SI and colon (Figure 2 - Figure Supplement $2 \mathrm{~A}$ ), outside of a small $\sim 1.5 \mathrm{~cm}$ section of the medial colon that, for unknown reasons, was consistently not well transduced and was therefore excluded from further analysis. Seven regions of the SI and two regions of the colon were sampled proximally to distally. Tissue regions were approximately $1 \mathrm{~cm}$ in length and sampled every 2 to $3 \mathrm{~cm}$. General ENS architecture was explored by quantifying the number of neurons and ganglia, as well as the size of ganglia (i.e., the number of neurons in each ganglion) in the myenteric and submucosal plexuses (Figures 2B-2G), normalized to the number of crypts to control for inherent variabilities within tissues. Importantly, no differences were found in crypt diameter along the Gl tract (Figure 2 - Figure Supplement 2B). We saw that in the SI, the number of neurons and ganglia generally increased toward the distal portions of the myenteric plexus compared to proximal regions, while the converse was true for neurons and ganglia in the submucosal plexus (i.e., lower numbers in distal relative to proximal regions) (Figures 2B and 2C). Additionally, the size of the ganglia (i.e., the number of neurons per ganglia) increased in the distal regions of the SI myenteric plexus, a feature not observed in the submucosal plexus (Figure 2D). While neuronal numbers were similar in the proximal and distal regions of the colonic neural plexuses (Figure $2 \mathrm{E}$ ), the number of myenteric ganglia increased (Figure 2F) while the size of each ganglion decreased in the distal colon (Figure 2G). Interestingly, submucosal neurons in the proximal colon were localized to the natural folds the tissue (Figure 2A, dashed lines in lower right panel), and these ganglia appear smaller in the distal colon. We conclude that combining viral labelling with tissue clearing enables exceptionally broad analysis of both myenteric and submucosal neurons throughout the gut, uncovering biogeographic features of ENS architecture in mice.

\section{Measuring neuronal activity in the ENS}

Existing approaches to measure intracellular calcium flux, a surrogate for neuronal firing, in the ENS include ex vivo preparations of intestinal tissue (Hennig et al., 2015) or surgically 
implanting an abdominal window to obtain in vivo ENS calcium recordings (Rakhilin et al., 2016; 2019). These techniques involve extensive manipulation of tissue to obtain access to the longitudinal muscle and myenteric plexus layer, require physical separation of the ENS from its extrinsic neural connectivity, and are technically challenging. We sought to develop a more tractable method to directly assess the activity of specific neurons in the ENS of live mice.

A genetically-encoded calcium indicator, GCaMP6F (Chen et al., 2013), driven by the CAG promoter was targeted to the ENS via systemic viral delivery with AAV-PHP.S. Mice were anesthetized, intestines removed from the peritoneum, and unstimulated calcium transients in the proximal colon were recorded in individual neurons by in vivo confocal microscopy (Figure $2 \mathrm{H}$ and Video Supplement 1). Neurons in myenteric ganglia were determined by their size and those that displayed robust changes in fluorescence intensity were chosen at random for analysis. Measuring neuronal calcium flux over time revealed an average spike rate of 29.1 spikes/minute with an interquartile range of 19.0 - 37.7 spikes $/$ minute $(\mathrm{min} .=7.0$ spikes $/ \mathrm{min}$., max.=63.0 spikes/min) (Figure 2l). Time between calcium spikes was calculated for each cell, and the average peak interval was 2.6 seconds with an interquartile range of 1.6 - 3.2 seconds ( $\min .=1.0$ seconds, max.=8.6 seconds) (Figure $2 \mathrm{~J}$ ). Heatmaps (Figure $2 \mathrm{~K}$ ) and traces (Figure 2L) constructed from GCaMP6F fluorescence intensities further display the heterogeneity of neuronal firing among and within cells (i.e., tonic vs. phasic, small vs. large fluorescence delta, duration of spike). These data highlight the heterogeneity of ENS activity and underscores the resolution of our method in achieving cell specific measurements of neuronal activity (https://github.com/mazmanianlab/Yoo_et_al_2021/tree/main/gcamp6f).

\section{Mapping neuronal subsets in the ENS}

Subpopulations of neurons can be defined by the neurotransmitters they produce, and are often demarcated by the rate limiting enzymes required for neurotransmitter production. Choline acetyltransferase (ChAT) and tyrosine hydroxylase $(\mathrm{TH})$ are the rate limiting enzymes in acetylcholine and catecholamine biosynthesis, respectively. In the GI tract, acetylcholine is a major excitatory neurotransmitter mainly studied for its role in motility and secretomotor function (Furness, 2012; Mittal et al., 2017). Although $\mathrm{ChAT}^{+}$and $\mathrm{TH}^{+}$neurons are intrinsic to the Gl tract, they have yet to be systematically characterized and interrogated for their role(s) in GI physiology. We employed ChAT-Cre and TH-Cre animals, where the Cre-recombinase (Cre) is expressed under the control of each respective gene promoter. Technically, genetic constructs can be engineered to have the payload transgene in a double-floxed inverse orientation (DIO), 
such that the transgene is flipped and expressed (and protein translated) in a Cre-dependent manner; in other words, AAV's deliver transgenes that are expressed in ChAT+ or TH+ neurons only.

Accordingly, we transduced ChAT-Cre or TH-Cre mice with AAV-PHP.S:hSYN1-DIO-XFP and cleared tissues using ScaleS to visualize these neuronal subpopulations. Remarkably, each neuronal population occupied spatially distinct layers of the Gl tract, with ChAT ${ }^{+}$neurons primarily localized to the myenteric plexus whereas $\mathrm{TH}^{+}$neurons were more abundant in the submucosal plexus (Figure 3A). Upon quantification, there are more $\mathrm{ChAT}^{+}$than $\mathrm{TH}^{+}$neurons in all assayed regions of the SI (Figure 3 - Figure Supplement $3 \mathrm{~A}$ ), an increase largely due to higher cell numbers in the myenteric plexus (Figure 3B). However, there were more $\mathrm{TH}^{+}$ submucosal neurons in the small intestine compared to $\mathrm{ChAT}^{+}$cells (Figure $3 \mathrm{C}$ ), and this spatial disparity was accentuated when calculating the ratio of myenteric-to-submucosal neurons (Figure 3 - Figure Supplement 3B). Furthermore, densities of myenteric neurons increased distally in the SI of TH-Cre mice (Figure 3B, SI-1 vs SI-10/13/15, p < 0.01; SI-4 vs SI-10/13/15 p $<0.05)$, though still lower than neuronal numbers in ChAT-Cre mice. In the colon, the ratio of myenteric and submucosal neurons did not differ between $\mathrm{ChAT}^{+}$and $\mathrm{TH}^{+}$cells (Figure 3 Figure Supplement 3B). These data reveal that spatial localization of $\mathrm{ChAT}^{+}$and $\mathrm{TH}^{+}$neurons is distinct. Additional subsets of neurons in the gut can be mapped by this approach.

\section{Activation of $\mathrm{ChAT}^{+}$and $\mathrm{TH}^{+}$neurons alters the intestinal transcriptome}

The unique spatial organization of $\mathrm{ChAT}^{+}$and $\mathrm{TH}^{+}$neurons suggests potentially distinct functions that can be investigated through cell-specific activation of each neuronal subpopulation. ChAT-Cre or TH-Cre mice were infected with Cre-dependent genetic constructs encoding activating 'Designer $\underline{R}$ eceptors $\underline{E} x c l u s i v e l y \underline{A}$ ctivated by $\underline{D}$ esigner $\underline{D}$ rugs' or DREADDs (AAV-PHP.S:hSYN1-DIO-hM3Dq-mRuby2). hM3Dq is a modified neurotransmitter receptor that is designed to induce neuronal activation when exposed to Compound 21 (C21), a "designer drug" that activates only this receptor (Thompson et al., 2018). Accordingly, C21 induces a calcium transient in a Cre-expressing neuron activated by DREADDs, as shown by the gradual increase in GCaMP6F fluorescence in an intestinal explant (Figure 3D, Video Supplement 2), validating the approach.

ChAT-Cre or TH-Cre mice were infected with activating DREADDs or a control virus expressing a reporter protein instead (AAV-PHP.S:hSYN1-DIO-mRuby2). C21 was administered 
intraperitoneally (i.p.), once daily for 10 consecutive days to all mice (Figure 3E). One hour following the last $\mathrm{C} 21$ injection, $1 \mathrm{~cm}$ of tissue from the distal SI and proximal colon was harvested, and gene expression profiled by QuantSeq, a quantitative 3' mRNA-sequencing technology (https://github.com/mazmanianlab/Yoo_et_al_2021/tree/main/RNAseq). In neurons, the rapid and transient expression of immediate early genes (IEGs) is widely used as a measure of increased neural activity (Wu et al., 2017). Accordingly, the IEGs Fos, Egr1, Jun, Klf2 (Figures 3F-3I) were among the most significantly upregulated transcripts in the SI and colon of both ChAT-Cre and TH-Cre mice harboring the activating DREADD (Figure 3J). Moreover, IEGs identified here are known to be upregulated during growth and differentiation of highly active cell types such as immune cells (Bahrami and Drabløs, 2016; Ramirez-Carrozzi et al., 2009), smooth muscle cells (Miano et al., 1993), and intestinal epithelial cells (Flandez et al., 2008).

In the distal SI, DREADD activation produced similar numbers of differentially expressed genes (DEGs; $\left.p_{a d j .}<0.05\right)$ in ChAT-Cre mice (162 DEGs) (Figure 3F) and TH-Cre mice (165 DEGs) (Figure $3 \mathrm{H}$ ). Interestingly however, $\sim 73 \%$ of the DEGs were upregulated upon ChAT-Cre activation (118 up, 44 down) and only $\sim 42 \%$ of the DEGs were upregulated upon TH-Cre activation (69 up, 96 down). This parallels the number of upregulated IEGs in the distal SI of ChAT-Cre mice (29 IEGs) compared to those in TH-Cre mice (2 IEGs) (Figure 3J), with a few IEGs (i.e., Hbegf, Soca3, Mcl1) being downregulated in TH-Cre mice (Figure 3H). Similar proportions of transcripts were upregulated in the proximal colon of both ChAT-Cre (253 DEGs169 up, 84 down) (Figure 3G) and TH-Cre mice (192 DEGs - 130 up, 62 down) (Figure 3I).

Gene Set Enrichment Analysis (GSEA) was employed to identify differences in gene ontology (GO) annotations between treatment groups (Figures 3K-N and Figure 3 - Table Supplement 1), providing insight into cellular function. Notably, the most highly enriched GO term in the distal SI of ChAT-Cre mice was "regulation of smooth muscle cell proliferation" (Figure 3K), whereas in TH-Cre mice it was the "response to bacteria" (Figure 3M). In the proximal colon, we observed more similarity in GO pathways between ChAT-Cre and TH-Cre mice following DREADDmediated activation (Figures $3 \mathrm{~L}$ and $3 \mathrm{~N}$ ). These gene expression profiles support the implication that spatial localization may drive distinct transcriptomic signatures depending on the neurons activated (see Figures 3A-3C), i.e., ChAT ${ }^{+}$neurons in the myenteric plexus of the SI are adjacent to muscle cells, while $\mathrm{TH}^{+}$neurons in the distal $\mathrm{SI}$ are enriched in the submucosal plexus that neighbor epithelial and immune cells which respond to bacteria. Both neuronal subsets are abundant in the myenteric plexus of the colon (see Figure $3 \mathrm{~B}$ ). Also, the pathways 
upregulated where neurons exist in both myenteric and submucosal plexuses portray a wider breadth of changes than activation of $\mathrm{TH}^{+}$neurons, with $\mathrm{ChAT}^{+}$neuronal activation impacting diverse cellular functions that take place in the Gl tract, involving endothelial, epithelial, immune, and adipose cells (Figure 3L and Figure 3 - Table Supplement 1). We do not know if these gene expression profiles are a direct or indirect result of neuronal activation, as changes in the intestinal milieu (as described below) may feedback on cells to alter their transcriptome. While recent studies have employed single-cell RNAseq technologies to profile transcriptomes of ENS neurons (Drokhlyansky et al., 2020), the power to selectively activate specific enteric neurons will open new avenues to explore the dynamic interplay between cells of various lineages in the gut.

\section{Neural subsets differentially impact the luminal gut proteome}

Proteins from the mouse, gut microbes, and diet converge and interact in the Gl tract (Albenberg and $\mathrm{Wu}, 2014$ ). To determine effects of specific neuronal subset activation on the gut environment, we performed untargeted label-free proteomics on cell-free supernatants of cecal contents from ChAT-Cre and TH-Cre mice, with and without neuronal activation by DREADDs (https://github.com/mazmanianlab/Yoo_et_al_2021/tree/main/proteomics). Material from the cecum (a compartment at the juncture of the SI and colon in mice) were collected one hour following the final C21 treatment (see Figure 3E), and analyzed by liquid chromatography / tandem mass spectrometry (LC-MS / MS). Interestingly, the abundance of Niemann-Pick C1Like 1 protein (NPCL1) is increased in the cecum of activated ChAT-Cre mice. NPCL1 is expressed on the apical surface of enterocytes, and is an integral protein involved in the absorption of free cholesterol from the lumen, the precursor of bile acids (Jia et al., 2011). Goblet-cell related proteins also trended upwards, specifically Mucin-19 (MUC19) and Zymogen granule 16 (ZG16) (Figure 4A), a protein localized to secretory granules (Rodríguez-Piñeiro et al., 2013). Conversely, one of the most highly downregulated proteins was an aldehyde dehydrogenase (Q3U367) encoded by the Aldh9A1 gene, which is involved in the catalytic conversion of putrescine to gamma-aminobutyric acid (GABA) (Matsushima et al., 1986). Little is known about the role of GABA in the ENS. Pancreatic digestive enzymes were the most significantly upregulated proteins in cecal contents of ChAT-activated mice (Figure 4A). These include chymopasin (CTRL), chymotrypsinogen 1 (CTRB1), and pancreatic lipase related protein 2 (PNLIPRP2). Accordingly, network analysis of upregulated proteins revealed that KEGG pathways associated with digestion represent the majority of the network (Figure 4B). This novel finding is consistent with evidence that cholinergic, viscerofugal neurons send signals 
from the Gl tract to other organs of the digestive system, including the pancreas (Furness et al., 2014).

Activation of $\mathrm{TH}^{+}$neurons also altered the luminal proteome of the cecum. Interestingly, $88 \%$ $(52 / 59)$ of the differentially abundant proteins $\left(p_{\text {adj. }}<0.25, p_{n o m<} 0.021\right)$ are distinct from those identified in activated ChAT-Cre mice $\left(p_{\text {adj. }}<0.25, p_{n o m}<0.042\right)$. Also $90 \%$ of the cecal proteins in TH-Cre mice were upregulated (53/59) compared to only $18 \%$ of proteins in ChAT-Cre mice (20/112), suggesting that activation of distinct neuronal subsets is associated with largely distinct changes in Gl function. We observed signatures of increased protein-protein interactions in cecal contents of activated TH-Cre mice, evidenced by more network nodes and connections (Figures $4 \mathrm{C}$ and 4D). When $\mathrm{TH}^{+}$neurons are activated, Filamin $\mathrm{B}$ (FLNB) and spectrin beta chain, non-erythrocytic 1 (SPTB2) were two of the most significantly enriched proteins (Figure $4 \mathrm{C}$ ), and both are associated with the intestinal brush border and membrane vesicles (Donowitz et al., 2007; McConnell et al., 2011). Accordingly, vesicle-mediated transport was a major protein network we identified (Figure 4D) and relatedly, coatomer proteins also trended upward (COPA and COPB2) (Figure 4C). Other enriched protein interaction networks that emerged were associated with the immune system, metabolic pathways, and ribosomes (Figure 4D). For example, immune related proteins such as immunoglobulin A ( $\lg A)$ (in ChAT-Cre) and $\lg$ G2b and complement component $\mathrm{C} 3$ (in TH-Cre) trended upward (Figure 4A and 4C), results that are in accord with the immune-related biological processes identified by gene expression after neuronal activation (see Figure 3).

Perhaps the most intriguing observation was the strong depletion of acidic mammalian chitinase (CHIA) upon activation of $\mathrm{TH}^{+}$neurons (Figure 4C). Chitin is a natural polysaccharide that comprises a major component of fungal cell walls (Latgé, 2007). Intestinal chitinases are poorly studied in mice and CHIA has not been investigated for its role in regulating fungal populations in the gut. Because our samples contain host and microbial proteins, this result prompted us to search our dataset against microbial protein databases. Remarkably, the decrease in CHIA abundance following $\mathrm{TH}^{+}$neuron activation was accompanied by a large bloom in the proportion of fungal-associated peptides in the microbiome (representing $~ 59 \%$ of peptides mapped to any microbe) (Figure 4E). In contrast, fungal peptides represented only $\sim 0.4 \%$ of enriched peptides in the lumen of activated ChAT-Cre mice (Figure 4F). We propose that reduction of chitinase production by activated $\mathrm{TH}^{+}$cells is directly associated with the observed dramatic increase in fungal proteins, and if true, would represent a novel circuit by which mice regulate fungal loads 
in the gut. Taken together, profiling the gut lumenal proteome upon neuronal activation revealed intriguing changes, with $\mathrm{ChAT}^{+}$neurons preferentially impacting digestion and $\mathrm{TH}^{+}$neurons preferentially involved in host-microbe interactions.

\section{Activation of ENS neurons reshapes the gut microbiome}

Given the observed changes in the gut proteome, including proteins of microbial origin, we reasoned that neuronal activation in the ENS may impact the composition and community structure of the gut microbiota. Accordingly, the microbiome was profiled via shotgun metagenomics from a longitudinal collection of fecal samples prior to and following $\mathrm{ChAT}^{+}$or $\mathrm{TH}^{+}$neuron activation (pre-C21 samples, and at days 1, 5, and 9 after initial C21 dose) and cecal contents (10 days after initial dose) (see Figure $3 \mathrm{E}$ ) (https://github.com/mazmanianlab/Yoo_et_al_2021/tree/main/metagenomics). In ChAT-Cre mice, Faith's phylogenetic diversity (i.e., alpha-diversity) decreased dramatically by day 9 in the feces and also in the terminal cecum sample (day 10) (Figure 5A), an outcome supported by the large number of microbial taxa that are less abundant in samples from $\mathrm{ChAT}^{+}$neuron activated mice (Figure 4 - Figure Supplements 4 and 5). In contrast, TH-Cre mice displayed similar phylogenetic diversity after activation, though there is a trend toward decreased diversity from pre-C21 to day 5 that recovers by day 9 in the feces (Figure 5A). The composition of microbial communities was determined using weighted UniFrac (i.e., beta-diversity); distances were calculated and principle coordinate analyses (PCoA) were performed. There is separation between control and activated animal groups in the feces and cecal contents of ChAT-Cre mice, a shift that was largely absent in samples from TH-Cre mice (Figure 5B-5G). Interestingly, over the experimental time course, Verrucomicrobia were significantly enriched in activated ChATCre mice (Figure $5 \mathrm{H}$ ). To uncover differentially abundant bacterial taxa, we used linear discriminant analysis effect size (LEfSe) (Segata et al., 2011) and then visualized the data into cladograms depicting the phylogenetic relationship of differentially abundant taxa (Figure $5 \mathrm{I}$ and $5 \mathrm{~K})$. Family level changes to cecal bacterial communities are displayed in Figures 5J and 5L for ChAT-Cre and TH-Cre mice, respectively. The bacterial species Akkermansia muciniphila appears to drive the increase in Verrucomicrobia in activated ChAT-Cre mice (Figure 5M) but not in TH-Cre mice (Figure $5 \mathrm{~N}$ ). A. muciniphila metabolizes host-derived mucus as a nutrient source (Derrien et al., 2004; Van Herreweghen et al., 2017), consistent with the increase in luminal mucin proteins (MUC19) and digestive enzymes in activated ChAT-Cre mice (see Figure 4A). A. muciniphila is implicated in many diseases, such as obesity (Everard et al., 2013; Plovier et al., 2017), multiple sclerosis (Cekanaviciute et al., 2017; Jangi et al., 2016), and 
seizures (Olson et al., 2018). Various additional associations can be postulated for ENS microbiome interactions from this dataset.

Beyond microbial species assignment, metagenomic analysis can reveal gene families and pathways that are differentially abundant in the microbiome. Only ChAT-Cre mice showed activation-mediated changes in beta-diversity of both gene families and pathways (Figures 50$5 R$ ), and these shifts occurred in the cecum and feces collected 9 days after activation. The most distinguishing features were highly represented in the control groups and downregulated in activated ChAT-Cre mice. The families and pathways that distinguish the groups (Figure 5 Figure Supplement 5C and 5D) were mainly associated with bacterial activity, such as nucleotide biosynthesis and metabolism, and protein translation and transport. The downregulation of these gene families and pathways in activated ChAT-Cre mice supports the corresponding decreases in bacterial alpha-diversity (see Figure 5A). We conclude that neuronal activation actively reshapes the gut microbiome at community-, species-, and geneticlevel resolutions, with considerable differences in effects between $\mathrm{ChAT}^{+}$or $\mathrm{TH}^{+}$neurons. Potential mechanisms that mediate influences by the ENS on gut bacterial composition include altered intestinal motility, regulation of immune response, control of mucus production, and shifts in metabolism / nutrient availability, among others.

\section{Metabolomic changes in the gut upon neural stimulation}

Having characterized outcomes of neuronal activation on DNA (microbial metagenome), RNA (host transcriptome), and protein (host and microbial proteome), we reasoned that a major impact of these changes may manifest in the profile of gut metabolites. Mouse and microbial cometabolism represents an intimate and intertwined relationship with implications to health. Via untargeted metabolomics using LC-MS/MS, we assayed detailed molecular changes in cecal contents and feces following neuronal activation in the gut (https://github.com/mazmanianlab/Yoo_et_al_2021/tree/main/metabolomics). In ChAT-Cre and $\mathrm{TH}$-Cre mice, we observed a strong, activation-dependent clustering of metabolome profiles in cecal samples taken one hour following the last C21 injection (Figures 6A and 6B). In longitudinal fecal samples of ChAT-Cre mice, we observed separation of metabolomes between control and activated groups by day 5 of C21 injection, a state that persisted until day 9 (Figures 6C-6F). In TH-Cre fecal samples, data were plotted on axes that minimized experimental bias from an apparent batch effect (see Methods), revealing differences between metabolomes of control and $\mathrm{TH}^{+}$neuronal activation over time (Figures 6G-6J). Furthermore, there is clear 
separation in cecal metabolites in ChAT-Cre versus TH-Cre mice, upon activation (Figure 6 Figure Supplement 6A and 6B), and such separation occurs in the feces by Day 9 of the activation time course (Figure 6 - Figure Supplement 6C-6F). Thus, targeted activation of ChAT ${ }^{+}$ and $\mathrm{TH}^{+}$neurons in the ENS strongly influences the gut metabolome.

The Global Natural Products Social Molecular Networking (GNPS) (Wang et al., 2016) tool is an open-access mass spectroscopy repository and analysis pipeline that we employed to visualize and contextualize our data. In the cecal contents of ChAT-Cre and TH-Cre mice, metabolic networks consisting of both annotated and unannotated molecules were revealed (Figures $6 \mathrm{~K}$ and 6L). Annotated, non-networked metabolites were chosen for their differential abundance in either control or activated samples. Specifically, in TH-Cre mice, there were strongly increased non-networked metabolites in activated mice whose closest spectral matches are linoelaidic acid (ID: 626), oleanolic acid methyl ester (ID: 378), and coproporphyrin I (ID: 739). Metabolites that spectrally resemble xanthine (ID: 259), genistein (ID: 846), and trans-Ferulic acid (ID: 707) were decreased upon activation (Figure 6K and Figure 6 - Table Supplement 2).

In both ChAT-Cre and TH-Cre mice, the molecular networks largely consisted of level 3 annotations (based on the Metabolomics Standards Initiative (MSI) (Sumner et al., 2007)) of compounds belonging to the bile acid molecular family and their conjugates, as well as unannotated analogs. Primary bile acids are host (mouse) derived chemicals from cholesterol biosynthesis, which are subsequently metabolized by gut bacteria into secondary bile acids (Aries et al., 1969; Sakai et al., 1980). Interestingly, metabolites with their closest spectral match to the primary bile acid cholic acid (ID: 108, 114, 215, 219, 221, 224, 259) were significantly enriched in the cecum of activated ChAT-Cre mice (Figures 6L-6N and Table s2). Additional metabolites that closely resemble the spectra of tauro-conjugated primary bile acids trended upwards, such as taurocholic acid (ID: 234, 238) and taurohyocholic acid (ID: 235). Conversely, features with spectral matches to the family of secondary bile acids and bile acid metabolites such as ursodeoxycholic acid (ID: 13), deoxycholic acid (ID: 100), beta-hyodeoxycholic acid (ID: 1, 143) and 12-ketodeoxycholic acid (ID: 19, 138) were decreased in ChAT ${ }^{+}$-activated mice (Figures $6 \mathrm{~L}-6 \mathrm{~N}$ ). These data suggest that activation of $\mathrm{ChAT}^{+}$neurons may modulate primary bile acids secretion, either directly or indirectly, and/or their metabolism to secondary bile acids.

While additional examples of host-microbial co-metabolism were detected (Table s2), the intriguing pathway alterations in bile acids following activation of $\mathrm{ChAT}^{+}$neurons may have 
critical influences on various host physiologies and health. For example, bile acids and their metabolites have historically been studied for their essential role in digesting dietary fats and regulating the absorption of lipids (de Aguiar Vallim et al., 2013) and thus have been a pharmacological target for nonalcoholic fatty liver diseases (e.g., NASH) (Gottlieb and Canbay, 2019). Bile acids regulate intestinal immune responses (Fiorucci et al., 2018; Ichikawa et al., 2012; Vavassori et al., 2009) and the production of neurotransmitters by gut enterochromaffin cells (Yano et al., 2015). Expression of bile salt hydrolases and hydratases increases the fitness of both commensal and pathogenic bacteria (Begley et al., 2005; Delpino et al., 2007; Hofmann and Eckmann, 2006; Jones et al., 2008; Sannasiddappa et al., 2017). Recently, taurocholate, a bile acid that induces expression of virulence genes in enteric pathogens, and its metabolism by the human microbiota has been linked to susceptibility and resistance to infection (Alavi et al., 2020). These extensive and important functions attributed to bile acids had not previously been known to be regulated by $\mathrm{ChAT}^{+}$neurons in the gut, a discovery that may have broad implications on nutrition, metabolism, immunity, neurotransmission, and infection.

\section{Discussion}

Brain research has outpaced exploration of other neuronal systems in the body, and led to the creation of powerful tools to study neuronal architecture and function. Curiously, many disorders of the brain are associated with gastrointestinal symptoms (Bhavsar et al., 2013; Cersosimo et al., 2013; Del Giudice et al., 1999; Pfeiffer, 2003; Valicenti-McDermott et al., 2008). While mechanisms linking the gut and the brain, and their consequences to health, are an active area of study, the impact of neuronal activation within the ENS has largely been unexplored. Knowledge of many aspects of neuronal architecture and function within the gut remain rudimentary (Carabotti et al., 2015; Furness et al., 2014). Historically, and paradoxically, early pioneers of neuroscience in the $20^{\text {th }}$ century used the ENS as a model for studying the nervous system, an approach that fell out of favor in lieu of emerging brain research.

Using a viral delivery system with enhanced infectivity of the ENS in Cre-expressing mouse models to achieve cell-specific expression of fluorescent proteins, merged with the power of tissue clearing approaches that allow 3D preservation of tissue, we report novel neuronal architectures of the myenteric plexus and submucosal plexus of mice within the same tissue preparation. Furthermore, by mapping specific neuronal sub-populations, namely $\mathrm{ChAT}^{+}$and $\mathrm{TH}^{+}$neurons, longitudinal variances along the gut and within each plexus were identified. The 
fact that these subsets do not biogeographically overlap suggests differences in functions between neurons of different classes. Further, delivery of a calcium-based activity marker allowed recording of ENS neuronal firings in live, anesthetized animals. These findings highlight the tremendous complexity of the ENS, and equip researchers with tools and approaches to further dissect structure-function relationships within the gut nervous system. Future deployment of these technologies can enable efforts to map neuronal innervations into and out of the gut, providing insights into how the ENS networks with tissues throughout the body.

Although the DREADD-based activation paradigm used in this study is inherently artificial and experimental, it provides a framework to study cellular and molecular interactions in the Gl tract that are mediated by the ENS of mice. We reveal that chronic activation of $\mathrm{ChAT}^{+}$or $\mathrm{TH}^{+}$ neurons associated with the Gl tract of mice altered the intestinal transcriptome and impacted gene expression in pathways associated with gut motility, angiogenesis, absorption, epithelial cell function, and mucosal immunity / anti-microbial responses, among others. Upon targeted activation of $\mathrm{ChAT}^{+}$or $\mathrm{TH}^{+}$neural inputs to the gut, we observed changes in genetic signatures reminiscent of numerous cell types, highlighting the integration of signals between neurons and variety of other cells in the gut. Our experimental system, and the data it engendered, do not resolve whether the transcriptomic changes are direct or indirect effects of neuronal activation. For example, activation of neuronal subset may change peristalsis, nutrient absorption, the microbiome, etc., any of which may then feedback on epithelial or immune cells to alter their gene expression profiles. Single-cell analysis may further refine connections between the ENS and the varied lineages of intestinal cells that collaborate to coordinate gut functions. Furthermore, it was previously reported that with a strong and ubiquitous promoter, PHP.S can infect other neuronal subtypes in the peripheral nervous system, such as the dorsal root ganglia. Towards this end, we utilized a weaker promoter to drive expression of DREADDs and focused the analyses on the cellular and molecular changes observed in the GI tissue and lumen. Although tissue specificity of PHP.S remains a limitation and our findings cannot be interpreted as only a direct result of ENS activation, we can associate the data as being a result of cell-type specific, neuronal activation of the Gl tract.

Ostensibly, extensive exposure to the environment requires that the intestines are charged with myriad of responsibilities including digestion and absorption of dietary nutrients, exclusion of xenobiotics, protection from enteric infection, and partnership with the gut microbiome. Indeed, we observed differences in the compositional profile of the gut microbiome between mice with 
activation of $\mathrm{ChAT}^{+}$versus $\mathrm{TH}^{+}$neurons, at the proteomic and metagenomic levels. Of note, others have shown that deletion of ChAT in enteric neurons leads to microbiome dysbiosis (Johnson et al., 2018). Our study does not shed light on how ENS activation reshapes the gut microbial community structure, which may involve altered colonic motility, changes in mucus production, and / or modulation of mucosal immune responses. One interesting host-microbial interaction appears to emerge upon activation of $\mathrm{TH}^{+}$cells, which leads to a dramatic decrease in anti-fungal chitinase (CHIA) protein expression and a concomitant bloom in fungi, suggesting that neuronal circuits regulate fungal populations in the gut. Several other hypotheses can be proposed based on our dataset for potential mechanisms by which the ENS can affect the gut microbiome.

The human gut microbiome possesses the metabolic capacity of the liver, and thus changes to both mouse gut physiology and the microbiome predictably have major influences on the gut metabolome. In a striking example of mutualism, we report widespread changes to the pool of intestinal bile acids, molecules produced via host-microbial co-metabolism. Activation of ChAT ${ }^{+}$ neurons impact expression of NPCL1 (see Figure 4A) which is involved in cholesterol transport, and cholesterol is the substrate for production of primary bile acids by mammals, which are then metabolized exclusively by the gut microbiome into secondary bile acids. Bile acids play critical roles in fat absorption (de Aguiar Vallim et al., 2013), gut motility (Kirwan et al., 1975), hormonal signaling (Watanabe et al., 2006) and immune functions (Fiorucci et al., 2018), and changes in their profiles have been associated with various disorders (McMillin and DeMorrow, 2016). While additional work is required to determine how the ENS affects levels and constitution of the bile acid pool, understanding the processes that regulate synthesis of secondary bile acids may conceivably have implications on a number of organ systems throughout the body.

The ENS adapts and responds to incredibly diverse molecular cues from the environment and must do so throughout the entire length and surface area of the intestines-the largest and most expansive internal organ with a rich network of neurons termed the "second brain" (Gershon, 2015). Exposures to molecules from the diet or the microbiome may modulate ENS function, along with extrinsic signals from outside the gut, a topic not covered here but of great significance. As a companion to ongoing brain research, the novel experimental tools, empiric datasets, and conceptual advances provided by this Resource study may stimulate research into the enteric nervous system, an endeavor with broad consequences to human health. 


\section{ACKNOWLEDGMENTS}

We thank members in the Mazmanian's laboratory for discussions throughout the research and critical reading of the manuscript. We thank Dr. Andres Collazo and Caltech's Biological Imaging Facility for training access to microscopy capabilities and the Caltech Proteome Exploration Laboratory for access to LC-MS/MS capabilities. This research was funded in whole or in part by Aligning Science Across Parkinson's ASAP-000375 through the Michael J. Fox Foundation for Parkinson's Research (MJFF). For the purpose of open access, the author has applied a CC BY 4.0 public copyright license to all Author Accepted Manuscripts arising from this submission. This work was supported by grants to S.K.M. from the Heritage Medical Research Institute, Emerald Foundation, Caltech Center for Environmental and Microbial Interactions (CEMI), the National Institutes of Health (GM007616 and DK078938), and the Department of Defense (PD160030).

\section{AUTHOR CONTRIBUTIONS}

B.B.Y. and S.K.M. designed experiments. B.B.Y. performed experiments. J.A.G., K.Y.C., C.C. (supervised by V.G.) helped in AAV-mediated ENS characterization. J.A.G. performed analysis of GCaMP6F fluorescence. P.T.B (supervised by D.W.W.), M.J.S., A.M. performed acquisition and analysis of proteomic data. V.C., G.H., G.S., Q.Z., J.S., and (supervised by R.K.) performed acquisition and analysis of metagenomic data. K.W. (supervised by P.C.D.) performed acquisition and analysis of metabolomic data. T.M.T. assisted in animal-related work. B.B.Y. and S.K.M wrote the manuscript with input from all authors. 


\section{Main Figures}

\section{Figure 1}

A.

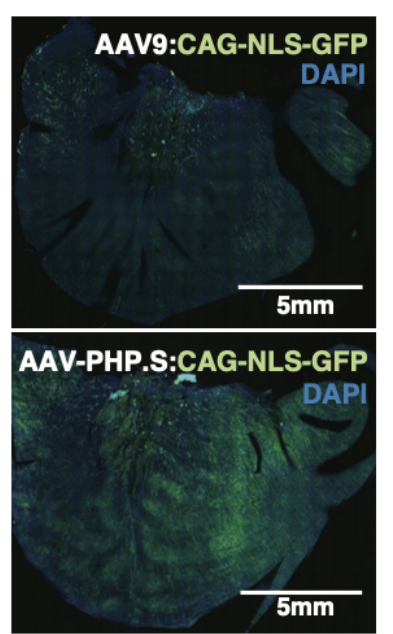

C.
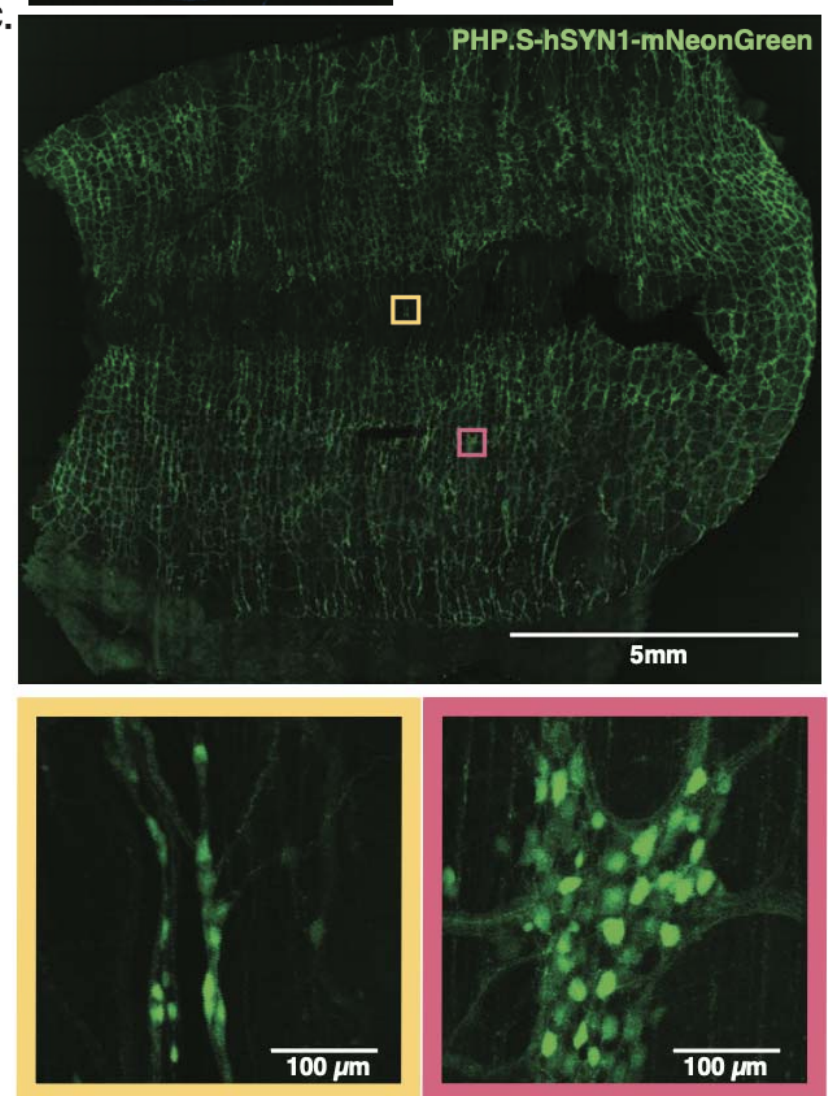

G.
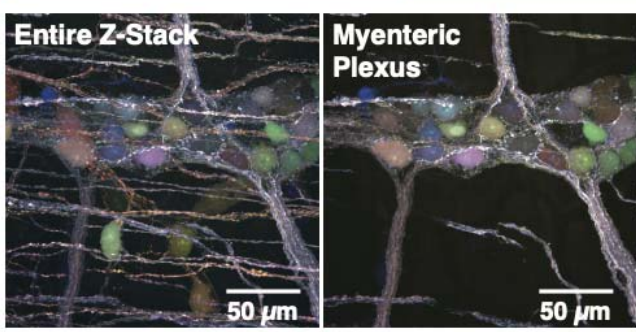

B.
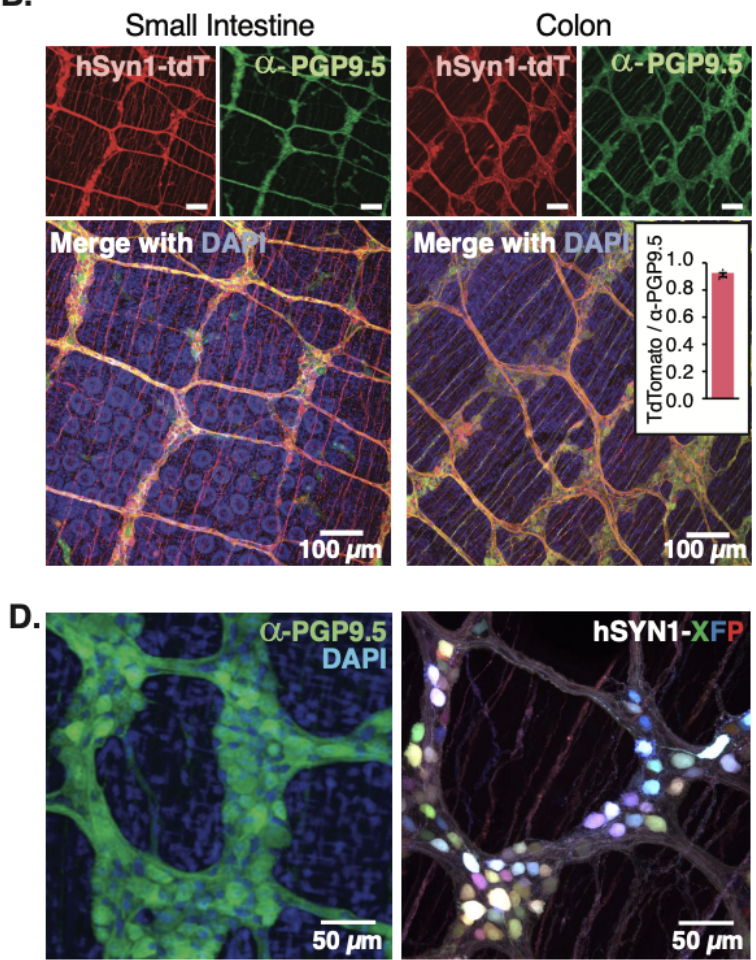

E.

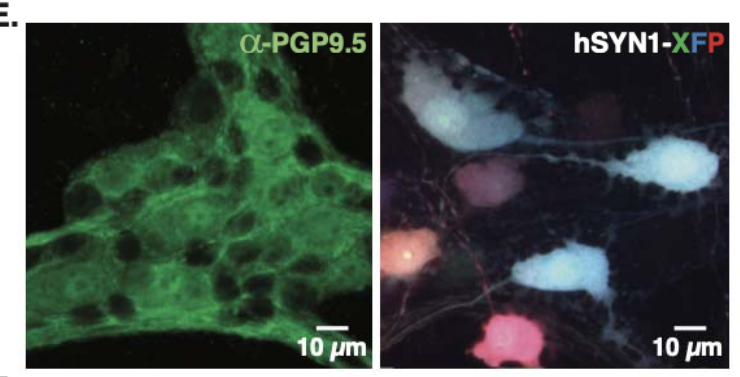

F.
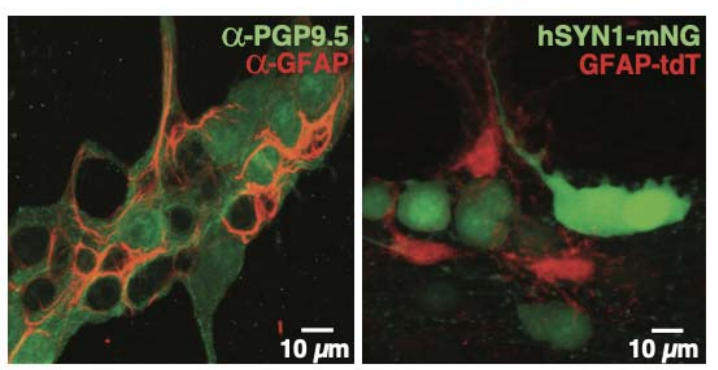

$10 \overline{\mu m}$
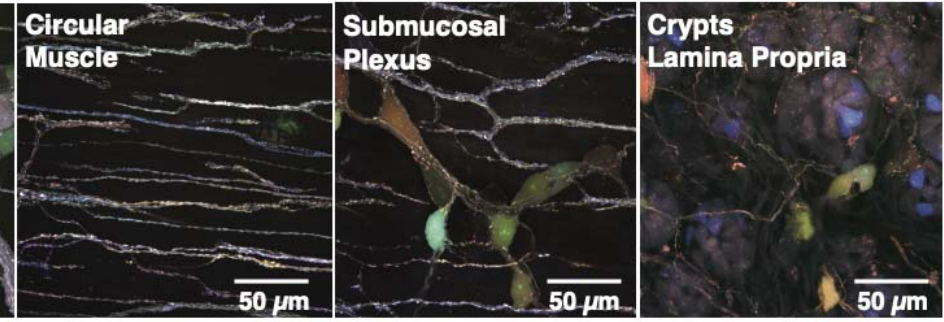
Figure 1. Advantages of Viral labeling in the ENS. (A) Viral-mediated expression of nuclear localized (NLS) GFP driven by the ubiquitous CAG promoter. Quantification of virally labelled cells in AAV9 vs. AAV-PHP.S in the longitudinal muscle, myenteric plexus, and circular muscle of the stomach. ( $\mathrm{N}=4-5$ mice, each data point represents the average from 3 representative fields). (B) Representative images of SI and colon in mice infected with AAV-PHP.S:hSYN1TdTomato and immunolabelled with the pan-neuronal antibody, PGP9.5. Inset: quantification of the ratio of $\mathrm{TdTomato}^{+}$cells / PGP9. $5^{+}$cells $(\mathrm{N}=3$ mice, each data point represents the average from 3 representative fields). (C) Tiled, whole mount microscopy showing widespread labelling in the proximal colon ( $>1 \mathrm{~cm}$ of tissue) from a single injection $\left(10^{12} \mathrm{VGs}\right.$-vector containing AAVs). Yellow inset highlights regions opposite from the mesenteric attachment; pink inset highlights a central region of the tissue. (D) Left: Antibody-labelled myenteric plexus of the proximal colon. Right: Viral-mediated multicolor labelling of a comparable region. AAV-PHP.S:hSYN1(mTurq2/mNeonGreen/mRuby2) (AAV-PHP.S:hSYN1-XFP, 3e ${ }^{11}$ VGs each). (E) High resolution confocal microscopy showing cell morphology when labelled with AAV-PHP.S:hSYN1(mTurq2/mNeonGreen/mRuby2) vs. traditional IHC with PGP9.5. (F) Left: IHC with anti-PGP9.5 and anti-GFAP Right: Viral expression of AAV-PHP.S:hSYN1-mNeonGreen and AAV-PHP.SGFAP-TdTomato. (G) PaCT tissue clearing coupled with multicolor labelling of the intestine, showing structural and anatomical resolution in $Z$.

\section{Figure Supplement 1. Viral Labeling Enables a Holistic Understanding of the ENS}

Source data 1. Source data for GFP/DAPI in Figure 1Ahttps://github.com/mazmanianlab/Yoo et al 2021/blob/main/ENS\%20quantification/aav9 vs p hps quanification.txt 


\section{Figure Supplement 1}

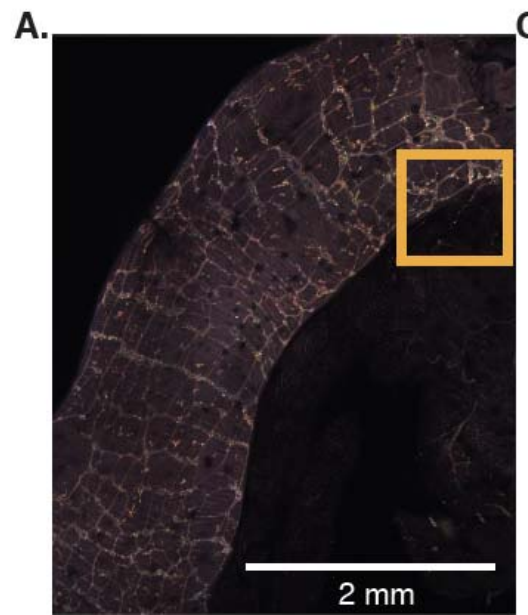

B.

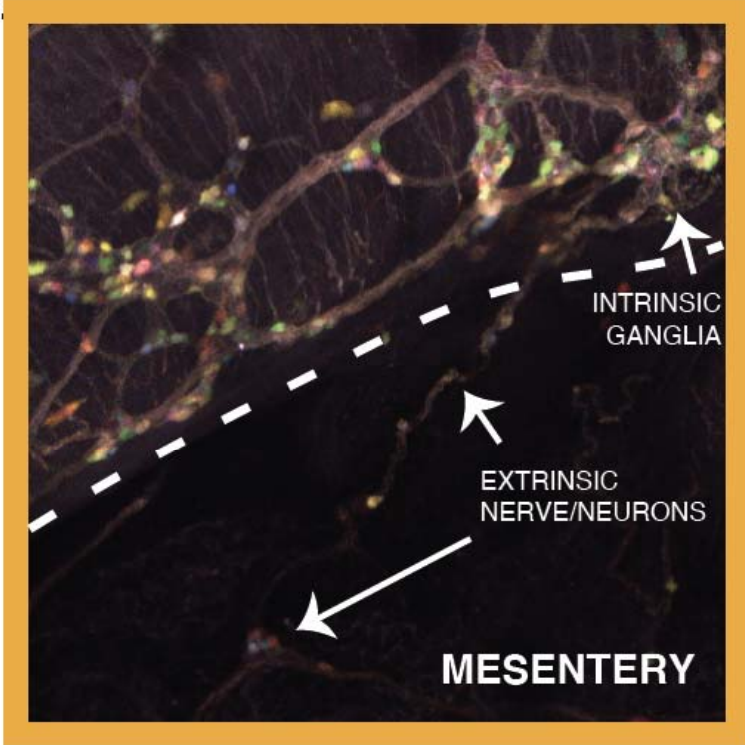

E.

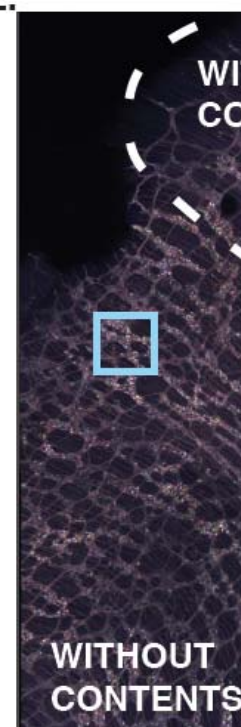

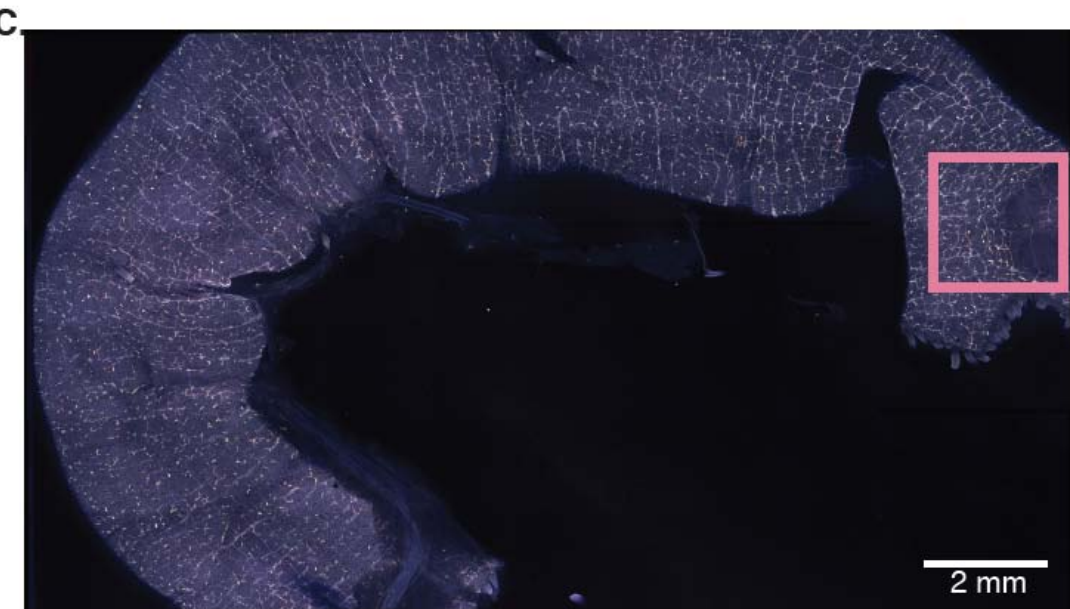

D.

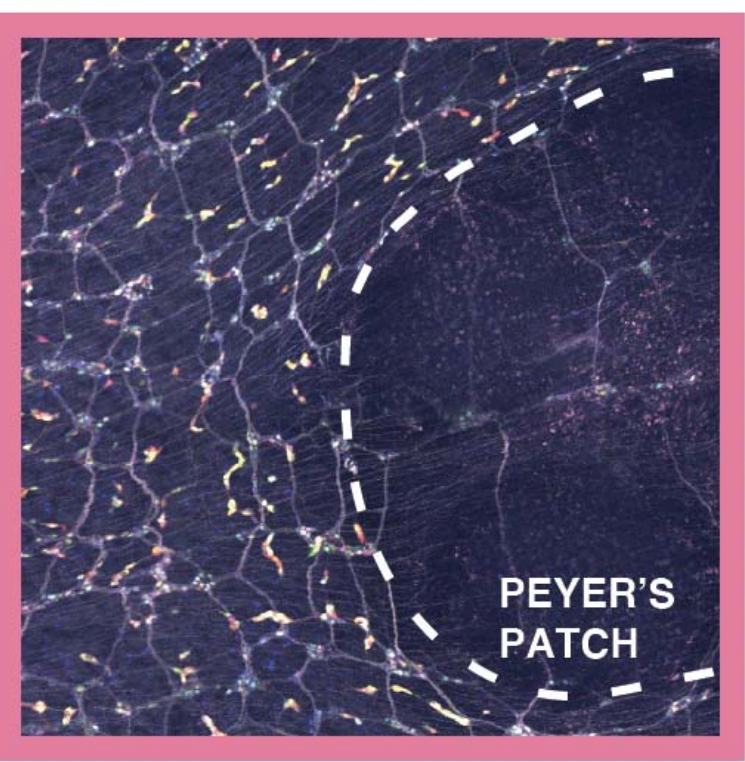

F.

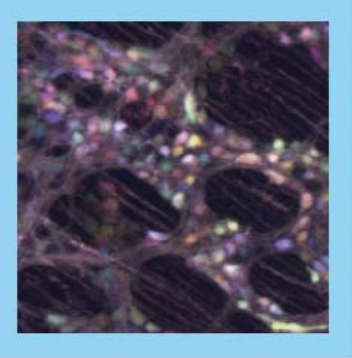

G.

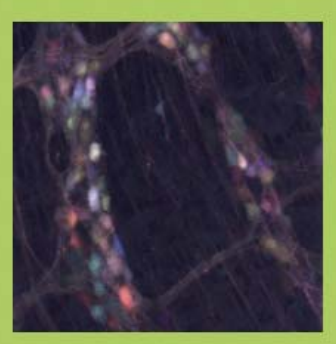


Figure Supplement 1. Viral Labeling Enables a Holistic Understanding of the ENS (Related to Figure 1). (A-D) Tiled images of the ENS in the mouse SI. Mice are infected with AAVPHP.S:hSYN1-XFP (B) Inset of panel (A) showing extrinsic nerves and neurons connecting to intrinsic ganglia of the ENS (D) Inset of panel (C) showing alterations to ENS architecture surrounding intestinal lymphoid follicles such as Peyer's patches (E-F) Tiled image of the ENS in the colon with insets showing how the $(F)$ absence or $(G)$ presence of luminal contents changes the appearance of neuronal density in the ENS. 


\section{Figure 2}

A.
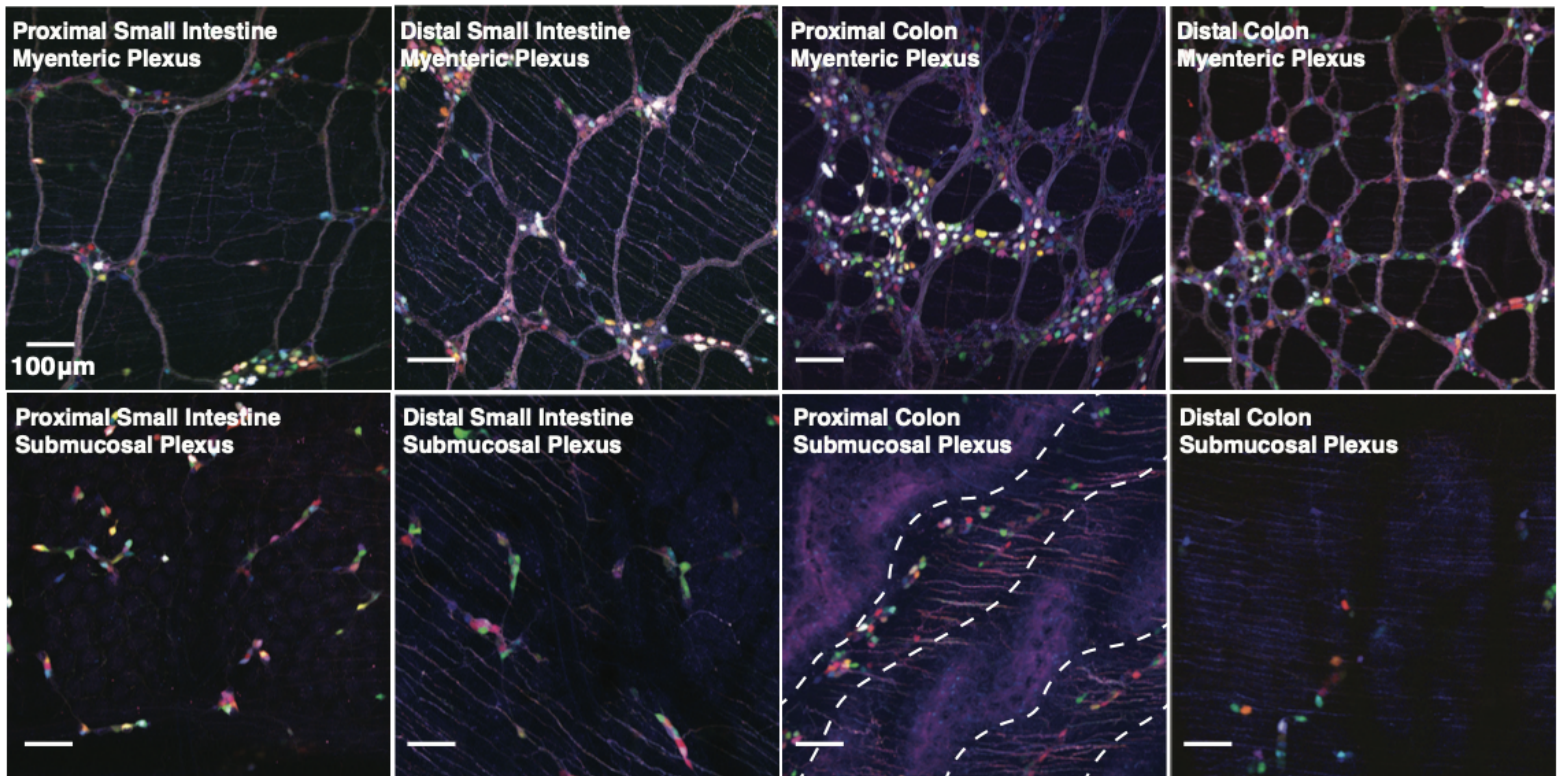

B.

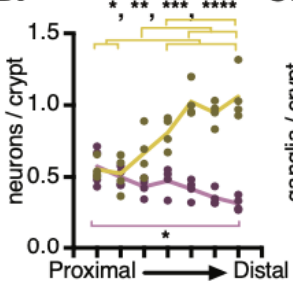

C.

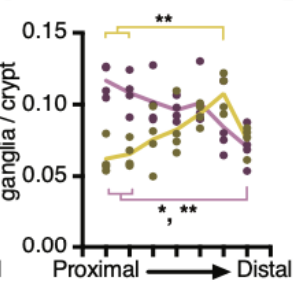

D.

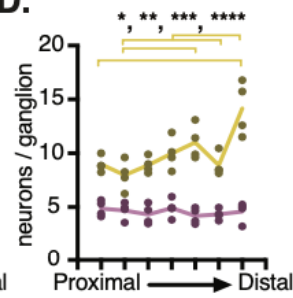

E.

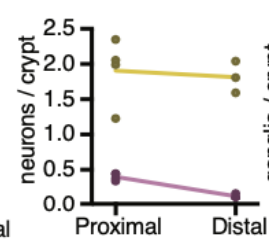

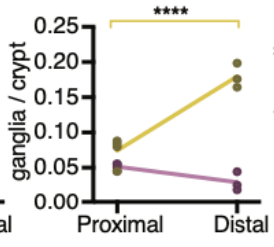

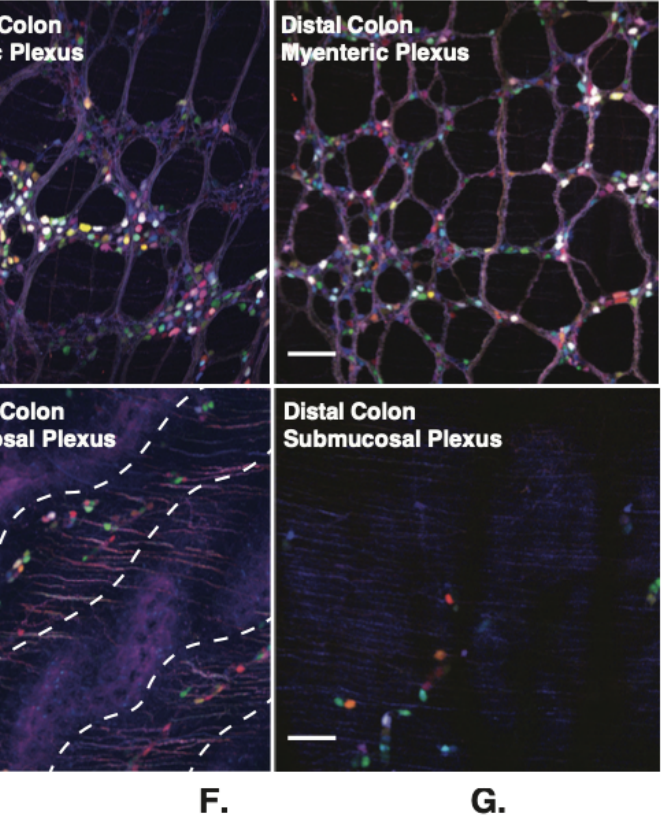

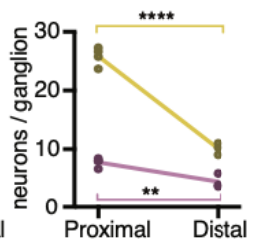

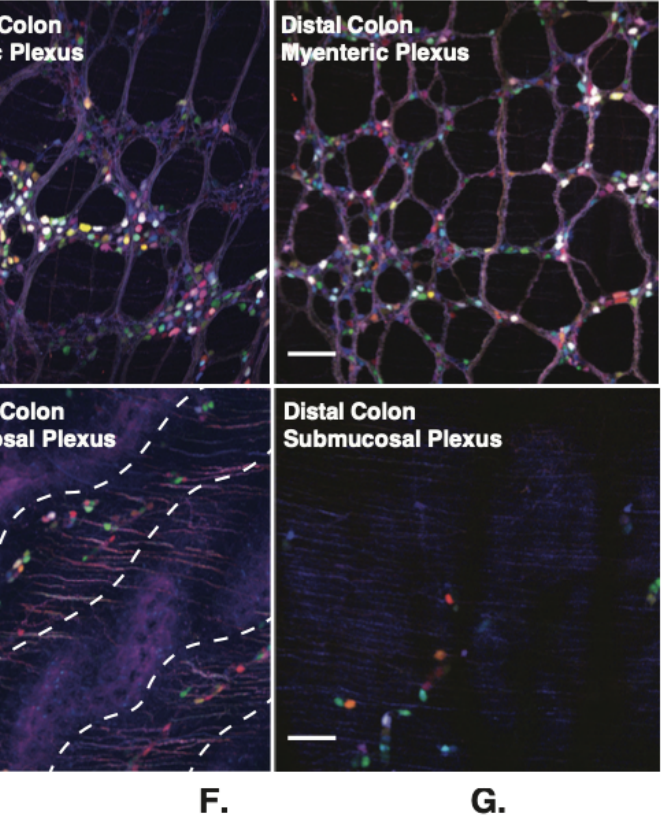

H.

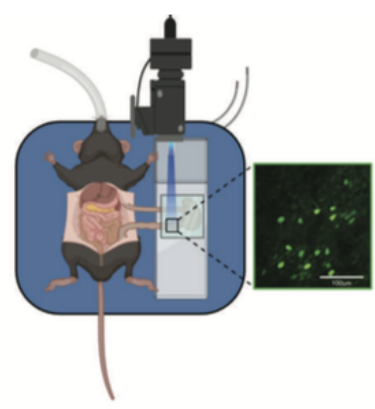

K. $10 \mathrm{sec}$

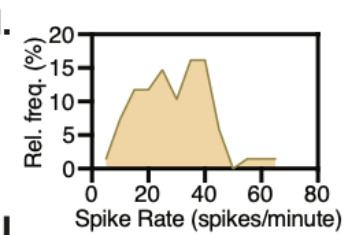

J.

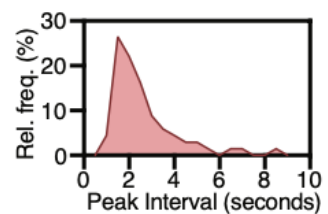

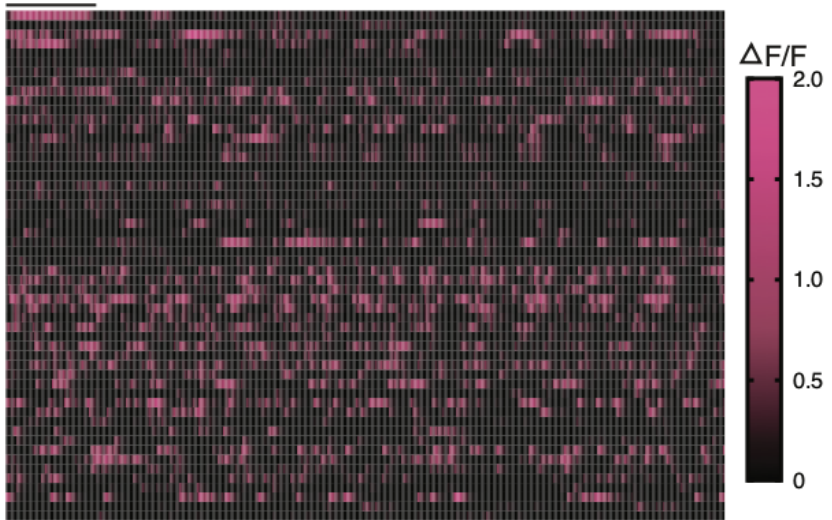

更

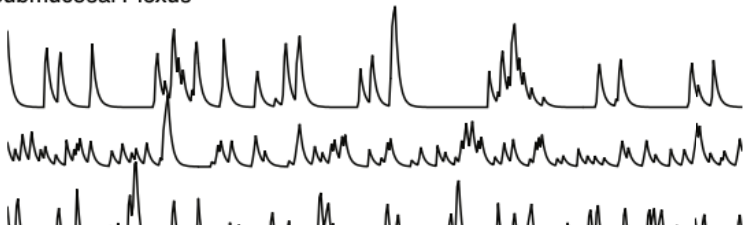

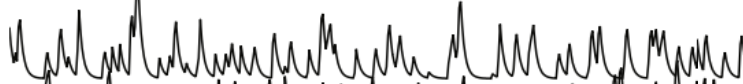

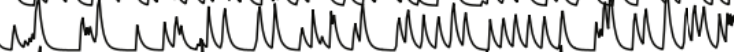

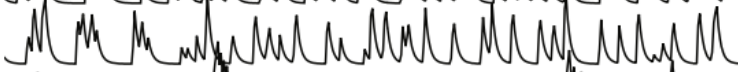

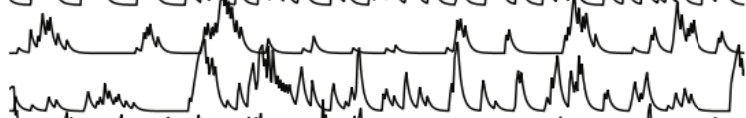

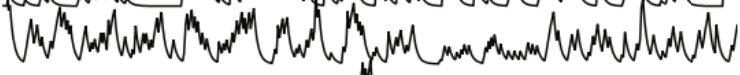

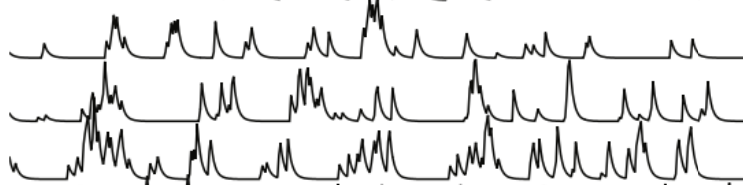

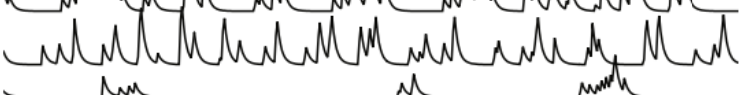

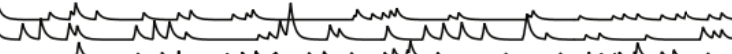
岌 S 䎡 
Figure 2. Quantifying ENS Architecture and Measuring ENS Activity with GCaMP6F. (A) Representative images from proximal and distal regions of the SI and colon from AAVPHP.S:hSYN1-XFP infected mice. Dotted lines demarcate the rugae (folds) in the proximal large intestines. (B-G) Quantification of neurons in 7 regions from the $S I$, and 2 regions from the colon, sampled proximally to distally $(\mathrm{N}=3-4$ mice per group, per intestinal region. Data points are averages from $2-3$ images per mouse, per region; ${ }^{*}: p<0.05,{ }^{* *}: p<0.01,{ }^{* * *}: p<0.001,{ }^{* * \star}$ : $p<0.001,2$-way ANOVA with Sidak's multiple comparisons test). $(H)$ Experimental setup to capture GCaMP6F fluorescence in a live, anesthetized animal. (I) Frequency distribution of calcium spike rate and $(\mathrm{J})$ peak interval (Data was collected from $\mathrm{N}=68$ cells from 3 mice). (K) Heatmap of GCaMP6F fluorescence $(\Delta F / F)$ over time. Each row represents fluorescent time course of a single cell. Data was cutoff at 83.64 seconds. (L) Representative GCaMP6F fluorescent traces.

Figure Supplement 2A-2B. Quantification of Viral DNA and Crypt Diameter in Small Intestine and Colon

Source Data 2. Source data for ENS and GCaMP6F Quantification in Figure 2B-G, 2H (Video Supplement 1), I-L, and Figure Supplement 2A-2B

Figure 2B -

https://github.com/mazmanianlab/Yoo et al 2021/blob/main/ENS\%20quantification/neurons p er crypt Sl.txt

Figure $2 \mathrm{C}$ -

https://github.com/mazmanianlab/Yoo et al 2021/blob/main/ENS\%20quantification/ganglia pe $\underline{r \text { crypt Sl.txt }}$

Figure 2D -

https://github.com/mazmanianlab/Yoo et al 2021/blob/main/ENS\%20quantification/neurons p er ganglion SI.txt

Figure 2E -

https://github.com/mazmanianlab/Yoo et al 2021/blob/main/ENS\%20quantification/neurons p er crypt colon.txt

Figure 2F -

https://github.com/mazmanianlab/Yoo et al 2021/blob/main/ENS\%20quantification/ganglia pe r crypt colon.txt

Figure 2G -

https://github.com/mazmanianlab/Yoo et al 2021/blob/main/ENS\%20quantification/neurons p er ganglion colon.txt

Figure 2H (Video Supplement 1) -

https://github.com/mazmanianlab/Yoo et al 2021/blob/main/gcamp6f/raw data/Video Supple ment\%20 1-in vivo calcium imaging.avi

Figure 2I -

https://github.com/mazmanianlab/Yoo et al 2021/blob/main/gcamp6f/peak rate gcamp.txt

Figure 2J -

https://github.com/mazmanianlab/Yoo et al 2021/blob/main/gcamp6f/peak interval gcamp.txt

Figure $2 \mathrm{~K}$ -

https://github.com/mazmanianlab/Yoo et al 2021/blob/main/gcamp6f/heatmap of gcamp6f flu orescence.txt 
bioRxiv preprint doi: https://doi.org/10.1101/2021.04.12.439539; this version posted October 15, 2021. The copyright holder for this preprint (which was not certified by peer review) is the author/funder. All rights reserved. No reuse allowed without permission.

Figure 2L (raw data for GCaMP6F fluorescence) https://github.com/mazmanianlab/Yoo et al 2021/tree/main/gcamp6f/raw data

Figure Supplement $2 \mathrm{~A}$ -

https://github.com/mazmanianlab/Yoo et al 2021/blob/main/ENS\%20quantification/relative ab undance of viral DNA gut.txt

Figure Supplement 2B -

https://github.com/mazmanianlab/Yoo et al 2021/blob/main/ENS\%20quantification/crypt diam eter gut.txt 
Figure Supplement 2

A.

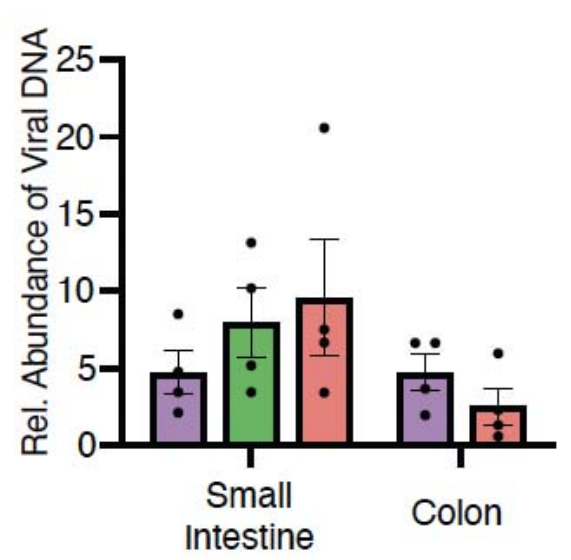

B.

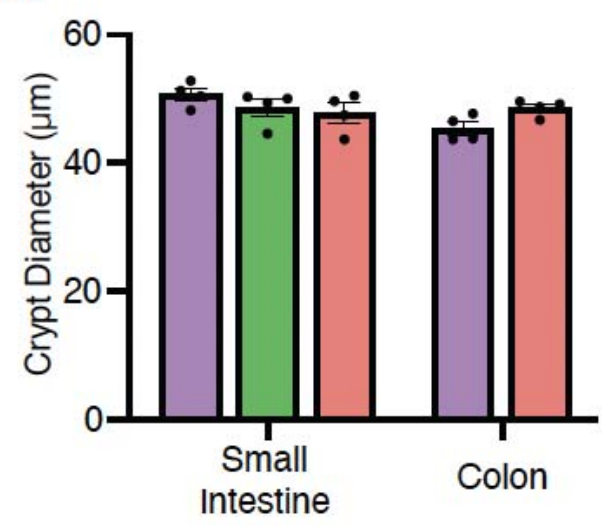

Proximal

Medial

Distal

Figure Supplement 2. Similar Viral Transduction Along the GI Tract (Related to Figure 2). (A) Quantification (qPCR) of AAV transduction in different regions of the mouse SI and colon (B) Diameter $(\mu \mathrm{m})$ of intestinal crypts in $\mathrm{SI}$ and colon ( $\mathrm{N}=3-4$ mice per group) 
Figure 3
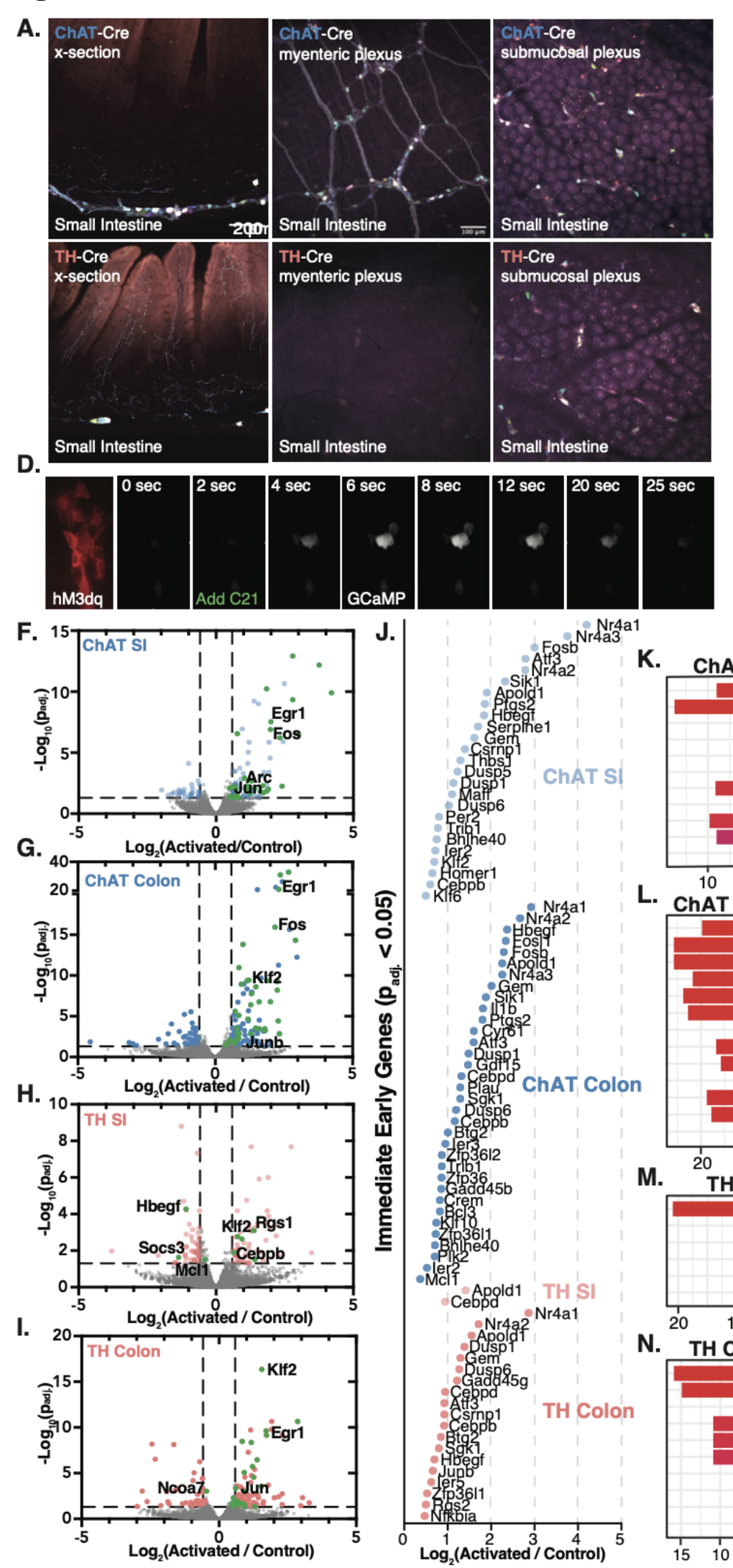

M.

N.

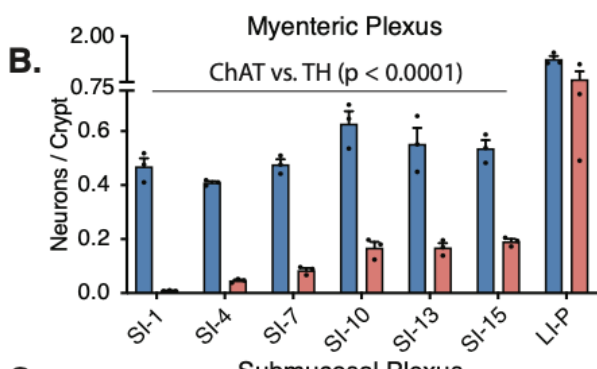

C.

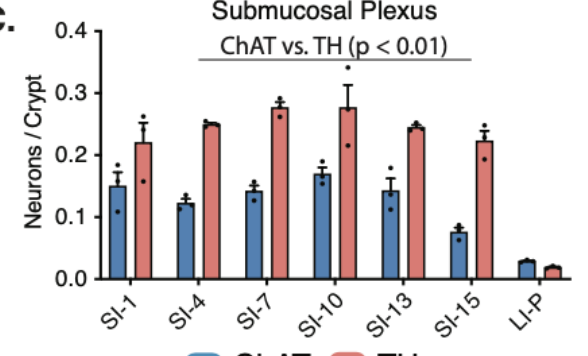

E.

Systemic C21 i.p.

Viral Delivery once per day for 10 days Endpoint
K.

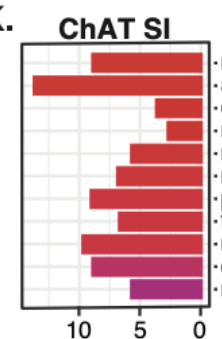

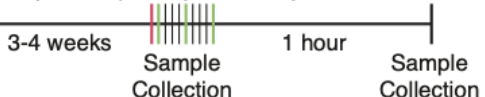

regulation of smooth muscle cell proliferation angiogenesis regulation of type B pancreatic cell proliferation regulation of epithelial cell differentiation negative regulation of protein kinase activity positive regulation of cytokine production fat cell differentiation chemotaxis response to molecule of bacterial origin

\title{
L. ChAT Colon
}

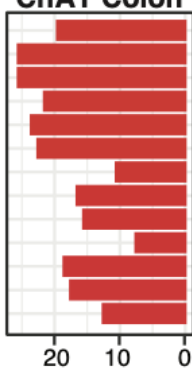

fat cell differentiation angiogenesis

response to growth factor muscle tissue development leukocyte differentiation - epithelial cell differentiation regulation of lipid biosynthetic process myeloid cell differentiation

regulation of cellular amide metabolic process cytokine metabolic process negative regulation of intracellular signal transduction lipid biosynthetic process response to peptide hormone

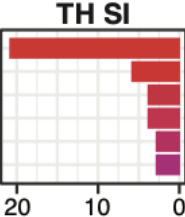

response to bacterium antimicrobial humoral response $\mathbf{p}_{\text {adj. }}$ positive regulation of sterol transport innate immune response in mucosa cellular response to copper ion positive regulation of digestive system process $\square_{0.06}$

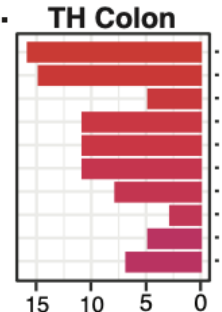

angiogenesis

-

regulation of transcription in response to stress $\mathbf{P}_{\mathrm{ad}}$ muscle tissue development

gland development

cellular response to organonitrogen compound

fat cell differentiation

branch elongation of an epithelium

cytokine metabolic process

cellular response to extracellular stimulus

$p_{\text {adj. }}<0.005$

\author{
on
}


Figure 3. Characterizing Enteric ChAT-Cre ${ }^{+}$and $\mathrm{TH}_{-}-\mathrm{Cre}^{+}$Neurons and Assaying ActivationMediated Transcriptomic Changes. (A) Representative images from cross-sections (x-section), myenteric, and submucosal plexuses in ChAT-Cre and TH-Cre mice infected with AAVPHP.S:hSYN1-DIO-XFP. (B) Density of neurons in the myenteric plexus and (C) submucosal plexus of $\mathrm{ChAT}$-Cre and $\mathrm{TH}-\mathrm{Cre}$ mice $(\mathrm{N}=3$ mice, each data point represent the average from 3 representative fields, ChAT-Cre vs. TH-Cre mice were compared using two-way ANOVAs with Sidak's correction for multiple comparisons for the SI and LI independently; Comparison of different regions in the SI of ChAT-Cre or TH-Cre mice were analyzed using one-way ANOVAs with Tukey's correction for multiple comparisons). (D) Fluorescence time course of GCaMP6F in an ex vivo preparation of ChAT-Cre SI following C21 administration. (E) Experimental paradigm10 consecutive days of C21 injections, with feces sampled in between and tissue and cecal contents collected at the end. (F-I) Volcano plots of differentially expressed genes in DREADD Activated vs Control, in (F) ChAT-Cre distal SI, (G) ChAT proximal colon, (H) TH-Cre distal SI, and (I) TH-Cre proximal colon. (Dotted vertical: Fold Change (FC)=+/- 1.5; Dotted horizontal: $\mathrm{p}_{\mathrm{adj} .<0.05}$. Annotated transcripts are IEGs and are highlighted in green. $\mathrm{N}=10$ mice per group) (J) Fold changes of upregulated IEGs $\left(p_{\text {adj. }}<0.05\right)$ as defined by Wu et al. $2017(\mathrm{~J}-\mathrm{M})$ Gene set enrichment analysis of gene ontology (GO) terms for (K) ChAT-Cre distal SI, (L) ChAT proximal colon, (M) TH-Cre distal SI, and (N) TH-Cre proximal colon.

Figure Supplement 3. Quantification of Total Neurons per Crypt and Ratios of Myenteric:Submucosal Neurons in ChAT- and TH-Cre mice.

Table Supplement 1. Expanded GO-Terms related to Figure 3K-3N https://github.com/mazmanianlab/Yoo_et_al_2021/blob/main/RNAseq/Table_Supplement_1Gene_Set_Enrichment_Analysis_of_GO_related_to_figure_3.xIsx

Source Data 3. Source Data for Figure 3B-3C (ENS Quantification for ChAT- and TH-Cre Mice), 3D (Video Supplement 2), and 3F-3J (RNA-seq fold changes)

Figure 3B -

https://github.com/mazmanianlab/Yoo et al 2021/blob/main/ENS\%20quantification/Figure 3B data\%20table myenteric per crypts.txt

Figure 3C https://github.com/mazmanianlab/Yoo et al 2021/blob/main/ENS\%20quantification/Figure 3C data table submucosal per crypts.txt

Figure 3D (Video Supplement 2) https://github.com/mazmanianlab/Yoo et al 2021/blob/main/gcamp6f/raw data/Video Supple ment 2-ex vivo calcium imaging plus C21.avi

Figure 3F https://github.com/mazmanianlab/Yoo et al 2021/blob/main/RNAseg/CHAT SI differentially e xpressed genes volcano.txt

Figure 3G https://github.com/mazmanianlab/Yoo et al 2021/blob/main/RNAseq/CHAT colon differentiall y expressed genes volcano.txt

Figure $3 \mathrm{H}$ https://github.com/mazmanianlab/Yoo et al 2021/blob/main/RNAseq/TH SI differentially expr essed genes volcano.txt

Figure 3I https://github.com/mazmanianlab/Yoo et al 2021/blob/main/RNAseq/TH colon differentially e xpressed genes volcano.txt 
Figure 3J -

https://github.com/mazmanianlab/Yoo et al 2021/blob/main/RNAseq/fold changes of upregul ated IEGs\%20.txt

Figure Supplement $3 A$ -

https://github.com/mazmanianlab/Yoo et al 2021/blob/main/ENS\%20quantification/Supplemen tal Figure 3A total\%20neurons per crypt.txt

Figure Supplement 3B -

https://github.com/mazmanianlab/Yoo et al 2021/blob/main/ENS\%20quantification/Supplemen tal Figure 3B myenteric submucosal ratio.txt 
Figure Supplement 3

A.

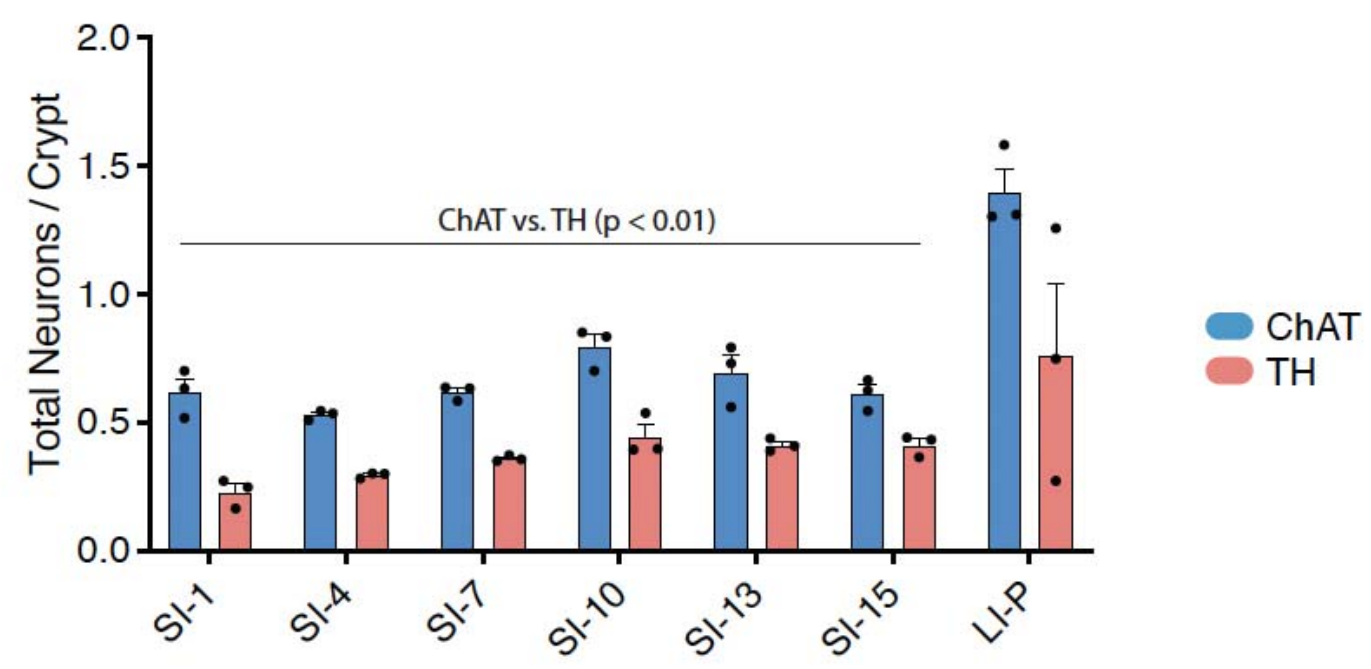

B.

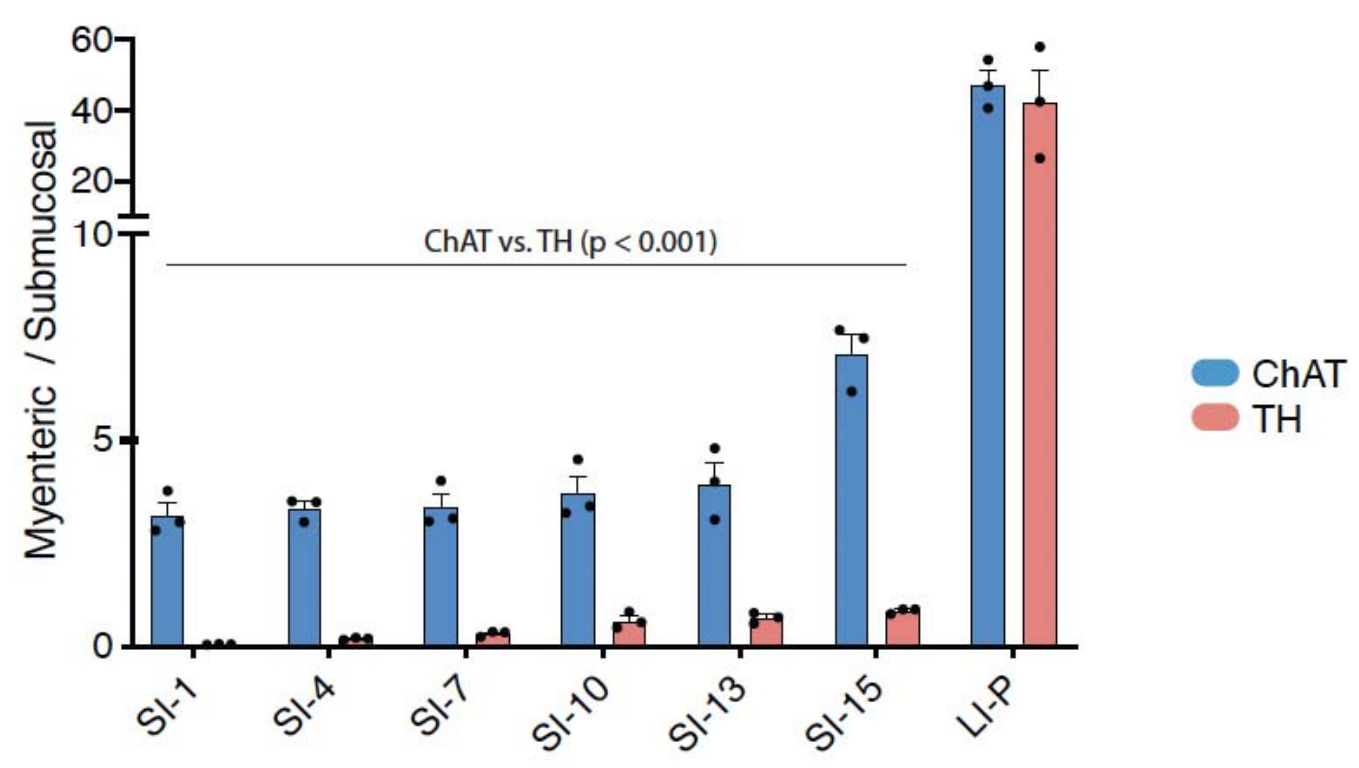

Figure Supplement 3. Quantification of Total Neurons per Crypt and Ratios of Myenteric:Submucosal Neurons in ChAT- and TH-Cre mice (Related to Figure 3). (A) Quantification of total neurons normalized by the number of crypts (B) Quantification of myenteric : submucosal cell ratio in ChAT-Cre and $\mathrm{TH}$-Cre mice. This data is an alternate representation of what is seen in Figure $3 \mathrm{~B}$ and $3 \mathrm{C}(\mathrm{N}=3$ mice, each data point represents the average from 3 representative fields; ChAT-Cre vs. TH-Cre mice were compared using two-way ANOVAs with Sidak's correction for multiple comparisons for the SI and LI independently). 


\section{Figure 4}

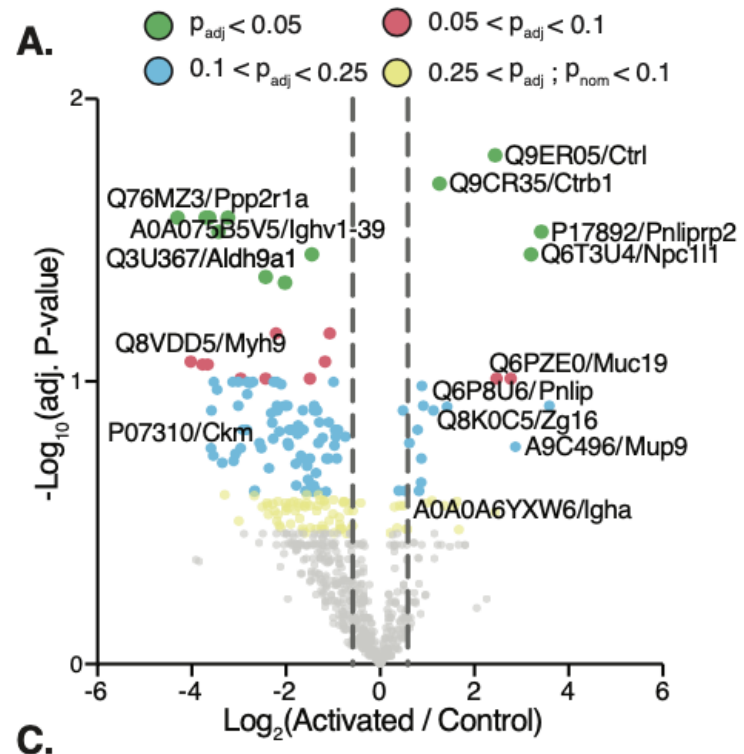

c.
B.
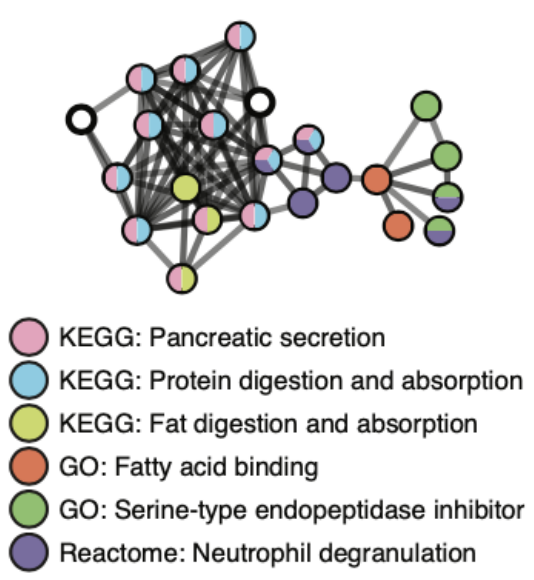

D.
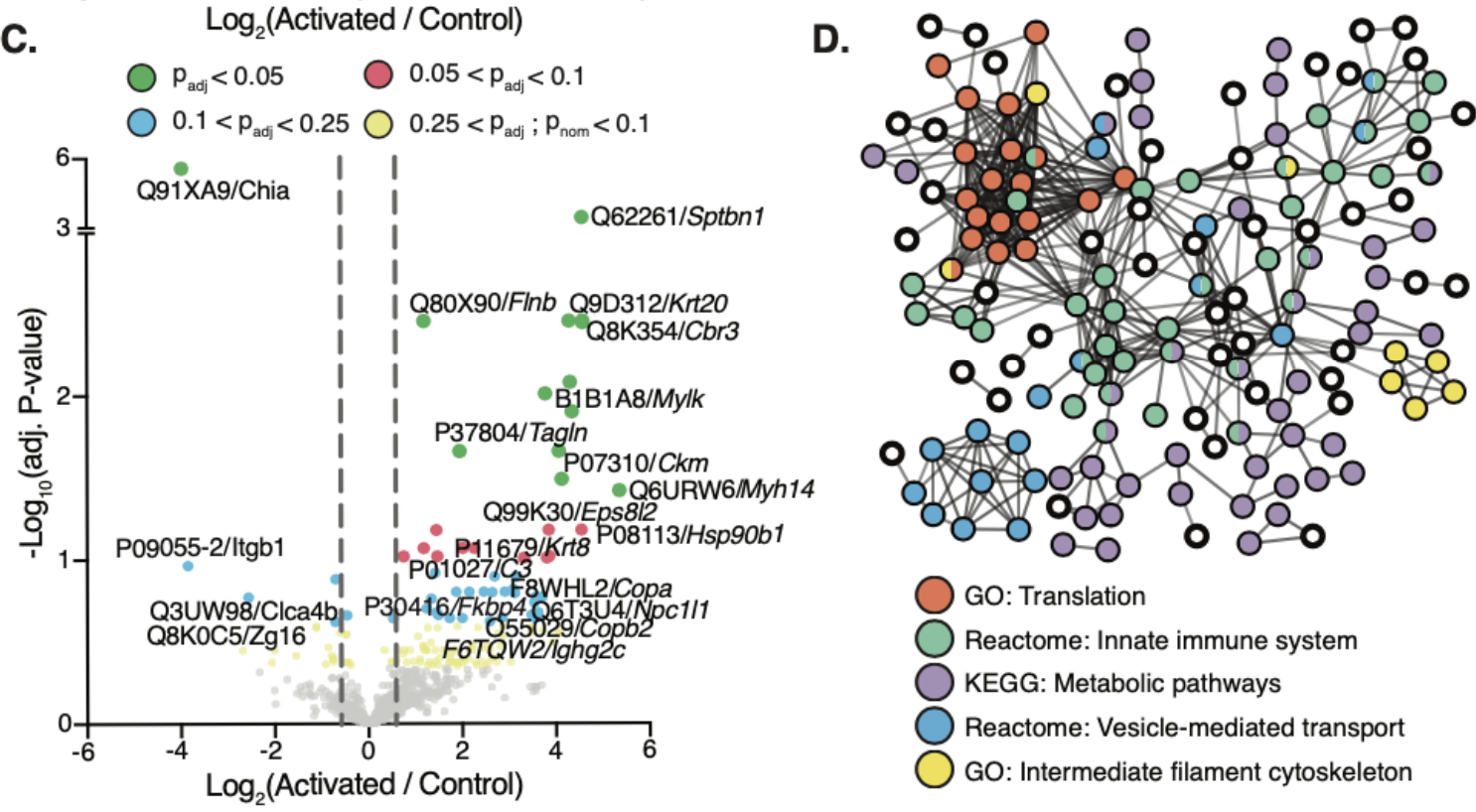

E.

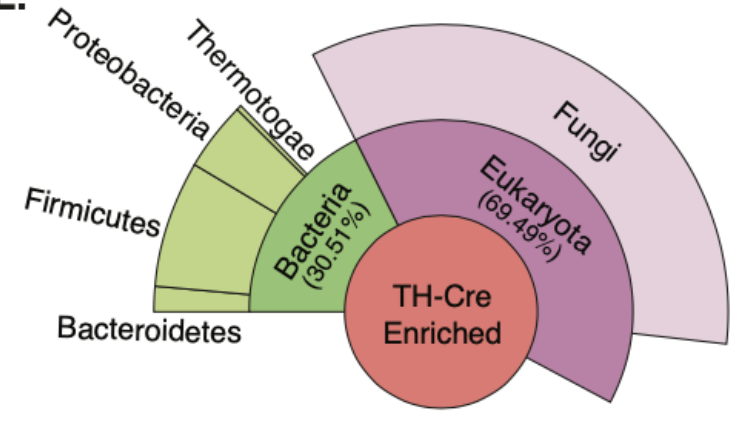

F.

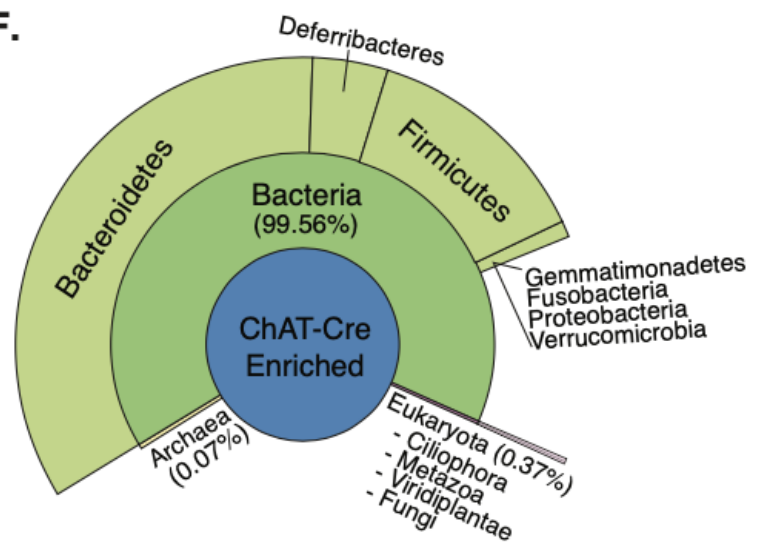


Figure 4. ENS Activation Alters Host and Microbe-Derived Luminal Proteins. (A) Volcano plot of differentially expressed host proteins identified in the cecal contents of Activated ChAT $(\mathrm{N}=8)$ vs. Control ChAT mice ( $\mathrm{N}=9$ mice), 1 hour after $\mathrm{C} 21$ administration. (B) STRING network

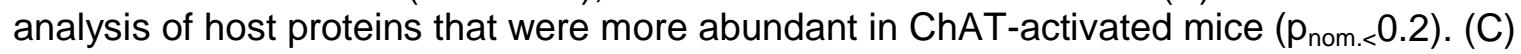
Proteomic volcano plots of $\mathrm{TH}$-activated vs. TH-control mice ( $\mathrm{N}=7$ mice per group) after $\mathrm{C} 21$ administration (D) STRING network analysis of upregulated host proteins in TH-activated mice $\left(p_{\text {nom.< }} 0.2\right)$. (E-F) Unipept metaproteomic analysis of upregulated microbial proteins (Fold Change $\left.>2, p_{\text {nom. }}<0.2\right)$ in activated $(E)$ TH and (F) ChAT-Cre mice (N=7-9 mice per group).

Source Data 4. Source data for Figure 4A-4F

Figure 4A -

https://github.com/mazmanianlab/Yoo et al 2021/blob/main/proteomics/CHAT proteomics vol $\underline{\text { cano.txt }}$

Figure 4C -

https://github.com/mazmanianlab/Yoo et al 2021/blob/main/proteomics/TH proteomics volcan $\underline{\text { o.txt }}$

Figure 4E and 4F -

https://github.com/mazmanianlab/Yoo et al 2021/blob/main/proteomics/metaproteomics/Micro biome\%20associated\%20proteins.xlsx 
bioRxiv preprint doi: https://doi.org/10.1101/2021.04.12.439539; this version posted October 15, 2021. The copyright holder for this preprint (which was not certified by peer review) is the author/funder. All rights reserved. No reuse allowed without permission.

Figure 5

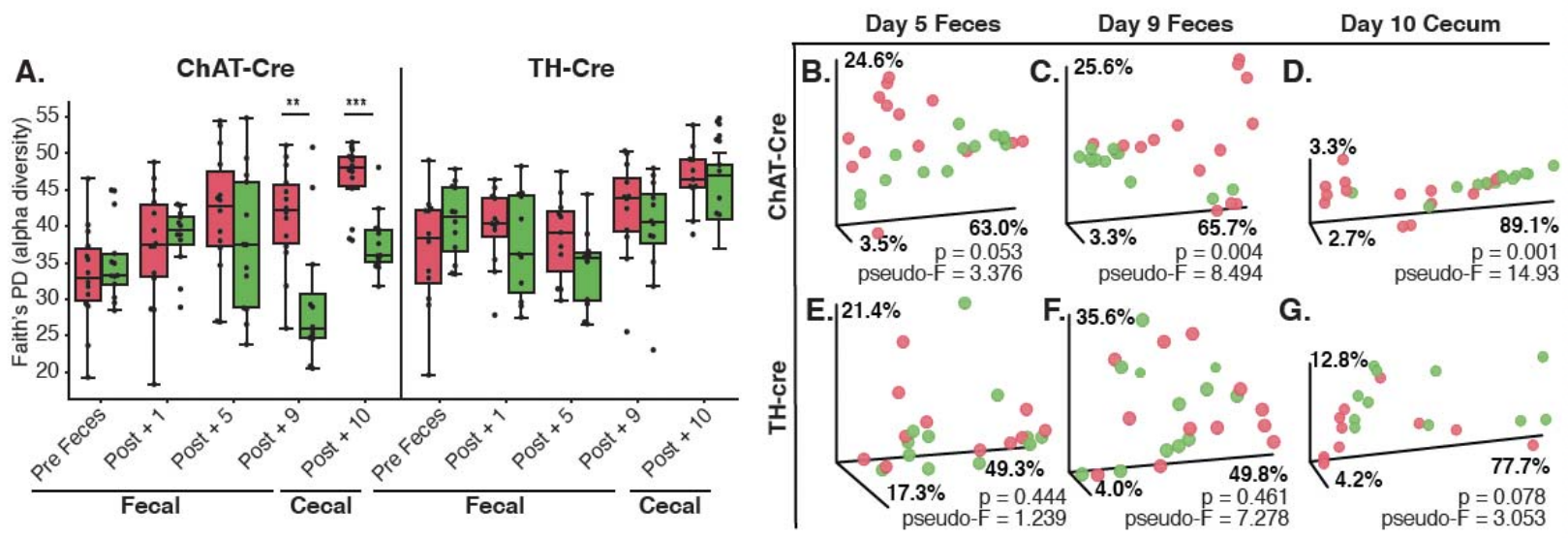

H.

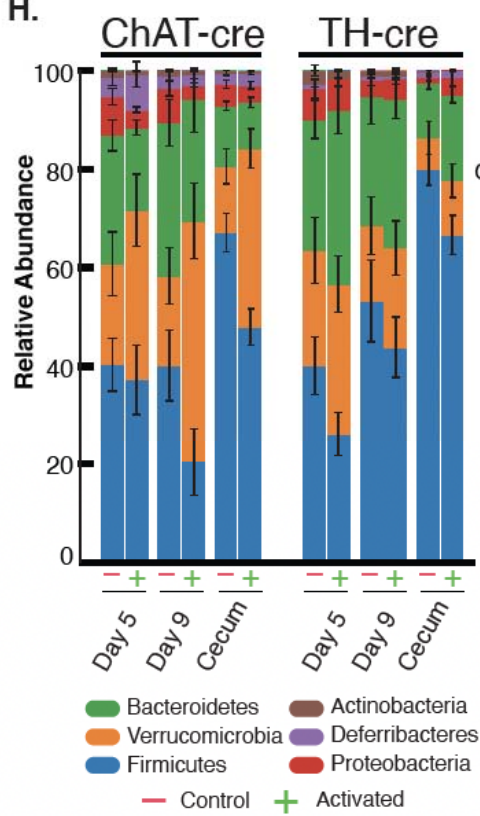

I. $\hat{3}_{\hat{i},}$

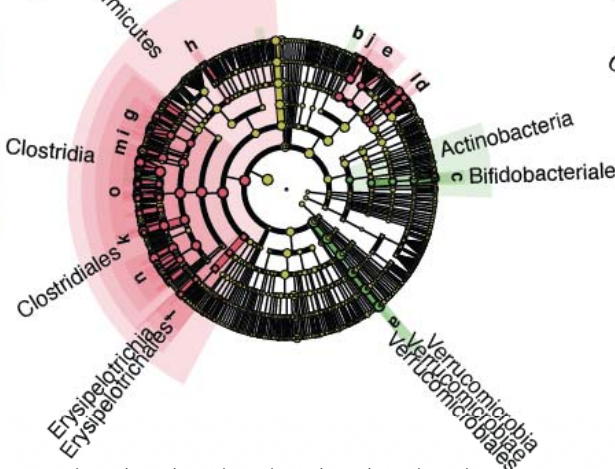

J.

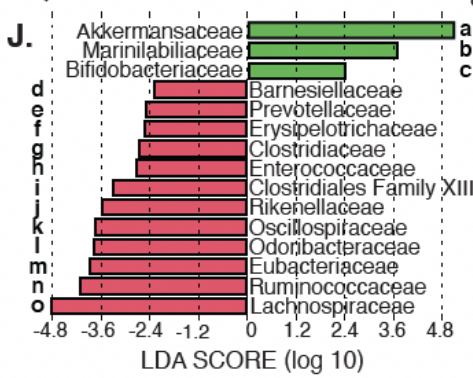

k.

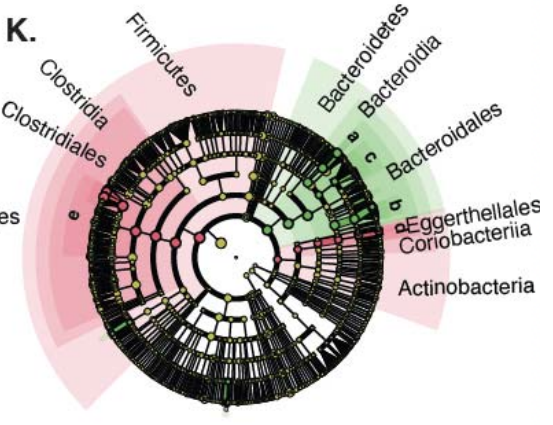

M.

M. ChAT-Cre

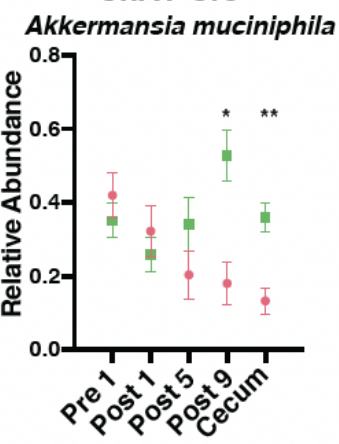

N.

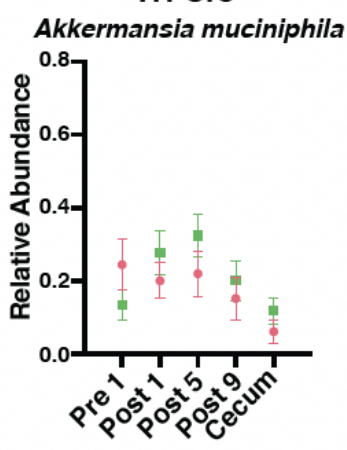

L.

a

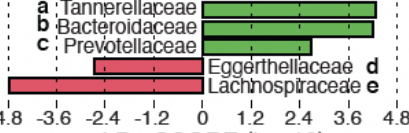

$\begin{array}{lllllllll}-4.8 & -3.6 & -2.4 & -1.2 & 0 & 1.2 & 2.4 & 3.6 & 4.8\end{array}$ LDA SCORE (log 10)

Enriched in:

Control $\bigcirc$ Activated Gene Famlly

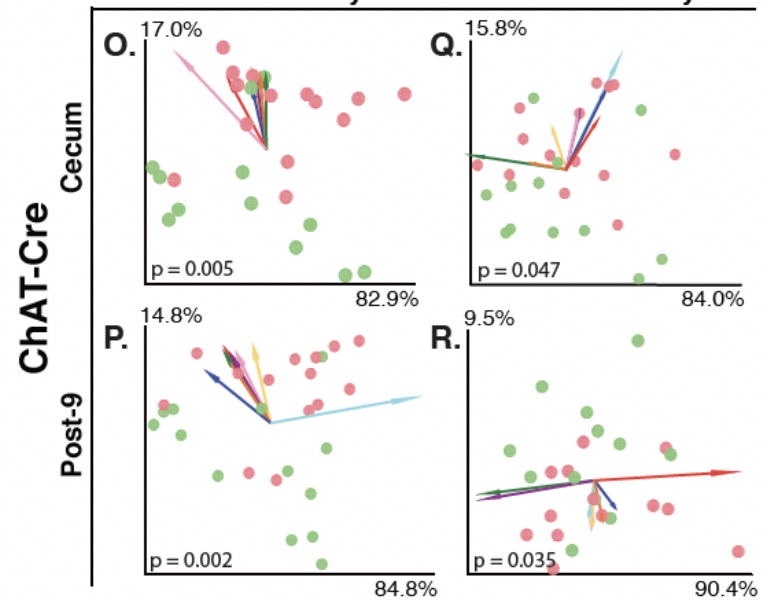


Figure 5. ENS Activation Alters the Gut Microbiome. (A) Faith's phylogenetic diversity of feces and cecal contents over 10 days of neural activation in ChAT- and TH-Cre mice. Pre (1 day before first injection), Post $+1 / 5 / 9$ (1, 5, 9 days after first injection), Post +10 (Cecal contents collected at experimental endpoint) $\left({ }^{* *} p<0.01,{ }^{* *} p<0.001\right.$ determined by Kruskal-Wallis oneway ANOVA). (B-G) Weighted UniFrac principle coordinate analysis (PCoA) of Activated vs. Control in ChAT- and TH-Cre mice with PERMANOVA p-values and pseudo-F e analyses were performed in QIIME2 as done previously (Bolyen et al., 2019). (H) Stacked bar graph showing phylum-level changes in relative abundance, 5 and 9 days post-injection in feces and 10 days post-injection in cecal contents. (I-L) Cecal Microbiome LDA Effect Size (LEfSe) Analysis: (I,K) Cladograms showing differential phylogenetic clusters and $(\mathrm{J}, \mathrm{L})$ LDA plots of family-level differences between Activated and Control mice (Cutoff: $\log _{10}($ LDA Score) $>2$ or $<-2$ ) in $(I, J)$ ChAT-Cre and (K,L) TH-Cre mice. (M-N) Changes in relative abundance of Akkermansia muciniphila in feces and cecal contents of $(\mathrm{M})$ ChAT-Cre and $(\mathrm{N}) \mathrm{TH}$-Cre mice. $(\mathrm{n}=11-14$ mice per group, per time point; red=Control, green=hM3Dq-Activated, ${ }^{*} p<0.05$, ${ }^{* *} p<0.01$, significance determined by multiple t-tests with Holm-Sidak correction for multiple comparisons). (O-R) Betadiversity of bacterial gene families and pathways in the (O-Q) cecum and (P-R) post- 9 feces of control and activated mice. Beta-diversity was determined with Robust Aitchison Principle Component Anaylsis (PCA) and analyzed using the DEICODE toolbox. The direction of defining features is indicated with colored arrows. The colors of the arrows represent different gene families and pathways and their length and direction is indicative of the influence that each feature has on the separation of control and activated groups. These gene families and pathways are annotated by the color of the feature arrow (Figure Supplement 5C and 5D).

Figure Supplement 4 - Species level taxonomic changes by LEfSe related to Figure 3I and 3K Figure Supplement 5 - LEfSe cladograms on feces after neuronal activation and microbial gene family annotations relating to Figure 30-3R

Source Data 5. Source Data for Figure 5

Figure $5 \mathrm{H}$ -

https://github.com/mazmanianlab/Yoo et al 2021/blob/main/metagenomics/LEVEL 2 META YOO2.csv

Figure $5 \mathrm{I}-5 \mathrm{~L}$ -

https://github.com/mazmanianlab/Yoo et al 2021/tree/main/metagenomics/LEfSe

Figure $5 \mathrm{M}$ -

https://github.com/mazmanianlab/Yoo et al 2021/blob/main/metagenomics/CHAT a muciniphi la timecourse.txt

Figure $5 \mathrm{~N}$ -

https://github.com/mazmanianlab/Yoo et al 2021/blob/main/metagenomics/TH a muciniphila timecourse.txt 
bioRxiv preprint doi: https://doi.org/10.1101/2021.04.12.439539; this version posted October 15, 2021. The copyright holder for this preprint (which was not certified by peer review) is the author/funder. All rights reserved. No reuse allowed without permission.

Figure Supplement 4

A.

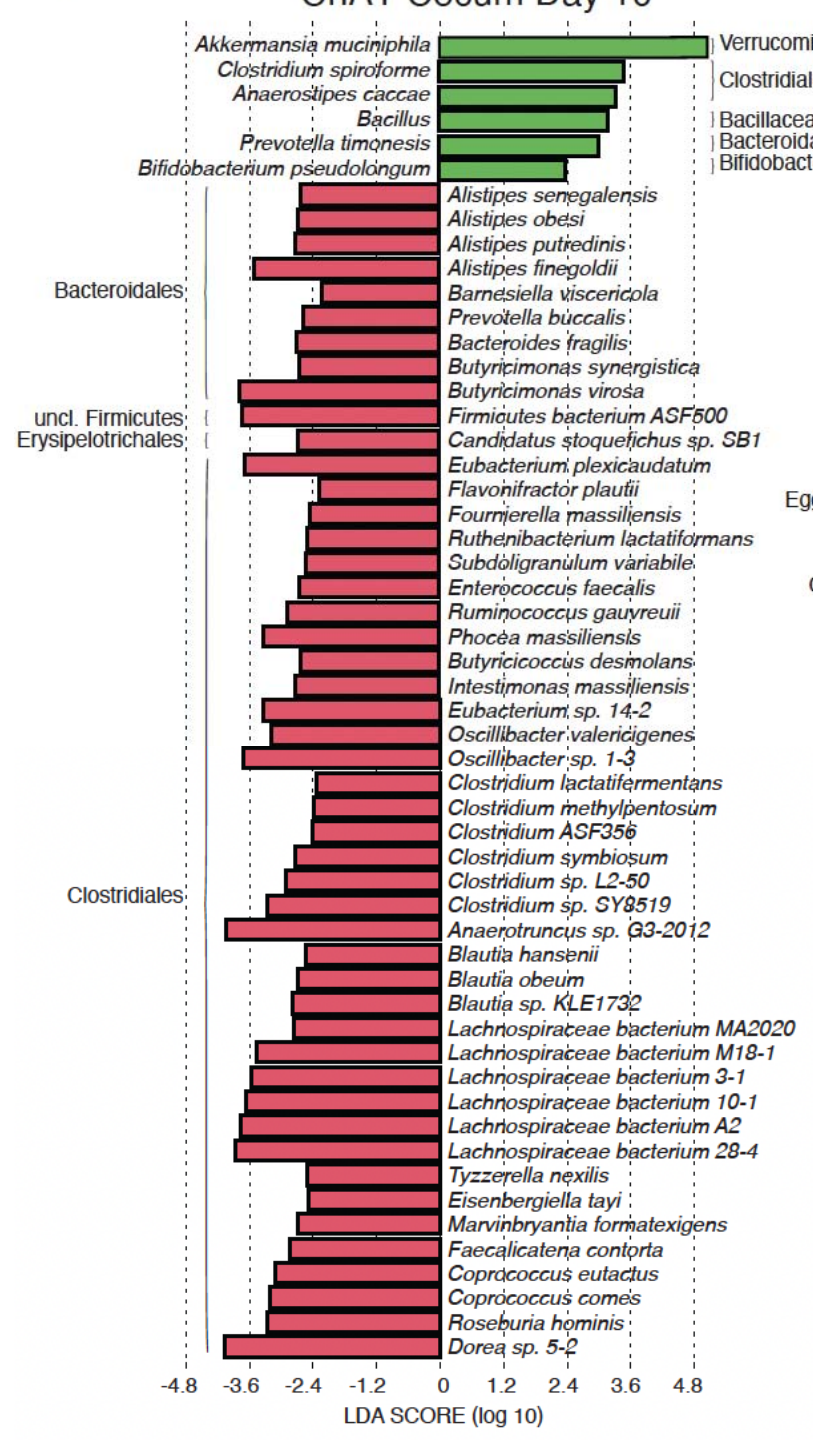

B. TH Cecum Day 10

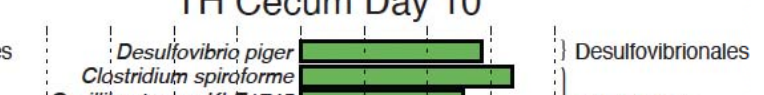
Oscillibacter sp. KLE1745 Ruminocóccus ginavus Intestimona's butyriciproducens Parabacteroides goldsteinii Odoribacter laneus Alistipes times, obesi Alistipés timonensis Alistipes śenegálensis Bacteroides uniformis
Bacteroldes vullgatus Bacteroldes vullgatus
Biacteroides stercoris Baçteroides salyersiae Bacteroides pyógenes Bacteroides thetaiotamicron

Prevotella dentalis

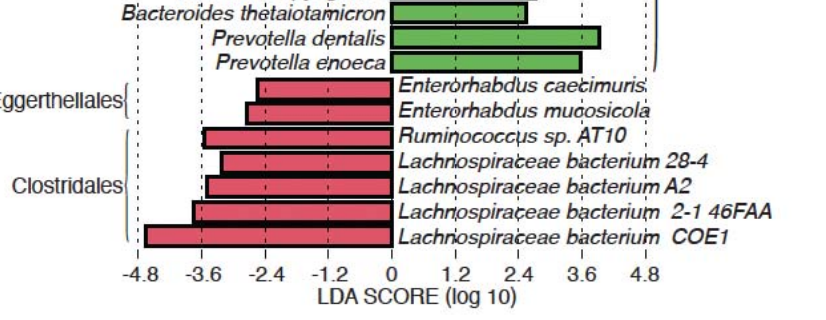

Enriched in:

Control Activated

Figure Supplement 4. Annotated changes to gut bacteria (Related to Figure 5). (A,B) Specieslevels differences in cecal bacteria corresponding to main text Figure 5I-5L. (A) ChAT-Cre and (B) TH-Cre ceca, day 10 after initial C21 injection. 
Figure Supplement 5

A.

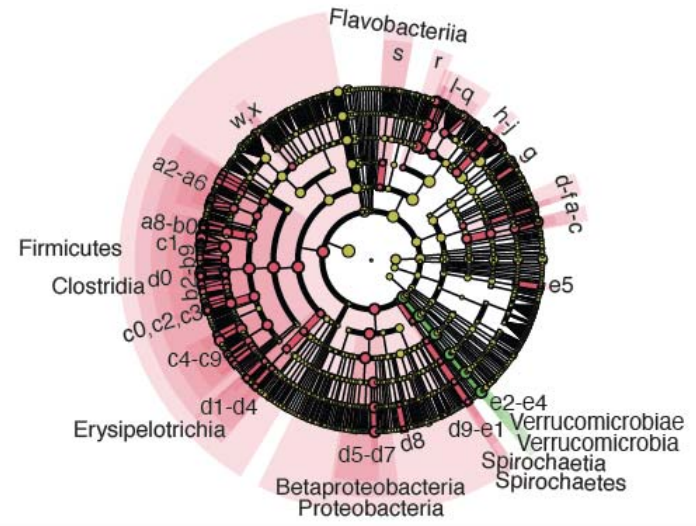

\section{ChAT-Cre Post 9}

\begin{tabular}{|c|c|c|c|c|c|c|c|}
\hline \multicolumn{8}{|c|}{ ChAT-Cre Post 9} \\
\hline ID & Microbial Taxon & ID & Microbial Taxon & ID & Microbial Taxon & ID & Microbial Taxon \\
\hline a & Bifidobacterium & $\mathrm{p}$ & Parabacteroides & b4 & Dorea & d0 & Clostridiales \\
\hline b & Bifidobacteriaceae & $q$ & Tannerellaceae & b5 & Eisenbergiella & d1 & Candidatus Stoquefichus \\
\hline c & Bifidobacteriales & $r$ & Marinilbiliales & b6 & Faecalicatena & d2 & Faecalitalea \\
\hline d & Cellulomonas & $\mathrm{s}$ & Flavobacteriales & b7 & Lachnoclostridium & d3 & Erysipelotrichaeceae \\
\hline e & Cellulomondaceae & w & Enterococcus & b8 & Marvinbryantia & $\mathrm{d} 4$ & Erysipelotrichales \\
\hline$f$ & Micrococcales & $\mathrm{x}$ & Enterococcaceae & b9 & Sellimonas & d5 & Parasutterella \\
\hline $\mathrm{g}$ & Adlercreutzia & a2 & Intestinimonas & c0 0 & Tyzzerella & d6 & Sutterellaceae \\
\hline $\mathrm{h}$ & Barnsiella & a3 & Pseudoflavonifractor & c1 & Lachnospiraceae & d7 & Burkholderiales \\
\hline $\mathrm{i}$ & Coprobacter & a5 & Clostridium & c2 & Oscillibacter & d8 & Lawsonia \\
\hline j & Barnesiellaceae & a6 & Clostridiaceae & c3 & Oscillospiraceae & d9 & Treponema \\
\hline $\mathrm{k}$ & Prevotella & a8 & Anaerofustis & c5 & Anaerotruncus & $\mathrm{e} 0$ & Spirochaetaceae \\
\hline 1 & Prevotellaceae & a9 & Eubacterium & c6 & Ruminiclostridium & e1 & Spirochaetales \\
\hline $\mathrm{m}$ & Alistipes & bo & Eubacteriaceae & c7 & Ruminococcus & e2 & Akkermansia \\
\hline $\mathrm{n}$ & Rikenella & b2 & Blautia & c8 & Ruthenibacterium & e3 & Akkermansiaceae \\
\hline o & Rikenellaceae & b3 & Coprococcus & c9 & Ruminococaceae & e4 & Verrucomicrobiales \\
\hline & & & & & & e5 & Varicellovirus \\
\hline
\end{tabular}

B.

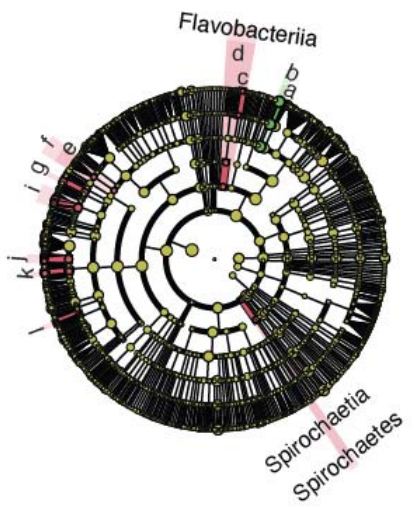

\begin{tabular}{|l|l|}
\hline \multicolumn{2}{|c|}{ TH-Cre Post 5 } \\
\hline ID & Microbial Taxon \\
\hline a & Parabacteroides \\
\hline b & Tannerellaceae \\
\hline c & Fluviicola \\
\hline d & Flavobacteriales \\
\hline e & Streptococcus \\
\hline f & Streptococcaceae \\
\hline g & Pseudoflavonifractor \\
\hline i & Clostridium \\
\hline j & Blautia \\
\hline k & Eisenbergiella \\
\hline I & Clostridiodes \\
\hline
\end{tabular}

\section{Enriched in:}

Control

Activated c.

\begin{tabular}{|c|c|l||}
\hline \multicolumn{3}{|c|}{ ChAT-Cre Cecum } \\
\hline ID & Gene Family & \multicolumn{1}{|c|}{ Annotation } \\
\hline 1 & K02003 & putative ABC transport system ATP-binding protein \\
\hline 2 & K02518 & translation initiation factor IF-1 \\
\hline 3 & K02948 & small subunit ribosomal protein S11 \\
\hline 4 & K02950 & small subunit ribosomal protein S12 \\
\hline 5 & K02965 & small subunit ribosomal protein S19 \\
\hline 6 & K03205 & type IV secretion system protein VirD4 \\
\hline 7 & K03294 & basic amino acid/polyamine antiporter, APA family \\
\hline 8 & K03496 & chromosome partitioning protein \\
\hline ID & Gene Pathway & \\
\hline 1 & HISDEG-PWY & L-histidine degradation I \\
\hline 2 & ILEUSYN-PWY & L-isoleucine biosynthesis I (from threonine) \\
\hline 3 & PWY-6122 & 5-aminoimidazole ribonucleotide biosynthesis II \\
\hline 4 & PWY-6595 & superpathway of guanosine nucleotides degradation (plants) \\
\hline 5 & PWY-6606 & guanosine nucleotides degradation II \\
\hline 6 & PWY-7111 & pyruvate fermentation to isobutanol (engineered) \\
\hline 7 & PWY-7219 & adenosine ribonucleotides de novo biosynthesis \\
\hline 8 & VALSYN-PWY & L-valine biosynthesis \\
\hline
\end{tabular}

D.

\begin{tabular}{|c|c|l|}
\hline \multicolumn{2}{|c|}{ ChAT-Cre Post 9 } \\
\hline ID & Gene Family & \multicolumn{1}{|c|}{ Annotation } \\
\hline 1 & K02003 & putative ABC transport system ATP-binding protein \\
\hline 2 & K02518 & translation initiation factor IF-1 \\
\hline 3 & K02913 & large subunit ribosomal protein L33 \\
\hline 4 & K02946 & small subunit ribosomal protein S10 \\
\hline 5 & K02948 & small subunit ribosomal protein S11 \\
\hline 6 & K02950 & small subunit ribosomal protein S12 \\
\hline 7 & K02965 & small subunit ribosomal protein S19 \\
\hline 8 & K03205 & type IV secretion system protein VirD4 \\
\hline ID & Gene Pathway & \\
\hline 1 & ILEUSYN-PWY & L-isoleucine biosynthesis I (from threonine) \\
\hline 2 & PWY-6151 & S-adenosyl-L-methionine cycle I \\
\hline 3 & PWY-6700 & queuosine biosynthesis I (de novo) \\
\hline 4 & PWY-6859 & all-trans-farnesol biosynthesis \\
\hline 5 & PWY-7111 & pyruvate fermentation to isobutanol \\
\hline 6 & PWY-7219 & adenosine ribonucleotides de novo biosynthesis \\
\hline 7 & PWY0-1296 & purine ribonucleosides degradation \\
\hline 8 & VALSYN-PWY & L-valine biosynthesis \\
\hline
\end{tabular}


Figure Supplement 5. ENS Activation-Mediated Changes in Feces (Related to Figure 5). LEfSe Cladograms for feces on (A) Day 9 of ChAT-Cre mice and (B) Day 5 of TH-Cre mice. Annotations are labelled in the tables below the cladogram $(C$ and $D)$ Microbial gene family and pathway annotations for the defining features labelled on the DEICODE PCoA plots in Figure 5O-5R. Colors in ID column refer to the color of feature arrows labelled in main figure. 
bioRxiv preprint doi: https://doi.org/10.1101/2021.04.12.439539; this version posted October 15, 2021. The copyright holder for this preprint (which was not certified by peer review) is the author/funder. All rights reserved. No reuse allowed without permission.

Figure 6

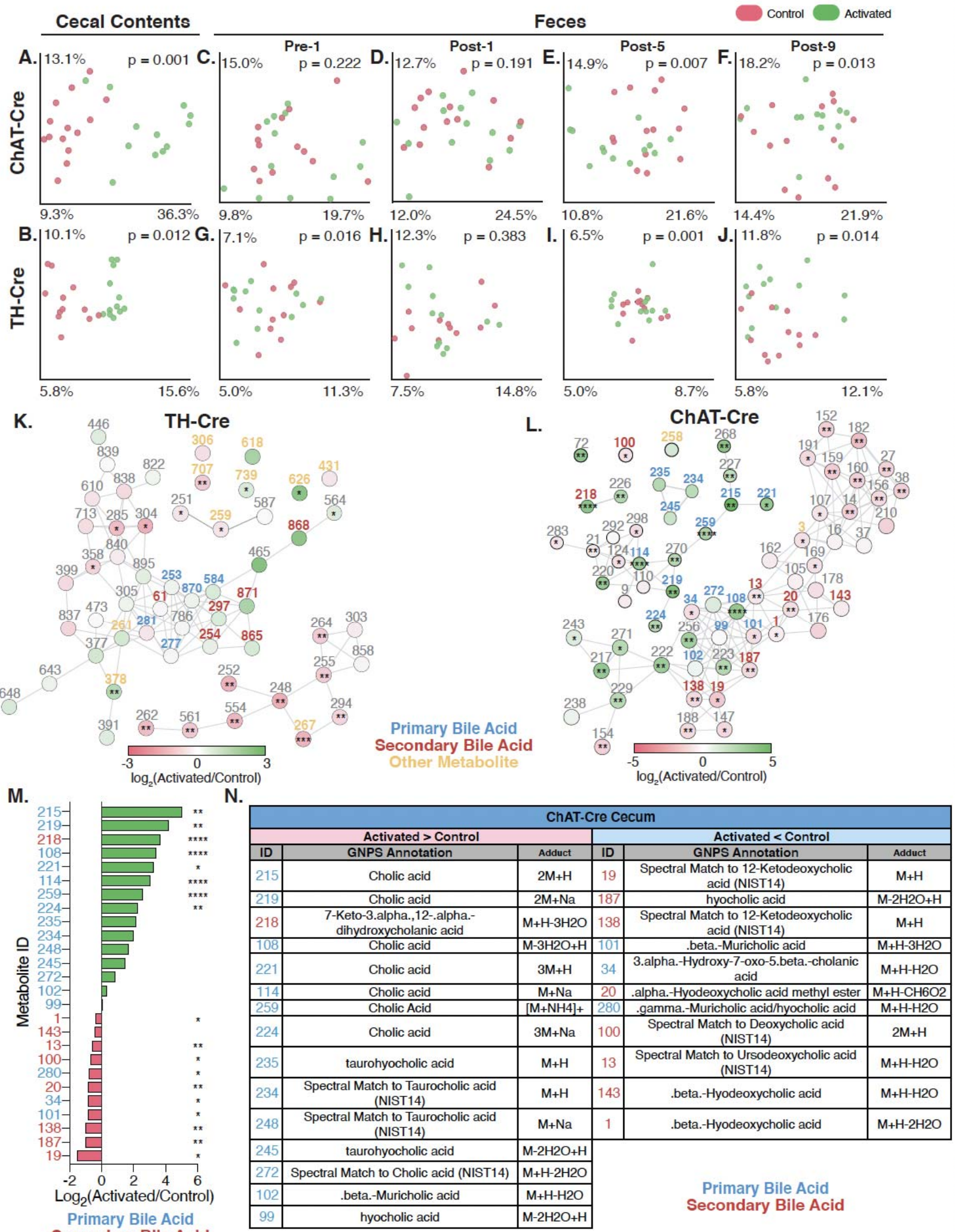


Figure 6. ENS Activation Alters the Host and Microbe-Derived Luminal Metabolites. (A) Canberra PoCA of the cell-free, luminal metabolome of control (red) and activated (green) cecal contents in ChAT-Cre and (B) TH-Cre mice. Statistical analyses were performed in QIIME2 as done previously (Bolyen et al., 2019) and axes for data visualization were chosen to minimize experimental biases (See Methods). (C-J) Fecal metabolome assayed at different timepoints before and after C21 administration in (C-F) ChAT-Cre and (G-J) TH-Cre mice. Axes for data visualization were chosen to minimize experimental biases (See Methods). (K) Metabolic networks constructed from identified cecal metabolites in TH-Cre and (L) ChAT-Cre mice. Each node is labelled with an ID number, corresponding to annotations, mass-to-charge ratios, retention times, fold changes, and significance values found in Table s2. Each node is colored by its upregulation (green) or downregulation (red) in activated groups. IDs in bold text have an annotation in GNPS and are colored by classes class of metabolite (primary bile acids, secondary bile acids, and other metabolites). (M) Fold-changes of specific bile acids identified to be upregulated (green bars) and downregulated (red bars) in activated ChAT-Cre mice. (N) Annotations of bile acids highlighted in (M). Metabolite IDs corresponding to annotated primary bile acids (blue type) and secondary bile acids (red type). Metabolite IDs are distinct by genotype ( $N=12-14$ for each group analyzed, $\left.{ }^{*}: p<0.05,{ }^{* *}: p<0.01,{ }^{* * *}: p<0.001,{ }^{* * *}: p<0.0001\right)$

Figure Supplement 6. PCoA of metabolic profiles comparing TH- and ChAT-Cre mice before and after neuronal activation.

Table Supplement 2. Extended annotations of networked metabolites related to Figure 6K-6N

https://github.com/mazmanianlab/Yoo et al 2021/blob/main/metabolomics/Table Supplement 2-Extended GNPS annotations of Metabolite Network Notes related to figure 6.xIsx

Source Data 6. Source data for Figure 6 and Figure Supplement 6

Figure 6M -

https://github.com/mazmanianlab/Yoo et al 2021/blob/main/metabolomics/fold changes of sp ecific bile acids.txt 
Figure Supplement 6

A.

Control - Post C21 - Cecum

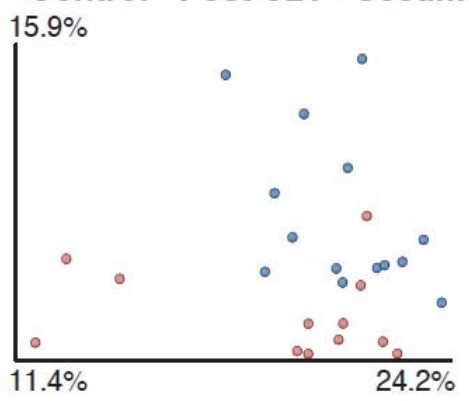

C. Control - Pre C21 - Feces
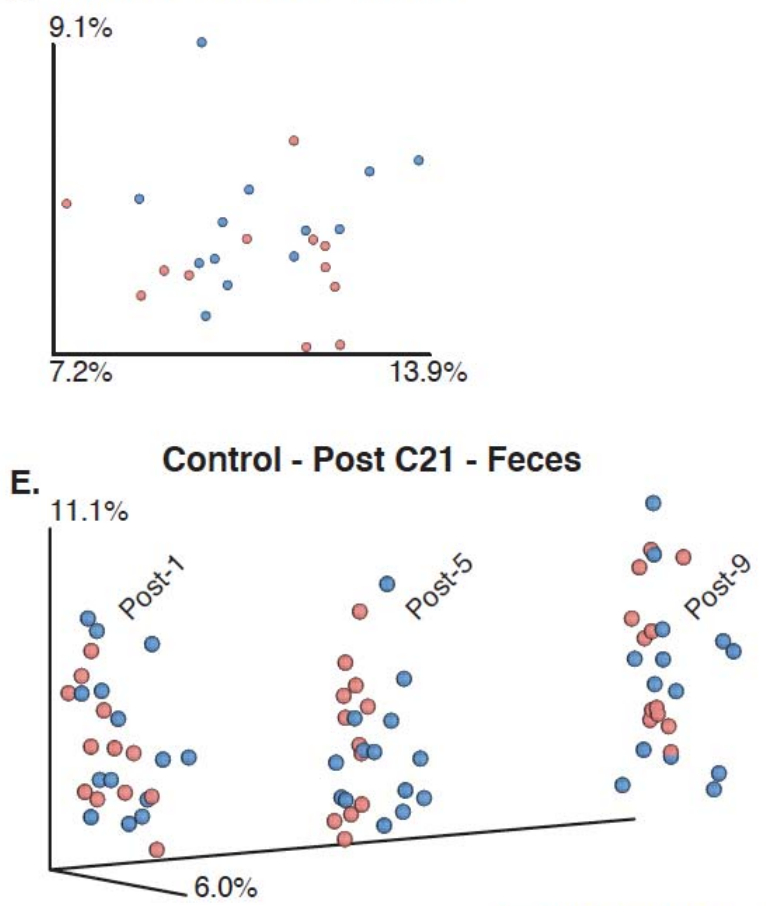

ChAT-Cre
B.

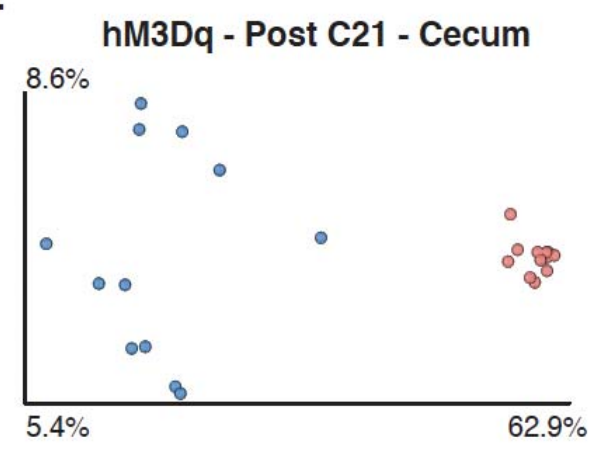

D. hM3Dq - Pre C21 - Feces

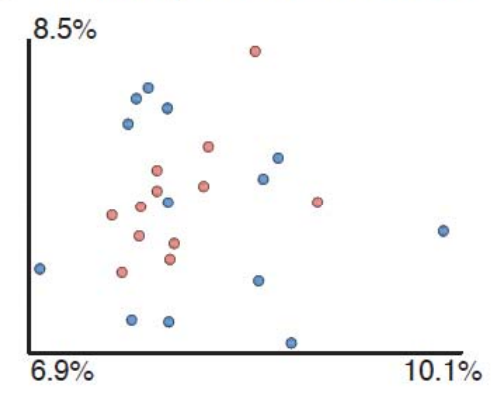

F. $9.4 \%$
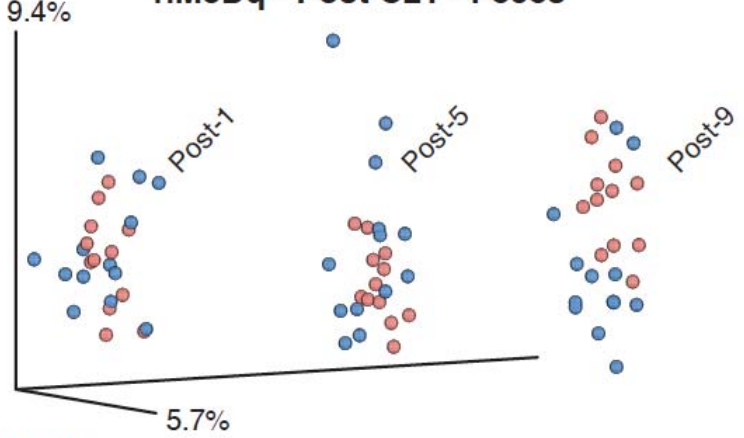

\section{TH-Cre}

Figure Supplement 6. Class Differences in ChAT-Cre vs. TH-Cre Metabolites Upon Activation (Related to Figure 6). Canberra PCoA analysis comparing activation-mediated changes to the $(\mathrm{A}-\mathrm{B})$ cecal and $(\mathrm{C}-\mathrm{F})$ fecal metabolome in ChAT-Cre versus TH-Cre mice (A) Cecal metabolome of control mice (B) Cecal metabolome of activated mice (C) Pre-C21 feces of control and (D) activated mice (E) Fecal metabolome during activation time course for control and $(F)$ activated mice. 


\section{Table Supplement Legends}

Table Supplement 1 - Gene Set Enrichment Analysis of Gene Ontology (GO) Terms (Related to Figure 3).

Gene set enrichment analysis of gene ontology (GO) terms that are upregulated in the distal SI and proximal colon of activated ChAT- and TH-Cre mice.

Table Supplement 2 - Extended GNPS annotations of Metabolite Network Nodes (Related to Figure 6).

Extended annotations for networked (bold), MS/MS spectra in cecal luminal contentscorresponds to Figure 6K and 6L in ChAT-Cre and TH-Cre mice, respectively. 


\section{METHODS}

\section{Mice}

All mouse experiments were performed in accordance with the NIH Guide for the Care and Use of Laboratory Animals using protocols approved by the Institutional Animal Care and Use Committee at the California Institute of Technology. TH-Cre (from Viviana Gradinaru at Caltech. Originally from Ted Ebendal B6.129X1-Thtm1(cre)Te/Kieg) (Lindeberg et al., 2004) and ChATCre mice (Jackson Laboratories, Bar Harbor, ME- Stock\# 028861) were obtained from Jackson Laboratories, and homozygous Cre-mice were bred to wild-type mice to yield male and female heterozygous Cre-mice used for our studies. Wildtype specific pathogen free (SPF), C57BL/6 (Jackson Laboratories, Bar Harbor, ME Stock \#000664) males and females were used for breeding and experiments.

\section{Virus Production}

Virus was produced using methods described in Challis et. al. (Challis et al., 2019) Briefly, human embryonic kidney (HEK293T) cells were triple-transfected with pUCmini-iCAP-AAVPHP.S, pHelper Plasmid, and one of the following pAAV genomes (CAG-NLS-GFP, hSYN1tDTomato, GFAP-tDTomato, hSYN1-mRuby2, hSYN1-DiO-mRuby2, hSYN1-mNeonGreen, hSYN1-DiO-mNeonGreen, hSYN1-mTurquoise2, hSYN1-DiO-mTurquoise2, hSYN1-DiOhM3Dq-mRuby2, CAG-GCaMP6F). Cells were grown in DMEM + Glutamax + Pyruvate (Gibco, Gaithersburg, MD- Stock\# 10569-010) + 5\% FBS + non-essential amino acids (Gibco, Gaithersburg, MD- Stock\# 11140-050), penicillin-streptomycin (Gibco, Gaithersburg, MDStock\# 15070-063). Virus is precipitated from cells and supernatant with an 8\% PEG solution (wt/vol), and purified by ultracentrifugation using 15\%, 25\%, 40\%,60\% stacked iodixanol gradients.

\section{Systemic Delivery of AAV- Titers}

Mice were anesthetized using $2 \%$ isoflurane. Virus was titered to $10^{12} \mathrm{VGs}$, resuspended to a volume of $100 \mu \mathrm{l}$ with sterile PBS, and injected retro-orbitally.

\section{Quantitative PCR of Viral Abundance}

AAV-PHP.S:hSYN1-mNeonGreen was delivered systemically to WT mice at 6-8 weeks of age. 3-4 weeks following viral infection, $1 \mathrm{~cm}$ of proximal, medial, and distal SI and colon were harvested and flash frozen in TRIzol (ThermoFisher Scientific, Waltham, MA- Cat. No. 15596018) and RNA was extracted per manufacturer's instructions. Viral ssDNA is present in the RNA fraction. Contaminating RNA was eliminated with RNase treatment (ThermoFisher Scientific, Waltham, MA- Cat. No. AM228) per manufacturer's instructions. Quantitative PCR was performed on viral DNA with primers against WPRE (Forward: 5'-

GGCTGTTGGGCACTGACAAT-3'; Reverse: 5'-CCGAAGGGACGTAGCAGAAG-3') and values were normalized to ubiquitous mitochondrial gene, mtRNR2 (Forward: 5'-

CCGCAAGGGAAAGATGAAAA-3'; Reverse: 5'-TCGTTTGGTTTCGGGGTTTC-3'). Plotted relative abundances are mean values across three regions sampled in either SI or colon.

\section{Neural activation of the GI tract}

TH-Cre and ChAT-Cre mice are used for these experiments. "Activated" mice are infected with AAV-PHP.S:hSYN1-DIO-hM3Dq-mRuby2 and "Control Mice" are infected with AAVPHP.S:hSYN1-DIO-mRuby2. This is to control for both AAV-PHP.S-mediated expression and the effects of Compound 21 dihydrochloride (C21) (HelloBio, Princeton, NJ- HB6124). C21 was injected intraperitoneally at a dose of $3 \mathrm{mg} / \mathrm{kg}$ in both activated and control mice. Mice for time course experiments were single-housed in sterile cages with autoclaved water following first 
C21 administration (C21 administered for 10 consecutive days). Injections of C21 were administered at the same time every day (10AM).

Tissue preparation, immunohistochemistry, Imaging, and Quantification

$100 \mathrm{mg} / \mathrm{kg}$ of pentobarbital (Euthasol - Virbac, Carros, France) was administered IP, and tissues were perfused with $30 \mathrm{~mL}$ of phosphate buffer solution (PBS) and next with cold $4 \%$ paraformaldehyde (PFA) in PBS. Gl tract was post-fixed in $4 \%$ PFA overnight at $4{ }^{\circ} \mathrm{C}$, and stored in PBS $+0.025 \%$ sodium azide. Tissues that underwent subsequent immunohistochemistry were made transparent by use of the passive CLARITY technique (PACT) (Treweek et al., 2015). Briefly, perfused and fixed tissues were embedded with polymerized $4 \%$ (wt/vol) acrylamide, and lipids were eliminated using $8 \%$ (wt/vol) SDS solution. Tissues were blocked in $3 \%$ donkey serum and permeabilized with PBS $+0.3 \%$ Triton (PBST). Primary antibodies were incubated in PBST for 48 hours and washed with PBST for 24 hours (replacing wash 3 times). Tissues were next incubated in secondary antibodies (and DAPI) for 24 hours and washed in PBS for 48 hours, intermittently replacing the wash solution with fresh PBS. Primary antibodies used were rabbit PGP9.5 (1:300; Millipore Sigma AB1761-I) and chicken GFAP (1:500, BioLegend Cat \#829401). Secondary antibodies used were goat anti-chicken Alexa 647 (Life Technologies A-21450) and donkey anti-rabbit Alexa 568 (Life Technologies A10042). Tissues imaged just for virally expressed, endogenous fluorescence were made transparent using sorbitol-based optical clearing method, ScaleS (Hama et al., 2015). Tissues were mounted in respective mounting mediums (RIMS and Scales S4), on a glass slide with a $0.5 \mathrm{~mm}$ spacer (iSpacer, SunJin Lab Co.). Images were acquired on Zeiss LSM 780 or 880, and microscope, laser settings, contrast, and gamma remained constant across images that were directly compared. All confocal images were taken with the following objectives: Fluar $5 \times 0.25 \mathrm{M} 27$ Plan-Apochromat 10× 0.45 M27 (work- ing distance $2.0 \mathrm{~mm}$ ) and Plan-Apochromat $25 \times 0.8$ Imm Corr DIC M27 multi- immersion. Neurons in each ganglion were counted by counting cells that are of a distinct color. Large intestinal ganglia were defined as distinct if separated by a width of 3 or fewer neurons.

\section{Live, in vivo, imaging (GCaMP6F), Video Processing, and Analysis}

AAV-PHP.S-CAG-GCaMP6F (10 ${ }^{12}$ VGs) was delivered systemically to WT C57. 3-4 weeks after infection, mice were anesthetized with $2 \%$ isoflurane on a heating pad (Kent Scientific, Torrington, CT- DCT15) with plastic sleeve covers (Kent Scientific, Torrington, CT- DCT1520P). The abdominal cavity was surgically opened to expose the intestines. The proximal colon was identified, and this portion was placed on top of a stack of 4-6 glass microscopy slides (depending on size of animal) (VWR, Radnor PA- Cat. No. 48300-026). Tissue was secured onto glass slide with a biorthogonal silicon elastomer (Kwik-Sil) (World Precision Instruments, Sarasota, FL- KWIK-SIL), and a glass coverslip. Elastomer stiffens within 1 minute of application and coverslip must be held steadily to ensure a flat imaging surface. Anesthetized mouse is placed under an upright confocal microscope (Zeiss LSM 880). Using a 10X objective, GCaMP6F fluorescence was taken at $5 \mathrm{~Hz}$ (1 image every $200 \mathrm{~ms}$ ). Periods of movement of tissue and luminal contents is normal during live imaging. Cell tracking was performed in 2D by using TrackMate ImageJ plugin (https://imagej.net/TrackMate) and fluorescence intensity was recorded from cells. Average background was determined by taking the fluorescence of a region of interest that did not contain a cell, over the duration of the video. Background was subtracted from cell fluorescence intensities.

\section{Spike Detection}

Data sets of fluorescent values recorded at a rate of 0.206 s from GCaMP6F-expressing unstimulated neurons in the myenteric plexus of the proximal large intestine and analyzed with the MLspike software for Matlab downloaded from GitHub (https://github.com/MLspike/spikes) 
(Deneux et al., 2016). MLspike determines a new baseline to subtract from the raw fluorescence data to allow accurate modelling. The software uses a version of the Viterbi algorithm to obtain the most probable spike train. From the model, fluctuating baseline, model-estimated spike train, and most probable spike times were extracted. Polynomial coefficients $\left(p_{2}\right)$ were changed from 0.5 to 0.55 and $\left(p_{3}\right)$ from 0.01 to 0.03 for GCaMP6F fluorescence as recommended (Deneux et al., 2016). The minimum range for baseline $\left(b_{\min }\right)$ was changed from 0.7 to 0.5 as determined by observed fluorescence values.

\section{GCaMP6F Fluorescence in Ex Vivo Intestinal Preparation}

Small intestinal tissue was quickly harvested from ChAT-Cre mice, flushed and placed in oxygenated (95\% O2, 5\% CO2), ice cold Krebs-Henseleit solution for 1 hour followed by $15 \mathrm{~min}$ at room temperature. A segment was cut along the mesenteric attachment, and pinned flat (mucosa facing down) on a Sylgard-lined recording chamber (Warner Instruments, PH1) in oxygenated Krebs-Henseleit solution. C21 was added at 10nM and GCaMP6F fluorescence was detected on an upright microscope (Zeiss, Oberkochen, Germany- Examiner D1).

\section{3' mRNA-sequencing}

\section{Tissue collection and RNA extraction}

Mice were cervically dislocated and the Gl tract was removed. $1 \mathrm{~cm}$ of tissue above and below the cecum were dissected and cleaned to represent tissue from the distal SI and proximal colon, respectively. Tissue was homogenized in TRIzol (ThermoFisher Scientific, Waltham. MA- Cat. No. 15596018) solution using bead-based homogenizing methods, and total RNA was extracted using chloroform per manufacturer's instructions.

\section{Library Preparation, Sequencing, and Analysis}

The cDNA libraries were prepared using the QuantSeq 3'mRNA-Seq Library Prep Kit FWD for Illumina (Lexogen, Greenland, NH) supplemented with UMI (unique molecular index) as per the manufacturer's instructions. Briefly, total RNA was reverse transcribed using oligo (dT) primers. The second cDNA strand was synthesized by random priming, in a manner that DNA polymerase is efficiently stopped when reaching the next hybridized random primer, so only the fragment closed to the 3' end gets captured for later indexed adapter ligation and PCR amplification. UMls were incorporated to the first 6 bases of each read, followed by 4 bases of spacer sequences. UMls are used to eliminate possible PCR duplicates in sequencing datasets and therefore facilitate unbiased gene expression profiling. The basic principle behind the UMI deduplication step is to collapse reads with identical mapping coordinates and UMI sequences. This step helps increase the accuracy of sequencing read counts for downstream analysis of gene expression levels. The processed libraries were assessed for its size distribution and concentration using Bioanalyzer High Sensitivity DNA Kit (Agilent Technologies, Santa Clara, CA- Cat. No. 5067-4626 and -4627). Pooled libraries were diluted to 2 nM in EB buffer (Qiagen, Hilden, Germany, Cat. No. 19086) and then denatured using the Illumina protocol. The libraries were pooled and diluted to $2 \mathrm{nM}$ using $10 \mathrm{mM}$ Tris- $\mathrm{HCl}, \mathrm{pH} 8.5$ and then denatured using the Illumina protocol. The denatured libraries were diluted to $10 \mathrm{pM}$ by pre-chilled hybridization buffer and loaded onto an Illumina MiSeq v3 flow cell for 150 cycles using a single-read recipe according to the manufacturer's instructions. Single-end 75bp reads (max 4.5M reads) were obtained. De-multiplexed sequencing reads were generated using Illumina BaseSpace.

UMI specific workflows that were developed and distributed by Lexogen were used to extract reads that are free from PCR artifacts (i.e., deduplication). First, the umi2index tool was used to add the 6 nucleotide UMI sequence to the identifier of each read and trims the UMI from the start of each read. This generates a new FASTQ file, which is then processed through trimming and alignment. Second, after the quality and polyA trimming by BBDuk (https://jgi.doe.gov/dataand-tools/bbtools/bb-tools-user-guide/bbduk-guide/and alignment by HISAT2 (version 2.1.0) 
(Kim et al., 2015), the mapped reads are collapsed according to the UMI sequence of each read. Reads are collapsed if they have the same mapping coordinates (CIGAR string) and identical UMI sequences. Collapsing reads in this manner removes PCR duplicates. Read counts were calculated using HTSeq (Anders et al., 2015) by supplementing Ensembl gene annotation (GRCm38.78). Raw read counts were run through ShinySeq to obtain differentially expressed genes and downstream gene ontology analyses (Sundararajan et al., 2019).

\section{Proteome Preparation Protein extraction}

Mice were sacrificed 45 minutes after C21 administration and cecal contents were isolated and resuspended in $400 \mu \mathrm{l}$ of phosphate buffered solution, and centrifuged at $\times 20,000 \mathrm{~g}$ to spin down cells and lysate. Protein was isolated from resulting supernatant using Wessel-Flüegge's methanol/chloroform extraction method (Wessel and Flügge, 1984). Briefly, MeOH and chloroform was added to sample at a $4: 1$ and $1: 1$ ratio, respectively. Next, water $\mathrm{dH} 2 \mathrm{O}$ was added at a 3:1 ratio, samples were vortexed and centrifuged at x20,000g. Resulting precipitated protein was collected and washed with methanol. Precipitated protein was centrifuged and was left to air dry, and stored in -20C until protein digestion.

\section{In-solution Protein Digestion and Desalting}

Precipitated protein sample was denatured in $40 \mu \mathrm{l}$ of $8 \mathrm{M}$ Urea (100mM Tris- $\mathrm{HCl} \mathrm{pH} 8.5)$. To reduce disulfide bonds, $1.25 \mu$ of $100 \mathrm{mM}$ Tris(2-carboxyethyl)Phosphine was added and incubated at room temperature (RT) for 20 minutes. Then $1.8 \mu \mathrm{l}$ of $250 \mathrm{mM}$ iodoactaminde was added and incubated at RT in the dark to alkylate cysteines. The first step of digestion was initiated by adding $1 \mu \mathrm{l}$ of $0.1 \mu \mathrm{g} / \mu \mathrm{l}$ of Lysyl endopeptidase. After four-hour incubation. Urea concentration was adjusted to $2 \mathrm{M}$ by adding $120 \mu \mathrm{l}$ of $100 \mathrm{mM}$ Tris- $\mathrm{HCl} \mathrm{pH} 8.5$. The second step of digestion was initiated by adding $2.5 \mu \mathrm{l}$ of $2 \mu \mathrm{g} / \mu \mathrm{l}$ trypsin plus $1.6 \mu \mathrm{l}$ of $100 \mathrm{mM} \mathrm{CaCl} 2$ for trypsin activity enhancement and incubating overnight in the dark. Formic acid was added to stop trypsing digestion. The digested peptides were desalted by HPLC using C8 peptide microtrap (Microm Bioresources). Peptides were lyophilized and diluted to $200 \mathrm{ng} / \mu \mathrm{l}$ in $0.2 \%$ formic acid prior to LC-MS/MS analysis.

\section{LC-MS/MS}

Samples were analyzed on a Q Exactive HF Orbitrap mass spectrometer coupled to an EASY nLC 1200 liquid chromatographic system (Thermo Scientific, San Jose, CA). Approximately 200 ng of peptides were loaded on a $50 \mu \mathrm{m}$ I.D. $\times 25 \mathrm{~cm}$ column with a $10 \mu \mathrm{m}$ electrospray tip (PicoFrit from New Objective, Woburn, MA) in-house-packed with ReproSil-Pur C18-AQ 1.9 $\mu \mathrm{m}$ (Dr. Maisch, Ammerbuch, Germany). Solvent A consisted of $2 \% \mathrm{MeCN}$ in $0.2 \% \mathrm{FA}$ and solvent $\mathrm{B}$ consisted of $80 \% \mathrm{MeCN}$ in $0.2 \% \mathrm{FA}$. A non-linear 60 minute gradient from $2 \% \mathrm{~B}$ to $40 \% \mathrm{~B}$ was used to separate the peptides for analysis. The mass spectrometer was operated in a datadependent mode, with MS1 scans collected from 400-1650 m/z at 60,000 resolution and MS/MS scans collected from $200-2000 \mathrm{~m} / \mathrm{z}$ at 30,000 resolution. Dynamic exclusion of $45 \mathrm{~s}$ was used. The top 12 most abundant peptides with charge states between 2 and 5 were selected for fragmentation with normalized collision energy of 28.

\section{Peptide and Protein Identification}

Thermo .raw files were converted to .ms1 and .ms2 files using RawConverter 1.1.0.18 (He et al., 2015) operating in data dependent mode and selecting for monoisotopic $\mathrm{m} / \mathrm{z}$. Tandem mass spectra (.ms2 files) were identified by database search method using the Integrated Proteomics Pipeline 6.5.4 (IP2, Integrated Proteomics Applications, Inc., http://www.integratedproteomics.com). Briefly, databases containing forward and reverse (decoy) (Elias and Gygi, 2007; Peng et al., 2003) peptide sequences were generated from in silico trypsin digestion of either the mouse proteome (UniProt; Oct. 2, 2019) or protein sequences derived from large comprehensive public repositories (ComPIL 2.0) (Park et al., 
2018). Tandem mass specta were matched to peptide sequences using the ProLuCID/SEQUEST (1.4) (Xu et al., 2015; 2006) software package. The validity of spectrumpeptide matches were assessed using the SEQUEST-defined parameters XCorr (crosscorrelation score) and DeltaCN (normalized difference in cross-correlation scores) in the DTASelect2 (2.1.4) (Cociorva et al., 2007; Tabb et al., 2002) software package. Search settings were configured as follows: (1) 5ppm precursor ion mass tolerance, (2) 10ppm fragment ion mass tolerance, (3) $1 \%$ peptide false discovery rate, (4) 2 peptide per protein minimum, (5) 6006000Da precursor mass window, (6) 2 differential modifications per peptide maximum (methionine oxidation: M+15.994915 Da), (7) unlimited static modications per peptide (cysteine carbamidomethylation: $\mathrm{C}+57.02146 \mathrm{Da}$ ), and (8) the search space included half- and fully tryptic (cleavage $\mathrm{C}$-terminal to $\mathrm{K}$ and $\mathrm{R}$ residues) peptide candidates with unlimited (mouse database, custom metagenomic shotgun database) or 2 missed cleavage events (ComPIL 2.0).

\section{Differential Analysis of Detected Proteins using Peptide-Spectrum Matches (Spectral Counts)}

Detected proteins were grouped by sequence similarity into "clusters" using CD-HIT 4.8 .1 (Fu et al., 2012; Li and Godzik, 2006; Li et al., 2001) at the following similarity cut-offs: $65 \%, 75 \%$, $85 \%$, and $95 \%$. The following is an example command line input: "cd-hit -i fastafile.fasta -0 outputfile -c 0.65 -g 1 -d 0". Tandem mass spectra identified as peptides (peptide spectrum matches, PSMs) were mapped to CD-HIT generated clusters. PSMs mapping to $>1$ cluster were discarded. Cluster-PSM tables were generated and differential analysis was performed in DESeq2 (1.25.13) (Love et al., 2014). Briefly, count data (PSMs) were modeled using the negative binomial distribution, and the mean-variance relationship was estimated. Variance was estimated using an information sharing approach whereby a single feature's (or cluster's) variance was estimated by taking into account variances of other clusters measured in the same experiment. Feature significance calling and ranking were performed using estimated effect sizes. Multiple testing correction was performed by Benjamini-Hochberg method within the DESeq2 package. Volcano plots were generated in Prism (GraphPad).

\section{Differential Analysis of Detected Proteins using Ion Intensity (Precursor Intensity)}

Detected proteins were grouped into "clusters" by sequence similarity using CD-HIT 4.8.1 (Fu et al., 2012; Li and Godzik, 2006; Li et al., 2001) at the following similarity cut-offs: $65 \%, 75 \%$, $85 \%$, and $95 \%$. The following is an example command line input: "cd-hit -i fastafile.fasta -o outputfile -c 0.65 -g 1 -d 0". Using the Census software package (Park et al.; 2008) (Integrated Proteomics Pipeline 6.5.4), peptide ion intensities were calculated from .ms1 files. Peptide ion intensities were assigned to their parent peptide, then parent peptides were mapped to their appropriate CD-HIT generated clusters. Ion intensities belonging to parent peptides that map to $>1$ CD-HIT cluster were discarded. Cluster-ion intensity tables were generated.

Ion intensity data were analyzed using the DEP package (Zhang et al., 2018) operating in R. Intensity values were automatically Log2 transformed in DEP. The cluster list was subsequently filtered with the 'filter_proteins' function such that clusters with missing values above a $65 \%$ threshold were discarded. Remaining intensities were further transformed by the 'normalize_vsn' function (Huber et al., 2002). Missing data in remaining clusters were imputed using a mixed approach. Clusters where either the control or treatment group contained only null entries were classified as 'missing not at random' (MNAR) and imputed with 0 values. All other groups were treated as 'missing at random' (MAR) and imputed using the maximum likelihood method ('MLE') (Gatto and Lilley, 2012). Note that for a given cluster, missing values for treatment groups were imputed separately by treatment group. Differential expression analyses were performed on filled-in cluster-ion intensity tables using the 'test_diff' function (Ritchie et al., 2015) and multiple testing correction was performed using the 'add_rejections' function.

\section{Network Analysis using STRING Database}


Upregulated proteins with a nominal $p$-value $<0.2$ were searched against protein-protein interactions in the STRING database (http://www.string-db.org) where high confidence interactions were selected for. Briefly, the STRING database sources protein-protein interactions from primary databases consisting of genomic context predictions, high-throughput lab experiments, (conserved) co-expression, automated textmining, and pervious knowledge in databases (Szklarczyk et al., 2019).

\section{Metaproteome Analysis using Unipept}

Upregulated tryptic, microbial peptide sequences, with fold change and nominal p-value cutoffs of 2 and $p<0.2$ respectively, were input into Unipept (http://unipept.ugent.be) (Gurdeep Singh et al., 2019; Mesuere et al., 2015), equating leucine and isoleucine and filtering duplicate peptides. Briefly, Unipept indexes tryptic peptide sequences from the UniProtKB and details peptides with NCBl's taxonomic database. Lowest common ancestor is calculated for each tryptic peptide.

\section{Metagenome Preparations Fecal Collection}

AAV-PHP.S:hSYN1-hM3Dq-mRuby2 (10 12 VGs) was delivered systemically to TH-Cre and ChAT-Cre mice. 3-4 week after infection, C21 (3mg/kg) was administered daily for 10 consecutive days. Fecal pellets were collected in sterile containers one day before initial C21 dose, and between doses thereafter.

\section{Fecal sample DNA extraction and Library Preparation}

DNA was extracted with the Qiagen MagAttract PowerSoil DNA kit as previously described (Marotz et al 2017). Our standard protocol is optimized for an input quantity of $1 \square$ ng DNA per reaction. Prior to library preparation, input DNA is transferred to a 384-well plate and quantified using a PicoGreen fluorescence assay (ThermoFisher, Inc). Input DNA is then normalized to $1 \square \mathrm{ng}$ in a volume of $3.5 \square \mu \mathrm{L}$ of molecular-grade water using an Echo 550 acoustic liquidhandling robot (Labcyte, Inc). Enzyme mixes for fragmentation, end repair and A-tailing, ligation, and PCR are prepared and added in approximately 1:8 scale volumes using a Mosquito HV micropipetting robot (TTP Labtech). Fragmentation is performed at $37 \square^{\circ} \mathrm{C}$ for $20 \square \mathrm{min}$, followed by end repair and A-tailing at $65 \square{ }^{\circ} \mathrm{C}$ for $30 \square \min$.

Sequencing adapters and barcode indices are added in two steps, following the iTru adapter protocol (Glenn et al., 2019). Universal adapter "stub" adapter molecules and ligase mix are first added to the end-repaired DNA using the Mosquito HV robot and ligation performed at $20 \square^{\circ} \mathrm{C}$ for $1 \square \mathrm{h}$. Unligated adapters and adapter dimers are then removed using AMPure XP magnetic beads and a BlueCat purification robot (BlueCat Bio). $7.5 \mu \mathrm{L}$ magnetic bead solution is added to the total adapter-ligated sample volume, washed twice with $70 \% \mathrm{EtOH}$, and then resuspended in 7 $\square \mathrm{L}$ molecular-grade water.

Next, individual i7 and i5 are added to the adapter-ligated samples using the Echo 550 robot. Because this liquid handler individually addresses wells, and we use the full set of 384 unique error-correcting i7 and i5 indices, we are able to generate each plate of 384 libraries without repeating any barcodes, eliminating the problem of sequence misassignment due to barcode swapping (Costello et al., 2018; Sinha et al., 2017). To ensure that libraries generated on different plates can be pooled if necessary, and to safeguard against the possibility of contamination due to sample carryover between runs, we also iterate the assignment of i7 to i5 indices each run, such that each unique i7:i5 index combination is only repeated once every 147,456 libraries. $4.5 \square \mu \mathrm{L}$ of eluted bead-washed ligated samples is added to $5.5 \square \mu \mathrm{L}$ of PCR master mix and PCR-amplified for $15 \square$ cycles. The amplified and indexed libraries are then purified again using magnetic beads and the BlueCat robot, resuspended in $10 \square \mu \mathrm{L}$ water, and $9 \square \mu \mathrm{L}$ of final purified library transferred to a 384-well plate using the Mosquito HV liquid- 
handling robot for library quantitation, sequencing, and storage. 384 samples are then normalized based on a PicoGreen fluorescence assay.

\section{Shallow shotgun metagenome sequencing and diversity analysis}

The Illumina data for each HiSeq lane was uploaded to Qiita, a tool with standardized pipelines for processing and analyzing metagenomic data (Gonzalez et al., 2018). Adapter sequences were removed from the reads using the Atropos v.1.1.15 (Didion et al., 2017) command (from the qp-shogun 0.1.5 pipeline) and the trimmed sequences were downloaded from Qiita. The reads for each sample were filtered of any potential mouse contamination using Bowtie2 v.22.2.3 (Langmead and Salzberg, 2012). The filtered reads were then aligned to the Web of Life (WoL) reference phylogeny (Zhu et al., 2019) with Bowtie2 using an adapted SHOGUN pipeline (Hillmann et al., 2018). The WoL contains 10,575 bacterial and archaeal genomes with each genome representing an OTU. Sequencing reads that did not map to a single reference genome as well as reads that mapped to multiple genomes were not included in the analysis. If an OTU had a relative abundance less than $0.01 \%$ in a given sample, the OTU was not included for that sample. Additionally, OTUs with fewer than 5 assigned reads were not considered. The samples were rarefied to a depth of 12,750 reads and those with fewer than the rarefaction depth were excluded. The QIIME2 v.2019.7 (Bolyen et al., 2019) DEICODE plugin was used to calculate the Aitchison distances, a compositional beta diversity metric, and perform Robust Aitchison PCA to create biplots that visualize relationships between features and samples (Martino et al., 2019). The QIIME2 diversity plugin was used to calculate the other alpha- and beta-diversity metrics used in this study.

\section{Metagenomic based functional profiling}

The filtered reads were also analyzed using HUMAnN2 v2.8.1 (Franzosa et al., 2018) to establish functional profiles for the samples. HUMAnN2 is a pipeline that begins by using MetaPhIAn 2 to compile custom databases of reference genomes based on the species detected in a sample (Truong et al., 2015). HUMAnN2 then maps the filtered onto these custom databases and the reads that do not map to any of the references are then subjected to a translated search against UniProt Reference Clusters or UniRef (Suzek et al., 2007). Here, the UniRef90 database was used for the translated search and installed according to the HUMAnN2 documentation. The results from both the search performed using the custom reference genome database and the search against the UniRef90 database were combined and the gene families identified in each sample were reported in units of read per kilobase (RPKs) to account for gene length. HUMAnN2 also compared the gene families found in a sample with the MetaCyc pathways database (Caspi et al., 2018) and output a table reporting the pathway abundances found in each sample. After rarefying gene family tables to a depth of 166,000 RPKs and using a depth of 22,600 for pathway abundances, the QIIME2 diversity and DEICODE plugins were used to calculate alpha- and beta-diversity metrics.

\section{Metabolomics Preparations Sample Preparation}

Frozen fecal and cecal samples were transported on dry ice for metabolomics analysis. The samples were weighed out and an extraction solvent (1:1 methanol to water with an internal standard of $1 \mu \mathrm{M}$ sulfamethazine) was added at a 1:10 milligram to microliter ratio. The samples were then homogenized using a TissueLyser II (Qiagen) for 5 minutes at 25 hertz followed by a 15 minute centrifugation at $14,000 \mathrm{rpm}$. From the supernatant, $120 \mu \mathrm{L}$ were transferred to a 96 deepwell plate (Eppendorf) and then these samples were lyophilized using a CentriVap Benchtop Vacuum Concentrator (Labconco) and stored at $-80^{\circ} \mathrm{C}$. Upon the time for data acquisition, the lyophilized plates were resuspended in a 1:1 methanol to water solvent spiked with $1 \mu \mathrm{M}$ of sulfadimethoxine. The plates were vortexed for 2 minutes, centrifuged at 14,000 
rpm for 15 minutes and $120 \mu \mathrm{L}$ of the supernatant was transferred to a 96 well autosampler plate(Eppendorf). Plates were stored at $4^{\circ} \mathrm{C}$ prior to LCMS analysis.

\section{Data Acquisition}

The untargeted metabolomics analysis was completed using an ultra-high performance liquid chromatography system (Thermo Dionex Ultimate 3000 UHPLC) coupled to ultra-high resolution quadrupole time of flight (qTOF) mass spectrometer (Bruker Daltonics MaXis HD). A Phenomenex Kinetex column (C18 $1.7 \mu \mathrm{m}, 2.1 \mathrm{~mm}$ x $50 \mathrm{~mm}$ ) was used for chromatographic separation. An injection volume of $5 \mu \mathrm{L}$ was used per sample and a flow-rate of $0.500 \mathrm{~mL}$ was used throughout the analysis. The mobile phase consisted of solvent A: $100 \%$ LC-MS grade water spiked with $0.1 \%$ formic acid and solvent B: $100 \%$ LC-MS grade acetonitrile spiked with $0.1 \%$ formic acid. The chromatographic gradient was as follows: $0.0-1.0 \square \mathrm{min}, 5 \% \mathrm{~B} ; 1.0-$ 9.0 $\square \min , 5-100 \%$ B; 9.0-11.0 $\square \min , 100 \%$ B; $11.0-11.5 \square \min , 100-5 \%$ B; $11.5-12.5$ min, 5\% B. The data was collected using electrospray ionization in positive mode. Sample data was saved as .d file folders.

\section{Data Processing}

The raw .d data files were converted to $\mathrm{mzXML}$ format using Bruker Compass DataAnalysis 4.1 software. The resulting .mzXML file, the original .d file folders, and basic prep information sheet were stored on the UC San Diego MassIVE data repository under the accession number MSV000084550. For the MS1 level feature detection, the open-source software MZmine version 2.51 was used. The parameters used are as follows: 1) Mass Detection (Centroid, Noise Level MS1 1E3, MS2 1E2); 2) ADAP Chromatogram Builder (Min Group size in \# of scans=3, Group Intensity Threshold=3E3, Min Highest Intensity=1E3, m/z tolerance $0.01 \mathrm{~m} / \mathrm{z}$ or $10.0 \mathrm{ppm}$ ); 3) Chromatogram Deconvolution (Local Minimum Search>Chromatographic Threshold 0.01\%, Minimum in RT range 0.50 min, <Minimum Relative Height $0.01 \%$, Minimum Absolute Height 3E3, Min Ratio of Peak Top/Edge 2, Peak Duration Range 0.05-0.50 min; m/z Calculation Auto, $\mathrm{m} / \mathrm{z}$ range for MS2 pairing 0.01 Da, and RT Range for MS2 Pairing $0.1 \mathrm{~min}$ ); Isotopic Peaks Grouper (m/z Tolerance $0.01 \mathrm{~m} / \mathrm{z}$ or $10.0 \mathrm{ppm}$, Retention Time Tolerance $0.3 \mathrm{~min}$, Maximum Charge 4, Representative Ion Most Intense); Join Aligner (m/z Tolerance $0.01 \mathrm{~m} / \mathrm{z}$ or 10.0 ppm, Weight for m/z 75, Retention Time Tolerance $0.3 \mathrm{~min}$, Weight for RT 25); Gap-Filling Peak Finder (Intensity Tolerance $20 \%, \mathrm{~m} / \mathrm{z}$ Tolerance $0.005 \mathrm{~m} / \mathrm{z}$ or $10.0 \mathrm{ppm}$, Retention Time Tolerance $0.2 \mathrm{~min})$. The resulting feature table was saved as a .csv file and. $\mathrm{mgf}$ file for use in GNPS and MetaboAnalyst.

\section{Molecular Networking and Statistical Analysis}

Molecular networking was performing using the feature networking tool available on the Global Natural Products Social Molecular Networking portal (GNPS) accessed via the following link: https://gnps.ucsd.edu/ProteoSAFe/index.jsp?params=\%7B\%22workflow\%22:\%22FEATUREBASED-MOLECULAR-

NETWORKING\%22,\%22library on server\%22:\%22d.speclibs;\%22\%7D.

The annotations obtained using this workflow fall under MSI level 2 or 3 and were used for feature analysis (Sumner et al., 2007). Briefly, level 2 compounds are putatively annotated meaning they are not identified using chemical reference standards but rather based on physical properties and/or spectral similarities to available spectral libraries (publicly available and purchased NIST17 CID). Level 3 compounds are putatively characterized classes of compounds identified similarly to level 2 compounds. The feature based molecular networking workflow on GNPS (Nothias et al., 2019) was utilized in order to analyze the spectra associated with the feature tables produced using the open source software Mzmine version 2.51 (Pluskal et al., 2010). The .mgf and .csv outputs from MZmine v2.51 were used to run the workflow. The GNPS workflow parameters used we as follows: Precursor lon Mass $0.02 \mathrm{Da}$, Fragment lon Mass Tolerance 0.02 Da, Min Pairs Cos 0.7, Minimum Matched Fragments 6, Maximum Shift Between Precursors 500 Da, Network TopK 10, Maximum Connected Component Size (Beta) 100 , and the files were row sum normalized. Default parameters were used for the rest of the 
settings. The visualizations and statistical analyses were performed using QIIME 2 v.2019.10 (Bolyen et al., 2019), MetaboAnalyst and Cytoscape v3.7.2. All PCoA axes in Figure 6 and Figure Supplement 5 were individually plotted in QIIME 2 and due to a batch effect between subsequent plates run on the mass spectrometer- axes that minimized experimental biases were determined and chosen for graphical representation. 


\section{REFERENCES}

Alavi, S., Mitchell, J.D., Cho, J.Y., Liu, R., Macbeth, J.C., and Hsiao, A. (2020). Interpersonal Gut Microbiome Variation Drives Susceptibility and Resistance to Cholera Infection. Cell 181, 1533-1546.e13.

Albenberg, L.G., and Wu, G.D. (2014). Diet and the intestinal microbiome: associations, functions, and implications for health and disease. Gastroenterology 146, 1564-1572.

Anders, S., Pyl, P.T., and Huber, W. (2015). HTSeq--a Python framework to work with highthroughput sequencing data. Bioinformatics 31, 166-169.

Anlauf, M., Schäfer, M.K.H., Eiden, L., and Weihe, E. (2003). Chemical coding of the human gastrointestinal nervous system: cholinergic, VIPergic, and catecholaminergic phenotypes. The Journal of Comparative Neurology 459, 90-111.

Aries, V., Crowther, J.S., Drasar, B.S., and Hill, M.J. (1969). Degradation of bile salts by human intestinal bacteria. Gut 10, 575-576.

Bahrami, S., and Drabløs, F. (2016). Gene regulation in the immediate-early response process. Advances in Biological Regulation 62, 37-49.

Begley, M., Sleator, R.D., Gahan, C.G.M., and Hill, C. (2005). Contribution of three bileassociated loci, bsh, pva, and btlB, to gastrointestinal persistence and bile tolerance of Listeria monocytogenes. Infect. Immun. 73, 894-904.

Benskey, M.J., Kuhn, N.C., Galligan, J.J., Garcia, J., Boye, S.E., Hauswirth, W.W., Mueller, C., Boye, S.L., and Manfredsson, F.P. (2015). Targeted gene delivery to the enteric nervous system using AAV: a comparison across serotypes and capsid mutants. Mol. Ther. 23, 488500 .

Bhavsar, A.S., Verma, S., Lamba, R., Lall, C.G., Koenigsknecht, V., and Rajesh, A. (2013). Abdominal manifestations of neurologic disorders. Radiographics 33, 135-153.

Bolyen, E., Rideout, J.R., Dillon, M.R., Bokulich, N.A., Abnet, C.C., Al-Ghalith, G.A., Alexander, H., Alm, E.J., Arumugam, M., Asnicar, F., et al. (2019). Reproducible, interactive, scalable and extensible microbiome data science using QIIME 2. Nat Biotechnol 37, 852-857.

Bravo, J.A., Forsythe, P., Chew, M.V., Escaravage, E., Savignac, H.M., Dinan, T.G., Bienenstock, J., and Cryan, J.F. (2011). Ingestion of Lactobacillus strain regulates emotional behavior and central GABA receptor expression in a mouse via the vagus nerve. Proceedings of the National Academy of Sciences 108, 16050-16055.

Bruel-Jungerman, E., Davis, S., and Laroche, S. (2016). Brain Plasticity Mechanisms and Memory: A Party of Four. Neuroscientist 13, 492-505.

Carabotti, M., Scirocco, A., Maselli, M.A., and Severi, C. (2015). The gut-brain axis: interactions between enteric microbiota, central and enteric nervous systems. Ann Gastroenterol 28, 203209. 
Carroll, I.M., Ringel-Kulka, T., Ferrier, L., Wu, M.C., Siddle, J.P., Bueno, L., and Ringel, Y. (2013). Fecal protease activity is associated with compositional alterations in the intestinal microbiota. PloS One 8, e78017.

Caspi, R., Billington, R., Fulcher, C.A., Keseler, I.M., Kothari, A., Krummenacker, M., Latendresse, M., Midford, P.E., Ong, Q., Ong, W.K., et al. (2018). The MetaCyc database of metabolic pathways and enzymes. Nucleic Acids Res 46, D633-D639.

Cekanaviciute, E., Yoo, B.B., Runia, T.F., Debelius, J.W., Singh, S., Nelson, C.A., Kanner, R., Bencosme, Y., Lee, Y.K., Hauser, S.L., et al. (2017). Gut bacteria from multiple sclerosis patients modulate human T cells and exacerbate symptoms in mouse models. Proc. Natl. Acad. Sci. U.S.a. 114, 10713-10718.

Cersosimo, M.G., Raina, G.B., Pecci, C., Pellene, A., Calandra, C.R., Gutiérrez, C., Micheli, F.E., and Benarroch, E.E. (2013). Gastrointestinal manifestations in Parkinson's disease: prevalence and occurrence before motor symptoms. J. Neurol. 260, 1332-1338.

Challis, R.C., Ravindra Kumar, S., Chan, K.Y., Challis, C., Beadle, K., Jang, M.J., Kim, H.M., Rajendran, P.S., Tompkins, J.D., Shivkumar, K., et al. (2019). Systemic AAV vectors for widespread and targeted gene delivery in rodents. Nature Protocols 14, 379-414.

Chan, K.Y., Jang, M.J., Yoo, B.B., Greenbaum, A., Ravi, N., Wu, W.-L., Sánchez-Guardado, L., Lois, C., Mazmanian, S.K., Deverman, B.E., et al. (2017). Engineered AAVs for efficient noninvasive gene delivery to the central and peripheral nervous systems. Nat. Neurosci. 20 , 1172-1179.

Chen, T.-W., Wardill, T.J., Sun, Y., Pulver, S.R., Renninger, S.L., Baohan, A., Schreiter, E.R., Kerr, R.A., Orger, M.B., Jayaraman, V., et al. (2013). Ultrasensitive fluorescent proteins for imaging neuronal activity. Nature 499, 295-300.

Cociorva, D., L Tabb, D., and Yates, J.R. (2007). Validation of Tandem Mass Spectrometry Database Search Results Using DTASelect. Current Protocols in Bioinformatics 16.

Costello, M., Fleharty, M., Abreu, J., Farjoun, Y., Ferriera, S., Holmes, L., Granger, B., Green, L., Howd, T., Mason, T., et al. (2018). Characterization and remediation of sample index swaps by non-redundant dual indexing on massively parallel sequencing platforms. BMC Genomics 19, 332-10.

de Aguiar Vallim, T.Q., Tarling, E.J., and Edwards, P.A. (2013). Pleiotropic roles of bile acids in metabolism. Cell Metabolism 17, 657-669.

Del Giudice, E., Staiano, A., Capano, G., Romano, A., Florimonte, L., Miele, E., Ciarla, C., Campanozzi, A., and Crisanti, A.F. (1999). Gastrointestinal manifestations in children with cerebral palsy. Brain Dev. 21, 307-311.

Delpino, M.V., Marchesini, M.I., Estein, S.M., Comerci, D.J., Cassataro, J., Fossati, C.A., and Baldi, P.C. (2007). A bile salt hydrolase of Brucella abortus contributes to the establishment of a successful infection through the oral route in mice. Infect. Immun. 75, 299-305.

Deneux, T., Kaszas, A., Szalay, G., Katona, G., Lakner, T., Grinvald, A., Rózsa, B., and Vanzetta, I. (2016). Accurate spike estimation from noisy calcium signals for ultrafast three- 
dimensional imaging of large neuronal populations in vivo. Nature Communications 7, 1219017.

Derrien, M., Vaughan, E.E., Plugge, C.M., and de Vos, W.M. (2004). Akkermansia muciniphila gen. nov., sp. nov., a human intestinal mucin-degrading bacterium. International Journal of Systematic and Evolutionary Microbiology 54, 1469-1476.

Deverman, B.E., Pravdo, P.L., Simpson, B.P., Kumar, S.R., Chan, K.Y., Banerjee, A., Wu, W.L., Yang, B., Huber, N., Pasca, S.P., et al. (2016). Cre-dependent selection yields AAV variants for widespread gene transfer to the adult brain. Nat Biotechnol 34, 204-209.

Didion, J.P., Martin, M., and Collins, F.S. (2017). Atropos: specific, sensitive, and speedy trimming of sequencing reads. PeerJ 5, e3720.

Donaldson, G.P., Ladinsky, M.S., Yu, K.B., Sanders, J.G., Yoo, B.B., Chou, W.C., Conner, M.E., Earl, A.M., Knight, R., Bjorkman, P.J., et al. (2018). Gut microbiota utilize immunoglobulin A for mucosal colonization. Science 360, 795-800.

Donaldson, G.P., Lee, S.M., and Mazmanian, S.K. (2015). Gut biogeography of the bacterial microbiota. Nat Rev Micro 14, 20-32.

Donowitz, M., Singh, S., Salahuddin, F.F., Hogema, B.M., Chen, Y., Gucek, M., Cole, R.N., Ham, A., Zachos, N.C., Kovbasnjuk, O., et al. (2007). Proteome of murine jejunal brush border membrane vesicles. J. Proteome Res. 6, 4068-4079.

Drokhlyansky, E., Smillie, C.S., Van Wittenberghe, N., Ericsson, M., Griffin, G.K., Eraslan, G., Dionne, D., Cuoco, M.S., Goder-Reiser, M.N., Sharova, T., et al. (2020). The Human and Mouse Enteric Nervous System at Single-Cell Resolution. Cell 182, 1606-1622.e1623.

Elias, J.E., and Gygi, S.P. (2007). Target-decoy search strategy for increased confidence in large-scale protein identifications by mass spectrometry. Nat. Methods 4, 207-214.

Everard, A., Belzer, C., Geurts, L., Ouwerkerk, J.P., Druart, C., Bindels, L.B., Guiot, Y., Derrien, M., Muccioli, G.G., Delzenne, N.M., et al. (2013). Cross-talk between Akkermansia muciniphila and intestinal epithelium controls diet-induced obesity. Proc. Natl. Acad. Sci. U.S.a. 110, 90669071.

Fiorucci, S., Biagioli, M., Zampella, A., and Distrutti, E. (2018). Bile Acids Activated Receptors Regulate Innate Immunity. Frontiers in Immunology 9, eaao1605-eaao1617.

Flandez, M., Guilmeau, S., Blache, P., and Augenlicht, L.H. (2008). KLF4 regulation in intestinal epithelial cell maturation. Experimental Cell Research 314, 3712-3723.

Foong, J.P.P., Tough, I.R., Cox, H.M., and Bornstein, J.C. (2014). Properties of cholinergic and non-cholinergic submucosal neurons along the mouse colon. The Journal of Physiology 592, 777-793.

Franzosa, E.A., Mclver, L.J., Rahnavard, G., Thompson, L.R., Schirmer, M., Weingart, G., Lipson, K.S., Knight, R., Caporaso, J.G., Segata, N., et al. (2018). Species-level functional profiling of metagenomes and metatranscriptomes. Nat. Methods 15, 962-968. 
Fried, D.E., and Gulbransen, B.D. (2015). In situ Ca2+ imaging of the enteric nervous system. JoVE.

Fu, L., Niu, B., Zhu, Z., Wu, S., and Li, W. (2012). CD-HIT: accelerated for clustering the nextgeneration sequencing data. Bioinformatics 28, 3150-3152.

Furness, J.B. (2012). The enteric nervous system and neurogastroenterology. Nature Reviews Gastroenterology and Hepatology 9, 286-294.

Furness, J.B., Callaghan, B.P., Rivera, L.R., and Cho, H.-J. (2014). The Enteric Nervous System and Gastrointestinal Innervation: Integrated Local and Central Control. In The Enteric Nervous System, (New York, NY: Springer New York), pp. 39-71.

Furness, J.B. (2006). The Enteric Nervous System (Wiley-Blackwell).

Gabanyi, I., Muller, P.A., Feighery, L., Oliveira, T.Y., Costa-Pinto, F.A., and Mucida, D. (2016). Neuro-immune Interactions Drive Tissue Programming in Intestinal Macrophages. Cell 164, 378-391.

Gatto, L., and Lilley, K.S. (2012). MSnbase-an R/Bioconductor package for isobaric tagged mass spectrometry data visualization, processing and quantitation. Bioinformatics 28, 288-289.

Gershon, M.D. (2015). The Enteric Nervous System: A Second Brain. Hospital Practice 34, 3152.

Glenn, T.C., Nilsen, R.A., Kieran, T.J., Sanders, J.G., Bayona-Vásquez, N.J., Finger, J.W., Pierson, T.W., Bentley, K.E., Hoffberg, S.L., Louha, S., et al. (2019). Adapterama I: universal stubs and primers for 384 unique dual-indexed or 147,456 combinatorially-indexed Illumina libraries (iTru \& iNext). PeerJ 7, e7755.

Gonzalez, A., Navas-Molina, J.A., Kosciolek, T., McDonald, D., Vázquez-Baeza, Y., Ackermann, G., DeReus, J., Janssen, S., Swafford, A.D., Orchanian, S.B., et al. (2018). Qiita: rapid, web-enabled microbiome meta-analysis. Nat. Methods 15, 796-798.

Gottlieb, A., and Canbay, A. (2019). Why Bile Acids Are So Important in Non-Alcoholic Fatty Liver Disease (NAFLD) Progression. Cells 8.

Grundy, D., and Brookes, S. (2011). Neural Control of Gastrointestinal Function. Colloquium Series on Integrated Systems Physiology: From Molecule to Function 3, 1-134.

Gurdeep Singh, R., Tanca, A., Palomba, A., Van der Jeugt, F., Verschaffelt, P., Uzzau, S., Martens, L., Dawyndt, P., and Mesuere, B. (2019). Unipept 4.0: Functional Analysis of Metaproteome Data. J. Proteome Res. 18, 606-615.

Haenraets, K., Foster, E., Johannssen, H., Kandra, V., Frezel, N., Steffen, T., Jaramillo, V., Paterna, J.-C., Zeilhofer, H.U., and Wildner, H. (2017). Spinal nociceptive circuit analysis with recombinant adeno-associated viruses: the impact of serotypes and promoters. Journal of Neurochemistry 142, 721-733. 
Hama, H., Hioki, H., Namiki, K., Hoshida, T., Kurokawa, H., Ishidate, F., Kaneko, T., Akagi, T., Saito, T., Saido, T., et al. (2015). ScaleS: an optical clearing palette for biological imaging. Nat. Neurosci. 18, 1518-1529.

Hansen, M.B. (2003). The enteric nervous system II: Gastrointestinal functions. Pharmacol. Toxicol. 92, 249-257.

He, L., Diedrich, J., Chu, Y.-Y., and Yates, J.R. (2015). Extracting Accurate Precursor Information for Tandem Mass Spectra by RawConverter. Anal. Chem. 87, 11361-11367.

Hennig, G.W., Gould, T.W., Koh, S.D., Corrigan, R.D., Heredia, D.J., Shonnard, M.C., and Smith, T.K. (2015). Use of Genetically Encoded Calcium Indicators (GECls) Combined with Advanced Motion Tracking Techniques to Examine the Behavior of Neurons and Glia in the Enteric Nervous System of the Intact Murine Colon. Front. Cell. Neurosci. 9, 13819.

Hillmann, B., Al-Ghalith, G.A., Shields-Cutler, R.R., Zhu, Q., Gohl, D.M., Beckman, K.B., Knight, R., and Knights, D. (2018). Evaluating the Information Content of Shallow Shotgun Metagenomics. mSystems 3, 457.

Hofmann, A.F., and Eckmann, L. (2006). How bile acids confer gut mucosal protection against bacteria. Proceedings of the National Academy of Sciences 103, 4333-4334.

Huber, W., Heydebreck, von, A., Sültmann, H., Poustka, A., and Vingron, M. (2002). Variance stabilization applied to microarray data calibration and to the quantification of differential expression. Bioinformatics 18 Suppl 1, S96-S104.

Hyland, N.P., and Cryan, J.F. (2010). A Gut Feeling about GABA: Focus on GABA(B) Receptors. Front Pharmacol 1, 124.

Ichikawa, R., Takayama, T., Yoneno, K., Kamada, N., Kitazume, M.T., Higuchi, H., Matsuoka, K., Watanabe, M., Itoh, H., Kanai, T., et al. (2012). Bile acids induce monocyte differentiation toward interleukin-12 hypo-producing dendritic cells via a TGR5-dependent pathway. Immunology 136, 153-162.

Jackson, K.L., Dayton, R.D., Deverman, B.E., and Klein, R.L. (2016). Better Targeting, Better Efficiency for Wide-Scale Neuronal Transduction with the Synapsin Promoter and AAV-PHP.B. Front. Mol. Neurosci. 9, 116.

Jangi, S., Gandhi, R., Cox, L.M., Li, N., Glehn, von, F., Yan, R., Patel, B., Mazzola, M.A., Liu, S., Glanz, B.L., et al. (2016). Alterations of the human gut microbiome in multiple sclerosis. Nature Communications 7, 12015-11.

Jarret, A., Jackson, R., Duizer, C., Healy, M.E., Zhao, J., Rone, J.M., Bielecki, P., Sefik, E., Roulis, M., Rice, T., et al. (2020). Enteric Nervous System-Derived IL-18 Orchestrates Mucosal Barrier Immunity. Cell 180, 50-63.e12.

Jia, L., Betters, J.L., and Yu, L. (2011). Niemann-pick C1-like 1 (NPC1L1) protein in intestinal and hepatic cholesterol transport. Annu. Rev. Physiol. 73, 239-259. 
Johnson, C.D., Barlow-Anacker, A.J., Pierre, J.F., Touw, K., Erickson, C.S., Furness, J.B., Epstein, M.L., and Gosain, A. (2018). Deletion of choline acetyltransferasein enteric neurons results in postnatal intestinal dysmotility and dysbiosis. The FASEB Journal 32, 4744-4752.

Jones, B.V., Begley, M., Hill, C., Gahan, C.G.M., and Marchesi, J.R. (2008). Functional and comparative metagenomic analysis of bile salt hydrolase activity in the human gut microbiome. Proc. Natl. Acad. Sci. U.S.a. 105, 13580-13585.

Kaelberer, M.M., Buchanan, K.L., Klein, M.E., Barth, B.B., Montoya, M.M., Shen, X., and Bohórquez, D.V. (2018). A gut-brain neural circuit for nutrient sensory transduction. Science 361 , eaat5236.

Kim, D., Langmead, B., and Salzberg, S.L. (2015). HISAT: a fast spliced aligner with low memory requirements. Nat. Methods 12, 357-360.

Kirwan, W.O., Smith, A.N., Mitchell, W.D., Falconer, J.D., and Eastwood, M.A. (1975). Bile acids and colonic motility in the rabbit and the human. Gut 16, 894-902.

Kotterman, M.A., and Schaffer, D.V. (2014). Engineering adeno-associated viruses for clinical gene therapy. Nat Rev Genet 15, 445-451.

Kügler, S., Kilic, E., and Bähr, M. (2003). Human synapsin 1 gene promoter confers highly neuron-specific long-term transgene expression from an adenoviral vector in the adult rat brain depending on the transduced area. Gene Ther 10, 337-347.

Lai, N.Y., Musser, M.A., Pinho-Ribeiro, F.A., Baral, P., Jacobson, A., Ma, P., Potts, D.E., Chen, Z., Paik, D., Soualhi, S., et al. (2019). Gut-Innervating Nociceptor Neurons Regulate Peyer's Patch Microfold Cells and SFB Levels to Mediate Salmonella Host Defense. Cell 180, 3349.e22.

Langmead, B., and Salzberg, S.L. (2012). Fast gapped-read alignment with Bowtie 2. Nat. Methods 9, 357-359.

Latgé, J.P. (2007). The cell wall: a carbohydrate armour for the fungal cell. Molecular Microbiology 66, 279-290.

Li, W., and Godzik, A. (2006). Cd-hit: a fast program for clustering and comparing large sets of protein or nucleotide sequences. Bioinformatics 22, 1658-1659.

Li, W., Jaroszewski, L., and Godzik, A. (2001). Clustering of highly homologous sequences to reduce the size of large protein databases. Bioinformatics 17, 282-283.

Li, Z., Chalazonitis, A., Huang, Y.Y., Mann, J.J., Margolis, K.G., Yang, Q.M., Kim, D.O., Cote, F., Mallet, J., and Gershon, M.D. (2011). Essential Roles of Enteric Neuronal Serotonin in Gastrointestinal Motility and the Development/Survival of Enteric Dopaminergic Neurons. Journal of Neuroscience 31, 8998-9009.

Lindeberg, J., Usoskin, D., Bengtsson, H., Gustafsson, A., Kylberg, A., Söderström, S., and Ebendal, T. (2004). Transgenic expression of Cre recombinase from the tyrosine hydroxylase locus. Genesis 40, 67-73. 
Long, S.L., Gahan, C.G.M., and Joyce, S.A. (2017). Interactions between gut bacteria and bile in health and disease. Mol. Aspects Med. 56, 54-65.

Love, M.I., Huber, W., and Anders, S. (2014). Moderated estimation of fold change and dispersion for RNA-seq data with DESeq2. Genome Biol 15, 1-21.

Martino, C., Morton, J.T., Marotz, C.A., Thompson, L.R., Tripathi, A., Knight, R., and Zengler, K. (2019). A Novel Sparse Compositional Technique Reveals Microbial Perturbations. mSystems $4,813$.

Matheis, F., Muller, P.A., Graves, C.L., Gabanyi, I., Kerner, Z.J., Costa-Borges, D., Ahrends, T., Rosenstiel, P., and Mucida, D. (2020). Adrenergic Signaling in Muscularis Macrophages Limits Infection-Induced Neuronal Loss. Cell 180,64-78.e16.

Matsushima, S., Hori, S., and Matsuda, M. (1986). Conversion of 4-aminobutyraldehyde to gamma-aminobutyric acid in striatum treated with semicarbazide and kainic acid. Neurochem. Res. 11, 1313-1319.

McConnell, R.E., Benesh, A.E., Mao, S., Tabb, D.L., and Tyska, M.J. (2011). Proteomic analysis of the enterocyte brush border. American Journal of Physiology - Gastrointestinal and Liver Physiology 300, G914-G926.

McMillin, M., and DeMorrow, S. (2016). Effects of bile acids on neurological function and disease. Faseb J. 30, 3658-3668.

Mesuere, B., Debyser, G., Aerts, M., Devreese, B., Vandamme, P., and Dawyndt, P. (2015). The Unipept metaproteomics analysis pipeline. Proteomics 15, 1437-1442.

Miano, J.M., Miano, J.M., Vlasic, N., Vlasic, N., TOTA, R.R., Tota, R.R., STEMERMAN, M.B., and Stemerman, M.B. (1993). Smooth muscle cell immediate-early gene and growth factor activation follows vascular injury. A putative in vivo mechanism for autocrine growth. Arterioscler. Thromb. 13, 211-219.

Mittal, R., Debs, L.H., Patel, A.P., Nguyen, D., Patel, K., O'Connor, G., Grati, M., Mittal, J., Yan, D., Eshraghi, A.A., et al. (2017). Neurotransmitters: The Critical Modulators Regulating GutBrain Axis. J. Cell. Physiol. 232, 2359-2372.

Muller, P.A., Koscsó, B., Rajani, G.M., Stevanovic, K., Berres, M.-L., Hashimoto, D., Mortha, A., Leboeuf, M., Li, X.-M., Mucida, D., et al. (2014). Crosstalk between Muscularis Macrophages and Enteric Neurons Regulates Gastrointestinal Motility. Cell 158, 1210.

Nishiyama, H., Nagai, T., Kudo, M., Okazaki, Y., Azuma, Y., Watanabe, T., Goto, S., Ogata, H., and Sakurai, T. (2018). Supplementation of pancreatic digestive enzymes alters the composition of intestinal microbiota in mice. Biochem. Biophys. Res. Commun. 495, 273-279.

Nothias, L.F., Petras, D., Schmid, R., Dührkop, K., Rainer, J., Sarvepalli, A., Protsyuk, I., Ernst, M., Tsugawa, H., Fleischauer, M., et al. (2019). Feature-based Molecular Networking in the GNPS Analysis Environment. bioRxiv 38, 812404. 
Olson, C.A., Vuong, H.E., Yano, J.M., Liang, Q.Y., Nusbaum, D.J., and Hsiao, E.Y. (2018). The Gut Microbiota Mediates the Anti-Seizure Effects of the Ketogenic Diet. Cell 173, 17281741.e13.

Park, S.K.R., Jung, T., Thuy-Boun, P.S., Wang, A.Y., John R Yates, I., and Wolan, D.W. (2018). ComPIL 2.0: An Updated Comprehensive Metaproteomics Database. J. Proteome Res. 18, 616-622.

Park, S.K., Venable, J.D., Xu, T., and Yates, J.R. (2008). A quantitative analysis software tool for mass spectrometry-based proteomics. Nat. Methods 5, 319-322.

Peng, J., Elias, J.E., Thoreen, C.C., Licklider, L.J., and Gygi, S.P. (2003). Evaluation of multidimensional chromatography coupled with tandem mass spectrometry (LC/LC-MS/MS) for large-scale protein analysis: the yeast proteome. J. Proteome Res. 2, 43-50.

Pfeiffer, R.F. (2003). Gastrointestinal dysfunction in Parkinson's disease. Lancet Neurol 2, 107116.

Plovier, H., Everard, A., Druart, C., Depommier, C., Van Hul, M., Geurts, L., Chilloux, J., Ottman, N., Duparc, T., Lichtenstein, L., et al. (2017). A purified membrane protein from Akkermansia muciniphila or the pasteurized bacterium improves metabolism in obese and diabetic mice. Nature Medicine 23, 107-113.

Pluskal, T., Castillo, S., Villar-Briones, A., and Oresic, M. (2010). MZmine 2: Modular framework for processing, visualizing, and analyzing mass spectrometry-based molecular profile data. BMC Bioinformatics 11.

Rakhilin, N., Barth, B., Choi, J., Muñoz, N.L., Kulkarni, S., Jones, J.S., Small, D.M., Cheng, Y.T., Cao, Y., LaVinka, C., et al. (2016). Simultaneous optical and electrical in vivo analysis of the enteric nervous system. Nature Communications 7,31 .

Rakhilin, N., Garrett, A., Eom, C.-Y., Chavez, K.R., Small, D.M., Daniel, A.R., Kaelberer, M.M., Mejooli, M.A., Huang, Q., Ding, S., et al. (2019). An intravital window to image the colon in real time. Nature Communications 10, 5647-10.

Ramirez-Carrozzi, V.R., Braas, D., Bhatt, D.M., Cheng, C.S., Hong, C., Doty, K.R., Black, J.C., Hoffmann, A., Carey, M., and Smale, S.T. (2009). A Unifying Model for the Selective Regulation of Inducible Transcription by CpG Islands and Nucleosome Remodeling. Cell 138, 114-128.

Rao, M., and Gershon, M.D. (2016). The bowel and beyond: the enteric nervous system in neurological disorders. Nature Reviews Gastroenterology and Hepatology 13, 517-528.

Ritchie, M.E., Phipson, B., Wu, D., Hu, Y., Law, C.W., Shi, W., and Smyth, G.K. (2015). limma powers differential expression analyses for RNA-sequencing and microarray studies. Nucleic Acids Res 43, e47-e47.

Rodríguez-Piñeiro, A.M., Bergström, J.H., Ermund, A., Gustafsson, J.K., Schütte, A., Johansson, M.E.V., and Hansson, G.C. (2013). Studies of mucus in mouse stomach, small intestine, and colon. II. Gastrointestinal mucus proteome reveals Muc2 and Muc5ac accompanied by a set of core proteins. American Journal of Physiology - Gastrointestinal and Liver Physiology 305, G348-G356. 
Sakai, K., Makino, T., Kawai, Y., and Mutai, M. (1980). Intestinal microflora and bile acids. Effect of bile acids on the distribution of microflora and bile acid in the digestive tract of the rat. Microbiol. Immunol. 24, 187-196.

Samulski, R.J., and Muzyczka, N. (2014). AAV-Mediated Gene Therapy for Research and Therapeutic Purposes. Annu Rev Virol 1, 427-451.

Sang, Q., and Young, H.M. (1998). The identification and chemical coding of cholinergic neurons in the small and large intestine of the mouse. Anat. Rec. 251, 185-199.

Sannasiddappa, T.H., Lund, P.A., and Clarke, S.R. (2017). In Vitro Antibacterial Activity of Unconjugated and Conjugated Bile Salts on Staphylococcus aureus. Front. Microbio. 8, 1581.

Schneider, S., Wright, C.M., and Heuckeroth, R.O. (2019). Unexpected Roles for the Second Brain: Enteric Nervous System as Master Regulator of Bowel Function. Annu. Rev. Physiol. 81, 235-259.

Segata, N., Izard, J., Waldron, L., Gevers, D., Miropolsky, L., Garrett, W.S., and Huttenhower, C. (2011). Metagenomic biomarker discovery and explanation. Genome Biol 12, R60.

Seillet, C., Luong, K., Tellier, J., Jacquelot, N., Shen, R.D., Hickey, P., Wimmer, V.C., Whitehead, L., Rogers, K., Smyth, G.K., et al. (2020). The neuropeptide VIP confers anticipatory mucosal immunity by regulating ILC3 activity. Nat Immunol 21, 168-177.

Sinha, R., Stanley, G., Gulati, G.S., Ezran, C., Travaglini, K.J., Wei, E., Chan, C.K.F., Nabhan, A.N., Su, T., Morganti, R.M., et al. (2017). Index switching causes "spreading-of-signal" among multiplexed samples in Illumina HiSeq 4000 DNA sequencing. 29, 1072-29.

Steele, P.A., Brookes, S.J., and Costa, M. (1991). Immunohistochemical identification of cholinergic neurons in the myenteric plexus of guinea-pig small intestine. Neuroscience 45 , 227-239.

Sumner, L.W., Amberg, A., Barrett, D., Beale, M.H., Beger, R., Daykin, C.A., Fan, T.W.M., Fiehn, O., Goodacre, R., Griffin, J.L., et al. (2007). Proposed minimum reporting standards for chemical analysis Chemical Analysis Working Group (CAWG) Metabolomics Standards Initiative (MSI). Metabolomics 3, 211-221.

Sundararajan, Z., Knoll, R., Hombach, P., Becker, M., Schultze, J.L., and Ulas, T. (2019). ShinySeq: advanced guided transcriptome analysis. BMC Res Notes 12, 432-435.

Suzek, B.E., Huang, H., McGarvey, P., Mazumder, R., and Wu, C.H. (2007). UniRef: comprehensive and non-redundant UniProt reference clusters. Bioinformatics 23, 1282-1288.

Szklarczyk, D., Gable, A.L., Lyon, D., Junge, A., Wyder, S., Huerta-Cepas, J., Simonovic, M., Doncheva, N.T., Morris, J.H., Bork, P., et al. (2019). STRING v11: protein-protein association networks with increased coverage, supporting functional discovery in genome-wide experimental datasets. Nucleic Acids Res 47, D607-D613.

Tabb, D.L., McDonald, W.H., and Yates, J.R. (2002). DTASelect and Contrast: tools for assembling and comparing protein identifications from shotgun proteomics. J. Proteome Res. 1, 21-26. 
Talbot, J., Hahn, P., Kroehling, L., Nguyen, H., Li, D., and Littman, D.R. (2020). Feedingdependent VIP neuron-ILC3 circuit regulates the intestinal barrier. Nature 579, 575-580.

Thompson, K.J., Khajehali, E., Bradley, S.J., Navarrete, J.S., Huang, X.P., Slocum, S., Jin, J., Liu, J., Xiong, Y., Olsen, R.H.J., et al. (2018). DREADD Agonist 21 Is an Effective Agonist for Muscarinic-Based DREADDs in Vitro and in Vivo. ACS Pharmacol Transl Sci 1, 61-72.

Treweek, J.B., Chan, K.Y., Flytzanis, N.C., Yang, B., Deverman, B.E., Greenbaum, A., Lignell, A., Xiao, C., Cai, L., Ladinsky, M.S., et al. (2015). Whole-body tissue stabilization and selective extractions via tissue-hydrogel hybrids for high-resolution intact circuit mapping and phenotyping. Nature Protocols 10, 1860-1896.

Truong, D.T., Franzosa, E.A., Tickle, T.L., Scholz, M., Weingart, G., Pasolli, E., Tett, A., Huttenhower, C., and Segata, N. (2015). MetaPhIAn2 for enhanced metagenomic taxonomic profiling. Nat. Methods 12, 902-903.

Valicenti-McDermott, M.D., McVicar, K., Cohen, H.J., Wershil, B.K., and Shinnar, S. (2008). Gastrointestinal symptoms in children with an autism spectrum disorder and language regression. Pediatr. Neurol. 39, 392-398.

Van Herreweghen, F., Van den Abbeele, P., De Mulder, T., De Weirdt, R., Geirnaert, A., Hernandez-Sanabria, E., Vilchez-Vargas, R., Jauregui, R., Pieper, D.H., Belzer, C., et al. (2017). In vitro colonisation of the distal colon by Akkermansia muciniphila is largely mucin and $\mathrm{pH}$ dependent. Benef Microbes 8, 81-96.

Vavassori, P., Mencarelli, A., Renga, B., Distrutti, E., and Fiorucci, S. (2009). The bile acid receptor $\mathrm{FXR}$ is a modulator of intestinal innate immunity. J Immunol 183, 6251-6261.

Walsh, K.T., and Zemper, A.E. (2019). The Enteric Nervous System for Epithelial Researchers: Basic Anatomy, Techniques, and Interactions With the Epithelium. Cellular and Molecular Gastroenterology and Hepatology 8, 369-378.

Wang, M., Carver, J.J., Phelan, V.V., Sanchez, L.M., Garg, N., Peng, Y., Nguyen, D.D., Watrous, J., Kapono, C.A., Luzzatto-Knaan, T., et al. (2016). Sharing and community curation of mass spectrometry data with Global Natural Products Social Molecular Networking. Nat Biotechnol 34, 828-837.

Watanabe, M., Houten, S.M., Mataki, C., Christoffolete, M.A., Kim, B.W., Sato, H., Messaddeq, N., Harney, J.W., Ezaki, O., Kodama, T., et al. (2006). Bile acids induce energy expenditure by promoting intracellular thyroid hormone activation. Nature 439, 484-489.

Wess, J., Nakajima, K., and Jain, S. (2013). Novel designer receptors to probe GPCR signaling and physiology. Trends Pharmacol. Sci. 34, 385-392.

Wessel, D., and Flügge, U.I. (1984). A method for the quantitative recovery of protein in dilute solution in the presence of detergents and lipids. Analytical Biochemistry 138, 141-143.

Whitcomb, D.C., and Lowe, M.E. (2007). Human pancreatic digestive enzymes. Dig. Dis. Sci. $52,1-17$. 
Wu, Y.E., Pan, L., Zuo, Y., Li, X., and Hong, W. (2017). Detecting Activated Cell Populations Using Single-Cell RNA-Seq. Neuron 96, 313-329.e316.

Xu, T., Park, S.K., Venable, J.D., Wohlschlegel, J.A., Diedrich, J.K., Cociorva, D., Lu, B., Liao, L., Hewel, J., Han, X., et al. (2015). ProLuCID: An improved SEQUEST-like algorithm with enhanced sensitivity and specificity. J Proteomics 129, 16-24.

Xu, T., Venable, J.D., Park, S.K., Cociorva, D., Lu, Q.B., Liao, L., Wohlschlegel, J., Hewel, J., Yates, J.R., and Conciorva, D. (2006). ProLuCID, a fast and sensitive tandem mass spectrabased protein identification program.

Yang, B., Treweek, J.B., Kulkarni, R.P., Deverman, B.E., Chen, C.-K., Lubeck, E., Shah, S., Cai, L., and Gradinaru, V. (2014). Single-Cell Phenotyping within Transparent Intact Tissue through Whole-Body Clearing. Cell 158, 945-958.

Yano, J.M., Yu, K., Donaldson, G.P., Shastri, G.G., Ann, P., Ma, L., Nagler, C.R., Ismagilov, R.F., Mazmanian, S.K., and Hsiao, E.Y. (2015). Indigenous Bacteria from the Gut Microbiota Regulate Host Serotonin Biosynthesis. Cell 161, 264-276.

Zhang, H., Sparks, J.B., Karyala, S.V., Settlage, R., and Luo, X.M. (2015). Host adaptive immunity alters gut microbiota. Isme J 9, 770-781.

Zhang, X., Smits, A.H., van Tilburg, G.B., Ovaa, H., Huber, W., and Vermeulen, M. (2018). Proteome-wide identification of ubiquitin interactions using UbIA-MS. Nature Protocols 13, 530550.

Zhu, Q., Mai, U., Pfeiffer, W., Janssen, S., Asnicar, F., Sanders, J.G., Belda-Ferre, P., AlGhalith, G.A., Kopylova, E., McDonald, D., et al. (2019). Phylogenomics of 10,575 genomes reveals evolutionary proximity between domains Bacteria and Archaea. Nature Communications $10,5477-14$. 